\title{
Biological Organization Principles: Biogenesis, Cognition and Evolution of Living Systems ${ }^{\star}$
}

\author{
Carlos Maureira* \\ Neurobiology and Biology of Cognition Laboratory, Faculty of Sciences, University of Chile, Santiago, Chile \\ José García ${ }^{1}$ \\ Pontificia Universidad Católica de Valparaíso, 2362807 Valparaíso, Chile
}

\begin{abstract}
The Autopoiesis and Cognition Theory (ACT), by Maturana and Varela, based on the notions of Biological Closure and Structural Coupling, is a well-known theory on how to understand biological organization 1, 2, 3. Although, for example, the Free Energy Principle framework evokes some entailments of autopoiesis in a more formal setting [4, 5]; and ACT has been used in many fields, its impact has been restricted because it lacks quantitative analysis. Here we present a theoretical framework grounded in accepted and well-developed ideas from Mathematics and Physics which advance the understanding of the Principles of Biological Organization under the guidance of Biological Closure and Structural Coupling. The disciplines of Differential Geometry/Topology, Mechanics and Complex Dynamical Systems provide a powerful, elegant, and well-established body of knowledge to support our Biological Organization Principles (BOP) framework. In particular, Stochastic Mechanics and KAM theory (from Kolmogorov, Arnold and Moser theorem) allow us to develop, using the notions of Biological Closure and Structural Coupling, a central core of BOP termed Dynamical Closure Mechanism. Under the proposed framework, a wide variety of biological phenomena can be understood, shedding new light on biological explanations. However, an understanding of biological organization may require the re-evaluation of dogmas on how we think on biology as it seems inescapable that what is needed is an integration of analysis and notions derived from mathematics, physics, and biology to generate a new landscape of ideas.
\end{abstract}

\footnotetext{
${ }^{\star}$ Part of this work was presented in Neuroscience 2018 Anual Meeting: poster JJJ45

* Correspondence to

Email address: carlos.maureira@uchile.cl (Carlos Maureira)

${ }^{1}$ supported by the Grant CONICYT/FONDECYT/INICIACION/11180056.
} 
Keywords: Biological Organization Principles, Dynamical Systems, Bayesian Inference and Brian, FEP, Autopoiesis, Evolution, Cognition, Niche, KAM-dynamics

\section{Contents}

1 Introduction 4

$1.1 \quad$ Biological Closure and Structural Coupling $\ldots \ldots \ldots \ldots \ldots$. . . . . . . . . . . 5

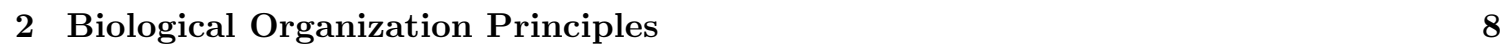

$2.1 \quad$ Mathematical and Physical Preamble. . . . . . . . . . . . . . . . . 11

$2.1 .1 \quad$ Differential Geometry and Topology $\ldots \ldots \ldots \ldots$. . . . . . . . . 11

$2.1 .2 \quad$ Components, Systems and Dynamics . . . . . . . . . . . . . . . 12

$2.1 .3 \quad$ Extremum Principles, Actions and Hamiltonians . . . . . . . . . . . . . 14

2.1 .4 Deterministic Chaos and Chaotic Behaviour . . . . . . . . . . . . . . . . 17

$2.2 \quad$ Organizing Chaos via Dynamical Closure and Structural Coupling . . . . . . . . . . 18

$2.2 .1 \quad$ Biological Organization . . . . . . . . . . . . . . . . . . . 19

2.3 The Dynamical Closure Mechanism, DCM . . . . . . . . . . . . . . . . . . 20

$2.3 .1 \quad$ Microscopic and Macroscopic DCM . . . . . . . . . . . . . . . . . . 22

$2.3 .2 \quad$ Biological Organization Space $\ldots \ldots \ldots \ldots \ldots$. . . . . . . . . . . 23

$2.3 .3 \quad$ Stochastic Mechanics and Probability of Functional Symmetries. . . . . . . . 24

2.4 Geometry and BOP Dynamics $\ldots \ldots \ldots \ldots \ldots \ldots \ldots$

$2.4 .1 \quad$ Dynamics and Flows in the DCM-Manifolds . . . . . . . . . . . . . . . . . 30

$2.4 .2 \quad$ Cognitive and Environmental Relations in BOP . . . . . . . . . . . . . . . 32

$2.4 .3 \quad$ Origin and Evolution from BOP $\ldots \ldots \ldots \ldots$. . . . . . . . . . 39

3 Discussion and Conclusions

$3.1 \quad$ Theoretical Key Ideas from BOP $\ldots \ldots \ldots \ldots$. . . . . . . . . . . . . . 43

$3.1 .1 \quad$ KAM-theoretical Dynamics $\ldots \ldots \ldots \ldots \ldots$. . . . . . . . . . . 43

$3.1 .2 \quad$ Flux Trajectories $\ldots \ldots \ldots \ldots \ldots$. . . . . . . . . . . . . 47

$3.1 .3 \quad$ Biological Organization Realm . . . . . . . . . . . . . . . . . . . . . . . . 49

3.1.4 Metaplastic Hamiltonian \& Diffusive Organization . . . . . . . . . . . . . . . 51

$3.1 .5 \quad$ Biological Organization a KAM-dynamical attractor $\ldots \ldots \ldots \ldots \ldots$ 


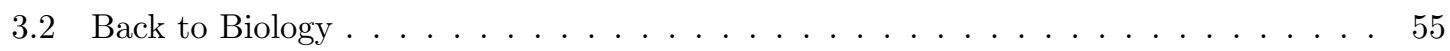

3.2 .1 BOP and FEP . . . . . . . . . . . . . . . . . . . . . . . . 55

3.2.2 BOP and Biogenesis \& Evolution . . . . . . . . . . . . . . . . . . . . . . . . . 60

3.2.3 Ontogeny and Phylogeny: Biological Structure of Space-Time . . . . . . . . . 62

3.2 .4 Variational Structure and Function . . . . . . . . . . . . . . . . . . . . . 6 63

$3.2 .5 \quad$ Functional Symmetries . . . . . . . . . . . . . . . . . . . . . . 64

3.2.6 Cognition as construction . . . . . . . . . . . . . . . . . . . . 66

$3.2 .7 \quad$ BOP's Mathematical-Physics Novelties. . . . . . . . . . . . . . . . . . . . . 67

3.2.8 Biological Organization as Mechanical Trajectories . . . . . . . . . . . . . . . 68

3.2.9 Complexification of the Biological Organization by a Simple Common Core, $\mathrm{DCM} \ldots \ldots \ldots \ldots 8$

3.2 .10 Inclusion of Other Theoretical Frameworks ． . . . . . . . . . . . . . . . . . . 69

3.2.11 Reevaluation of Autopoiesis and Cognition Theory and perspectives . . . . . 69

3.3 Final Summary . . . . . . . . . . . . . . . . . . . . . . . . . . . . 71

3.3.1 homine digitus . . . . . . . . . . . . . . . . . . . . . . . 74 


\section{Introduction}

Understanding Biology in a mathematically formal manner, as we understand inanimate physical nature has been a persisting yet unattainable goal in modern science [6, 7, 8. Modern Biology, although it is a sophisticated experimental science, still suffers from uncertain theoretical underpinning: 2 . Thus current biological principles, although supported by a wide spectrum of ideas ranging from physical-chemistry to evolutionary theory, is in search of its own formal logic [8, 9, 10, 11. Whether such a biological formal framework exists has divided scientists and philosophers. Immanuel Kant thought a "Newton of a blade of grass" would not exist because biology exhibits a natural purpose contrary to the direct causality found in the inanimate matter [12, 13, 14, 15]. The teleological consistency found in biology appears to be a major impediment to revealing causality, given that, at least according to Kant the causal agents in physics are the laws of nature [16, 17, 18.

Then, practitioners of the reductionist tradition assumed that an accumulation of experimental details would reveal the laws of a theory of biology. Thus, modern Cell Theory introduces the laws of physico-chemical mechanisms as the fundamental causal elements in cellular dynamics and living systems. Although this basic result and the continuous and intense effort, spanning many decades and subfields of biology, which has been successful in unraveling biological details, it has not led to a shared and accepted conceptual framework explaining the phenomenon of BIOLOGICAL Organization as an integrated totality. For example, for the eubacterium E. coli, containing about 5000 genes, 1200 metabolites, 1600 metabolic reactions, many signaling processes and replicating every 30 minutes, we do not match those details to explain how its metabolic network acts as an organized and autonomous wholeness. This long experimental pursuit in Biology has differed from the progress made in Physics in which the fundamental properties and laws of matter, and their associated dynamics, has been very successful starting from theoretical principles and using experiments to test basic theoretical principles [19, 20], see also [21].

Here we present a framework to understand BIOLOGICAL ORGANizATION integrating biological notions such as behavior, plasticity, cognition, niche, and the environment with notions taken from Differential Geometry, Dynamical Systems, and Mathematical-Physics. We use mechanical concepts to introduce a central core of biological organization, termed Dynamical Closure Mechanism (DCM), which orchestrates Biological Closure and Structural Coupling (BC\&SC) into a

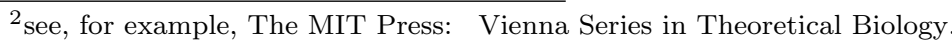


nonlinear dynamical setting. These two ideas in theoretical biology entail, self-construction, and self-organization (Biological Closure); and their ecological cognition associated with cognitive selfreference (Structural Coupling). On these grounds, we propose to understand the dynamics of the living organization by using stochastic and deterministic frameworks already developed to study mechanics. The continuous operation of the Dynamical Closure Mechanism (DCM) allows living systems to actively form Hamiltonian Organizational Dynamics which interact with the system's medium constrained by KAM-theory (from the Kolmogorov-Arnold-Moser theorem; 22, 23, 24]). Hamiltonian organizational dynamics denotes the capability of living systems to implement Hamiltonian dynamics in their organization. Notably, we claim that constrained by the KAM theorem, the recurrent system-medium interactions become incorporated into organizational dynamics. KAM theorem constraints allow the extraction of regularities to lie in quasi-integrable trajectories of the system organization: a key process defining the duality system-niche with cognitive and adaptive capabilities. The complementary approaches of stochastic and deterministic frameworks are crucial because they provide tools and concepts for the construction of DCM. It should be noted that DCM provides the mechanism underlying Structural Coupling and Biological Closure, the fundamental biological notions generating cognition, evolution, and behavior as well as biogenesis in light of Autopoiesis and Cognition Theory (ACT; $[1,2,3])$.

\subsection{Biological Closure and Structural Coupling}

Modern theories about biological organization rely on notions that are neither intuitive nor easy to understand. One of such ideas is Biological Closure, which has several emphasis depending on the contexts. We briefly mentioned them in previous sections but we will now focus on those that are most relevant to our proposal: topological circularity, self-fabrication, self-reference, self-organization, and Dynamical Closure. Topological circularity refers to the architecture of connectivity and disposition of parts and processes displaying a circular arrangement of connectivity. Topological circularity can be inferred even when many components and process are not fully determined. For example, diffuse networks with other topologies (local, small-world, etc) may reach circular topology in some network motifs or sub-networks. Self-fabrication is a second emphasis showing that living systems obviously posses because they produce almost all of their components using the very same network of processes and components that constitute them. The classical example is the metabolism in which metabolites and macro-molecules alike fabricate the macro- 
molecular components and processes constituting the metabolic network. Similarly, self-reference arises in relation to how systems apparently isolated from their environment coherently act on it. Self-reference is indicated then, because living systems act recursively on the results of their previous actions, such as the capacity for coherent operational changes accordingly to the overall circumstances. This action could refer to metabolism, the nervous system, or behavior. A fourth example, very well studied in nonlinear dynamics, is system self-organization, in which the focus is on the emergent properties that allow systems to reach higher levels of order (organization) from the interacting components and dynamics. A final facet is a Dynamical Closure, which appears in the context of analyzing the fluxes of actions and dynamical changes of the states of systems. In dynamical closure, the recurrence of activity fluxes and signals re-entrance defines crucial system dynamics while participating in systems control and error prediction as well as defining the architectural topology.

Although we have defined explicitly the above extensions of the Biological Closure concept, several mixed and overlapping uses can be found in the literature. An interesting, epistemological or philosophical use, it is found in the causality relations. Self-organization, Self-reference, and Self-fabrication have also been interpreted as examples of circular causality related to the idea of closure to efficient causes [25]. Perhaps the prime examples addressing Biological Closure appeared in biological literature when the need for a science of organization became apparent between 1930 and 1950 [26, 27, 8]. One of the first uses of closure refers to feedback introduced by Harold Black (1928) in the context of amplifier output control. In that context, feedback creates stability, a central property of biological regulation but it has not served to understand the stability of complex real networks. Later Norbert Wiener, in his influential book Cybernetics or Control and Communication in the Animal and the Machine [28], suggested that dense networks of feedback loops would provide stability for complex systems like metabolism and the brain. Biological Closure, with all its rich conceptual background, is a fundamental part of our proposal. Notably, the very concept of enzymes entails the notion of Biological Closure, either as in the metabolic concept or its functional architecture. We will use this entailment to introduce an important concept, the functional symmetries, in section 7 . Biological Closure, in conjunction with Structural Coupling, will be the central elements in our mechanistic core for Biological Organization (section 2.2.1).

As already mentioned, Biological Closure is also related to the dynamics of systems. Although it has been less explored so far, it can be characterized by many different conditions in the context 
of nonlinear dynamical systems. One of the simplest examples comes from looking at the action potential (AP) in the excitable tissue. In the APs, Biological Closure entails a dynamical aspect in which a systemic variable (i.e. a variable important to the organization like cell membrane potential $\left.\left(V_{m}\right)\right)$ is under functional control [29. In the case of $V_{m}$, its moment by moment value is controlled by metabolic processes that themselves are a function of $V_{m}$. In effect, the turnover number of the $\mathrm{Na} / \mathrm{K}$ pump and functional activity of ion channels depends on the dynamical construction of $V_{m}$. Crucially $V_{m}$, a systemic variable that cannot be ascribed to a single component or process, is a function that influences many processes that ultimately affects the very bio-synthetic pathway of $V_{m}$ regulation. Moreover, it is in the dynamical context that Biological Closure displays an important elaboration on this notion that has not been addressed by any other approaches [29]. In effect, Dynamical Closure provides a route for building a differential-geometry and mathematical-physics approach to Biological Organization, by introducing fluxes, gradients, divergent, and physical laws in a topological and geometrical setting. It is in the nervous system functions seen as excitable properties that neurons, synapses, and networks (i.e, different organizational levels) become embedded in manifolds and attractors of activity.

In the ACT, Structural Coupling uses the background notions of structure and organization; while distinguishes the structure as the components of the system; the organization relates to the configuration of relations between components. The structure of a system changes accordingly to the dynamics of internal interactions of components as well as because of the successive interactions with the medium. Systems and medium are structurally determined; consequently, the structure of a living system and the structure of the medium change together congruently, while their interactions are not destructive. The resulting history of two or more structure-determined systems becomes a history of spontaneous recursive structural changes, where all interacting systems change congruently until they diverge or disintegrate $[1,3,30$. This congruent dynamical change involving the structural changes emerging from the recurrent interaction of structure-determined systems is called structural coupling [30]. The structural determination of systems and environment and its structural coupling leads to the notion of constructivist epistemology. The consequences of structural coupling and its philosophical, cognitive, linguistic, and social sciences are beyond the present document. However, structural coupling in structure determined systems is intimately related to cognition and self-consciousness [30]. In this sense, cognition is the capacity that living systems to operate in dynamical congruence with the medium in which exists. It is related to cognition, 
as the process which gives rise to the operational congruence between an organism and its niche, that is the process that we distinguish in daily life either as either learned or instinctive knowing, is structural coupling [30].

Many attempts to develop Biological Closure, Structural Coupling and related concepts have been unsuccessful despite the use of advanced mathematical tools (see the use of Category Theory in Rosen [25, 31] and Nomura [32, or the use of Indicational Calculus by Spencer Brown [33], or Varela's formalization of autopoietic systems [34]. Mostly the aforementioned theories/models have ignored fundamental advances in modern mathematical physics. We have found that interesting conceptual advances can be achieved by considering such advances in parallel with notions of Biological Closure, Structural Coupling and the derived concept of Ecological Cognition.

\section{Biological Organization Principles}

Overview. Our theory, the Biological Organization Principles (BOP) framework, takes fundamental ideas of Autopoiesis and Cognition Theory -Biological Closure and Structural Couplingand blends them with basic and advanced concepts in Mechanics, Dynamical Systems, Probability and Statistics asserting them under a new light. Fundamentally, Biological Organization can be explained as a process continuously translating regularities of the medium into relational structure between the system's components. In technical terms, this fundamental translation process is performed by a single central core, based on advanced Stochastic Mechanics: the asymptotic evaluation of sample path integrals in a field of Markovian processes actively constrained by KAMtheorem. Several consequences are derived from this central core: first, it translates medium regularities into an organized structure endowed with cognition (system-niche duality). Second, it implements Biological Closure and Structural Coupling. Both concepts are broadly present in most theories about the biological organization and are fundamental in the ACT. Third, provides a framework-mechanism allowing system's progressive complexification. This basic operation builds incrementally more organizational structure adding more interdependencies inside the system and in connection with the system-medium interactions. Fourth, provides mathematical analysis using differential geometry to understand manifolds of processes and their topological interactions. Fifth establishes informational fluxes between medium regularities and ordered structure as it connects unpredictable -but regular- dynamics with dynamical self-ordered states. Sixth, its entails complex dynamical structures channeling motions from chaotic states to quasi-regular and integrable 
attractors.

Overall, Biological Organization Principles framework is a formal integration of biologically relevant concepts, in the setting of modern mathematical-physics, which also implies a change of paradigm in how we understand biological phenomena. We consider central theorems for living systems' organizational dynamics, that are also of widespread use for inanimate matter, shifting how we understand biological systems and its relation to their environment. More notably, the physical energy paradigm is extended to allow the transformation of chaotic regimes toward ordered relational states in systems of asymptotic integrability of Hamiltonian functionals, which defines living systems. Thus Biological Organization endows living systems with a mechanism that translates a self-constructive evolving non-linear system capable of situated anticipatory cognition. Under our viewpoint, fundamental aspects of biological systems can effectively be understood in light of these mathematical-physics ideas, which as a whole represents the Biophysics of Biological Organization. Hence, living organization dynamics and biology can be understood as a complex nonlinear dynamical system where its properties are the results of basic mathematical-physics concepts orchestrated by Biological Closure and Structural Coupling. In other words, understanding of the living organization as a complex nonlinear dynamical system, enable us to have a common language to mechanistically explain how living organization emerges from the same basic epistemological setting from where we understand mathematical-physical objects. BOP should not be interpreted as a mere physicalist view of living systems, as one of its cornerstones is the historical relationship between organism and medium. Thus BOP confronts the basic difference between complex inanimate physical objects and living systems.

In this section, we address BOP conceptual framework. First, we briefly overview the main developments leading to differential geometry and modern mathematical physics; sec. 2.1.1. We believe that the historical, as well as the conceptual paths, took on developing modern notions and the use of space-time and fields concepts are interesting to biology as they also depict a de facto example of robust theoretical construction. Nevertheless, if you are familiar with mathematicalphysics developments, you can pass directly to section 2.2.

We think that a good way to address biological organization is by defining components, systems, and dynamics as it is made in mathematical-physics; we introduce this approach in section 2.1.2. Once the components and the systems are clearly defined, the mechanical interactions and forces establishing the constraints on the time evolution dynamics of the system are then presented. 
We recall, the fundamental mechanics principle of least action in section 2.1 .3 and we finish the overview on mathematical-physics showing the emergence of the so-called deterministic chaos, that represents an extreme stage of dynamics, see section 2.1.4. We hope that these sections on differential geometry and mathematical-physics leads to a deeper interest in how these topics are central for a more comprehensive background towards a better understanding of the Biological Organization Principles framework.

In section 2.2 we begin the presentation of the mechanistic core of BOP that we termed Dynamical Closure Mechanism. First, we briefly overview how Biological Organization Mechaniç 3 opposes the trend to physical-mechanical chaos. Fundamental to this opposition is the phenomena known in biology as metaplasticity, which is a very peculiar mechanism guiding the dynamics of living systems under the constraints of Biological Closure and Structural Coupling. We will show that the biological metaplasticity in the Dynamical Closure Mechanism, allows for a closed diffusionpropagation perturbation in the organized interaction networks, section 2.3. Then we recast the use of the concepts of Biological Closure and Structural Coupling in the BOP framework. We overview DCM from microscopic and macroscopic viewpoints as both are relevant and broadly used in experimental and theoretical research in biology. Then, we start a step-by-step construction of DCM. We first define the Biological Organization space, coordinates systems and particles, section 2.3.2. The assembling of separate components and processes into interacting networks occurs as functional symmetries 'particles' reach steady states configurations, (sec 2.3.3). Next, we describe the geometry and dynamics of the manifolds formed by recursive iterations and embedding produced by DCM, 2.4, where we introduce the KAM-theoretical framework for Biological Organization. After a summarized overview of the Geometry and Dynamics formed by the recurrent operation of DCM, we describe the dynamics flow on DCM-Manifolds, section 2.4.1. One interesting aspect of BOP is its immediate use in discussing biologically relevant concepts related to niche definition, as well as aspects of biological evolution, biogenesis, and cognition. These aspects are discussed in section 2.4.2, where the interactions with the environment become recursive, and constraints the sample paths integrals defining the expected Biological Organization Hamiltonian. Therefore a particular historical lineage is established; entailing ontogeny, cognitive couplings and evolutionary dynamics. In section 2.4.3, we address the origin and evolution from the perspective of Biological Organi-

\footnotetext{
${ }^{3}$ we introduce this concept to differentiate the basic movements and forces occurring in the context of BOP
} 
zation Principles framework; we explain how the niche definition also entails, for longer scales of DCM operation, phylogenic-ecosystem couplings. We also discuss how DCM-operation positively moves towards increasing complexification from very basic proto-metabolism. We finish this section recasting autopoietic evolution by natural drift under the light of our Biological Organization Principles framework.

\subsection{Mathematical and Physical Preamble}

Mathematics and physics are natural stepping stones to approach a formal description of the living organization. Mathematics and physics are very technical disciplines, only partially present in the conceptual and methodological toolbox of biologists, therefore we offer a short overview on the main ideas and some history of the concepts needed to understand our proposal.

Whereas mathematics and physics initiated one of the most fruitful endeavors from where Nature (as conceptualized in XVIII century) emerges in the form of laws and simple equations while biology became estranged and set apart. One illuminating moment comes from Immanuel Kant, who made the statement in his Critique of Judgment: there will never be a Newton of the Blade of Grass because human science will never be able to explain how a living being can originate from inanimate matter. Mathematics, physics, and biology have evolved from 1790 when Kant criticizes the lack of formal tools in biology as well as the inherent teleology, found on living systems. Nevertheless, nowadays no clear path has emerged to overcome Kant's Newton of the Blade of Grass objection. Implicit in Kant's objection is the idea that the biological world is ruled by principles that are un-reachable from mere mathematical-physics laws. Thus to overcame this objection it is necessary to explicitly present the fundamental rules and laws that are deducible from mathematical-physics albeit they may include peculiarities as they are applied to living systems. Here we will present those necessary abstractions and rules need to understand living systems; they represent the core Biological Organization Principles framework.

\subsubsection{Differential Geometry and Topology}

Geometry is an integral component of mathematical-physics, for example, Cartesian systems of coordinates is a fundamental concept to Mechanics and Geometry (for interesting historical accounts see [35, 20, 36]). The concepts of the ordered set of positions coordinates; velocities and higher order derivatives become the basis of mathematical-physics. Fundamental figures were Riemann and Poincare who gave a modern theoretical outlook to the notions of space and manifolds in geometry 
[35]. Analytic Mechanics, developed by Lagrange, Hamilton, Jacobi, Liouville and Poisson became a geometrical mechanics, as global systems were described by Hamiltonian vector fields on the phase-space manifolds. Furthermore, the geometrization of Analytic Mechanics program lead by Poincare gave rise to modern symplectic geometry [35, 37, 38, 39, 40,

Our Biological Organization Principles approach should be thought as part of these geometric abstractions, as it includes theoretical and applied mathematics of Symplectic Geometry, Hamiltonian Systems, and Nonlinear Dynamics. We will show how our theoretical model gives new perspectives on the geometry of dynamical systems using the ideas of Biological Closure and Structural Coupling. A broad overview of Symplectic Geometry, Hamiltonian Systems, and Nonlinear Dynamics is not in the reach of this document, nevertheless, an introductory setting can be obtained from the following sources: [41, 19, 42, 43, 37, 44, 40, 45, 46]. We claim that modern theoretical thinking in biology requires to study Differential Geometry including fields as Manifolds, Lie Groups, Fibre Bundles, Topology and Gauge fields [37, 38]. Interestingly, a framework using differential geometry has been extended to biology to understand biomechanics and neuro-computing (see [47, 48, 49]).

Mechanical Systems. Newton's formulation of classical mechanics leads to the understanding of forces and movement; synthesized as the first, second and third Laws of Newton. In turn, JosephLouis Lagrange (1736-1813) gave the first example of a symplectic manifold in his studies of the motion of the planets under gravitational interactions, in the background of analytic mechanics (1808; [50, 51, 52]). Further developments by William Rowan Hamilton (1805-1865) replaced the uses forces by a geometrization entailed by the Hamilton Principle and the equations of motion of Hamiltonian mechanics.

Formal treatment of mechanical systems has advanced for more than three and half centuries building a robust framework (for the basics [53, 44]). Therefore, we think that Mechanics, (Classical, Statistical, Quantum, and Stochastic), plus the theory of Dynamical Systems are the necessary fields to understand Organization in Living Systems and we will use them to build our integrated Biological Organization Mechanics as a central process of Living Systems, in the form of the DCM model.

\subsubsection{Components, Systems and Dynamics}

The configuration of components, systems, and dynamics are central to any physical theory. Any glance to the many attempts to use physics approaches in biology shows how difficult this 
step could be. We believe that the constructive formulation of living system' phenomenology is possible following the same grounds used to handle physical systems under mathematical-physical approaches. Defining these entities, for biological systems, is rather important as living systems are characterized by a multitude of component classes with many interactions between them.

Elements. In classical mechanics, the elements are usually bodies, whereas in quantum mechanical descriptions they are usually the fundamental particles. These elements, are also defined by properties held by them: the mass of the body, the wave function of the particle, etc. Nevertheless, the properties of these elements are also related to the possible interactions between them [54]. For example, the electronic spin or the magnetic momentum, are relevant under the system properties in electromagnetic fields. In a practical sense, the elements are a vector of properties that allow them to interact in some theoretical manner. Organized systems, on the other hand, held order relations which can be totally different from the properties of the physical components as the important aspects must reflect the relations with other components. Thus, in our BOP framework, the elements hold two main properties: i) a relational order property defining the interactions with others components of the same kind; ii) functional features that allow the elements to perform a structure-function relationship for the organized system; we termed the fundamental objects as functional symmetries (we will defined more precisely in section 2.3 ). The definition of Biological Organization components dissociates organization from physical components, focusing instead on components that could be distributed across many types of molecules, organelles, cells, individuals, etc..

Systems. A set of elements interacting in some specific manner are usually defined as a system, thus a system is an interacting group of elements forming an integrated whole. Similarly, the elements of the system interact by specific features allowing the system as a whole to have emergent features. At the same time, some implicit or explicit methods, procedures of interactions and dependency of parts also construct the system. Every system has spatial and temporal boundaries, surrounded and subjected to influences by its environment.

In BOP the same logic for the system construction applies, but with the added dimension that the basic elements (functional symmetries) entail abstractions covering several levels of hierarchical organization. The interactions between functional symmetries allow the emergence of a wide spectrum of system features. The elements in the Biological Organization space (system), as we will 
see later, forms an organized system, that we will call a Hamiltonian Organization, distributed in a diverse heterogeneous physical structure of components implementing a closed mechanical organization, in the sense of Biological Closure, a quasi-integrable Hamiltonian system constructed by DCM. More interestingly, in the structure of the organized system, the history of recursive systemenvironment interactions will be incorporated in structural changes. These system-environment interaction dynamics is the basic mechanism of ecological cognition present in living systems. The structural modifications allow the systems to perform situated cognition using its own structural elements (self-reference) as a model of the external world.

Dynamics. Once the system and their elements are been defined the next step is to describe the system's evolution as the inescapable results of theoretical constraints and evolution laws. Nonlinear systems with complex and chaotic dynamics and self-organization are the natural interfaces to our theoretical point of view. Is at this respect that current Biology, with a strong emphasis in experimental science, arrives at basic ideas of dynamics and simple basic steady-state solutions, but barely uses more general contexts of long term stability, attractors and stability manifolds to interpret systems evolution. In the framework of BOP, dynamics has a fundamental role as the dynamical evolution of the system-environment interaction is incorporated in the organizational structure producing historically organized objects with cognitive capabilities. We will show that the central construction of DCM, allows the interactions of elements to follow the powerful KAMtheorem and its associated dynamics.

\subsubsection{Extremum Principles, Actions and Hamiltonians}

The extremum principles ${ }^{4}$ reflect successive stages of developments of Mechanics. The concepts entailed by them allow Mathematics to yield beautiful general formalism to the time evolution of physical systems. In the now classics works of Lagrange and Hamilton the fundamental notions of mechanics were abstracted to nurture the modern methods and principles of analytic mechanics [55, 42].

Fundamental is the contribution of Lagrange asserting the Least Stationary Action (LSA) principle. The LSA principle, also known as the least-action principle, comes to be an axiomatic

\footnotetext{
${ }^{4}$ Its history its also quite controversial (scandal); a detailed historical exposition of the extremum principles can be found in $[35,36]$.
} 
statement for physical systems on nature: namely that from many possibles ways to something to happens the observed one is the least. In other words, the observed instance is the one extremizing a physical quantity (in this case the action). Or as it was thought by Leibniz, physical laws of nature fulfills extremal principles for certain conservation quantities - [making our world] the best of all possible worlds [36]. Extremization appears first in calculus using the first and second order derivatives to find local maximum or minimum. Later these ideas were generalized, by Leibniz and Jacob Bernoulli, into Variational Calculus which finds, instead of simple values, functions that extremize certain mathematical expressions. Lagrange's fundamental contribution, later amended and clarified by Hamilton, was to interpret mechanics in terms of generalized coordinates instead of forces and positions. Under this approach system's invariants appear (like $\mathrm{E}=\mathrm{K}+\mathrm{V}$ ) opening the path to what we call today Symplectic Geometry $5^{5}$ a fundamental field that focuses on invariants produced by dynamical systems. BOP benefits from symplectic geometry by searching for invariants into the complex dynamics produced by living systems. Initials viewpoints in symplectic geometry are recognized to Carl Gustav Jacob Jacobi (1804-1851) and Hamilton's deep formulation of Lagrangian mechanics around $1835^{6}$.

The Euler-Lagrange equation, abstracts the dynamical behavior of free moving idealized particles in generalized coordinates, the LSA principle reads:

$$
\frac{\partial L}{\partial q}-\frac{\mathrm{d}}{\mathrm{d} t}\left(\frac{\partial L}{\partial \dot{q}}\right)=0
$$

Here $L$ denotes the Lagrangian functional which is extremized over the generalized coordinates $q$, and its derivatives, play important roles. From this, the Hamilton's principle develops this idea further by defining the following action functional,

$$
S[q]=\int_{t_{1}}^{t_{2}} L(q(t), \dot{q}(t), t) d t,
$$

For physical paths the variation $\delta S=0$ recovers the central Hamilton's equation of motion and defines the Hamiltonian functional, $H(q, p)$ relating generalized coordinates $q$ and momentum $p$ by:

$$
\dot{p}=\frac{\partial H(q, p)}{\partial q}, \dot{q}=-\frac{\partial H(q, p)}{\partial p}
$$

\footnotetext{
${ }^{5}$ the word Symplectic (meaning complex) was introduced by Hermann Weyl to differentiate from the geometry of complex numbers

${ }^{6}$ Hamilton expands and reformulates works of Galilei (1564-1642), Huygens (1629-1695), Euler (1707-1883), Lagrange, and Newton (1642-1727) on structure and behavior of orbits of planetary systems [50, 51, 36, 52].
} 
The two coupled differential equations describe the evolutionary dynamics of mechanical systems; corresponding to all the discrete point masses as well as rigid bodies within the tangible world!; BOP extends this framework to understand the organization of living systems.

In parallel with these developments in Analytical Mechanics modern geometry made major achievements for understanding dynamics. Sophus Lie along with Felix Klein revolutionized conceptions and approaches to geometry [56]. Lie's ideas of transformation groups and the associated algebras resonates in the Erlangen program by introducing the notion of symmetry properties as a core object in modern geometry 7 . Further developments of differential geometry by Elie Car$\tan$ leads to the current formulation of Hamiltonian mechanics in the frameworks of symplectic geometry.

Lastly, quantum physics pushed forward analytical mechanics at the beginning of the $\mathrm{XX}^{\text {th }}$ century by fundamental contributions of Erwin Schrodinger, Werner Heisenberg, Paul Dirac, and Richard Feynman. Schrodinger considerably expanded mechanics by considering probabilistic description of the state of a system. Since him, elementary particles do not have definite orbits but rather their position and velocity are described by density functions as in the famous equation relating changes in system states with the action of a Hamiltonian operator.

$$
\frac{h}{2 \pi} \frac{\partial}{\partial t}|\psi(\mathbf{r}, t)\rangle=\hat{H}|\psi(\mathbf{r}, t)\rangle
$$

This equation states that, for quantum systems the system state is represented at time $t$ by a function $(\psi(\mathbf{r}, t))$ whose time-derivative can be computed as the action of a Hamiltonian $(\hat{H})$ over the system state. Although this formulation incorporated several less well known operations as the Poisson brackets, the fundamental ideas of probability density functions along with gradients, curls, trajectories and other mathematical operations (Poisson, Lie bracket and so on) it extends Mechanics to stochastic systems (see for example [57]).

Feynman further generalized the LSA principle by explicitly giving a procedure to compute $\psi(x, t)$ by considering all possible trajectories linking two states as in:

\footnotetext{
${ }^{7}$ This approach appeared similarly strange and obscure to contemporaries. Symmetry properties are the main focus of modern geometry, the points, lines, etc. are no longer the main objects. These symmetries are described by properties of groups, a previously totally algebraic concept developing the theory of so-called Lie-groups and Lie-algebras.
} 


$$
\begin{gathered}
\psi(x, t)=\frac{1}{Z} \int_{\mathbf{x}(0)=x} e^{i S(\mathbf{x}, \dot{\mathbf{x}})} \psi_{0}(\mathbf{x}(t)) \mathcal{D} \mathbf{x} \\
S(\mathbf{x}, \dot{\mathbf{x}})=\int L(\mathbf{x}(t), \dot{\mathbf{x}}(t)) d t
\end{gathered}
$$

With these equations, Feynman, besides developing Quantum Electrodynamics (QED), introduces novel variational methods relying on Lagrange densities the field theoretical counterpart of the Lagrangian for discrete systems to reveal equations of motions as averages of microscopic quantum trajectories. Additional fundamental developments come in hyperbolic-parabolic-ellipti 8 PDEs and probability theory by the mathematical-physics school in Russia. In effect, beginning with Lyapunov and Markov'9 and then with, Kolmogorov 10 Arnold, Chirikov and Nekhoroshev key results and methods, including the famous KAM theorem, was developed to understand nonlinear dynamics and probability theory [45, 58, 43, [59, 47, 41].

In summary, the Principle of Least Stationary Action with Variational and Stochastic Calculus are important cornerstones in the development of Mechanics and they will play a fundamental role in our Biological Organization Principles framework. In particular, at the microscopical level, stochastic path integrals will be shown to be essential, as a representation of the formation of Biologically expected closed Hamiltonian in non-homogeneous media. The perturbation of the nonhomogeneous media via recurrent interactions by the system allows to include in the time evolution dynamics the history of interactions as the operation of KAM-theorem.

\subsubsection{Deterministic Chaos and Chaotic Behaviour}

The mechanical behavior of complex systems can be highly non-linear and, as discovered by Poincare, the time evolution can be very sensitive to initial conditions. System dynamics are determined by the equations of motion (Hamilton's equations) or movement laws (Newton's laws), however, if the interactions are not linear, two very near initial conditions may lead to huge departures in their deterministic trajectories. In such conditions, trajectories do not have the certainty of determinism although deterministic equations control system dynamics: this phenomenon is termed

\footnotetext{
${ }^{8}$ responsible for the main features in evolutionary dynamics: propagation, diffusion, and oscillation

${ }^{9}$ Students of Pafnuty Chebyshev; father of the pre-revolutionary Russian mathematical school, also Liouville and Hermite's friend.

${ }^{10}$ intellectual grand-grandson of Liouville and Weierstraß
} 
deterministic chaos. Chaos introduces limitations in our ability to accurately predict the evolution of a dynamical system at different scales [58]. Nevertheless, these systems exhibiting chaos can still be studied with the tools of modern Dynamical Systems Theory: phase spaces, vector fields, manifolds, and Hamiltonian (Lagrangian) dynamics. Additionally, we can also have numerical and theoretical methods to study chaotic attractors [60, 58]. Interestingly, Integrable Hamiltonian systems can be perturbed without leading to chaotic, stochastic attractors. These conditions of slightly perturbed systems are the theoretical conditions established by the Kolmogorov, Arnold, and Moser (KAM)-theorem. Notably, as we will see shortly, KAM-theorem opens the path to include the history of recurrent interactions as constraints defining the stability and evolution of living systems. An important parameter of KAM-theorem is the perturbation parameter $\epsilon$ which sets the system's Hamiltonian fluxes as deterministic or chaotic regimes. However, just before the critical perturbation value $\left(\epsilon_{c}\right)$, chaotic and KAM-attractor coexist in a KAM-torus. This constrained, or soft-chaos, shows diffusion fluxes that continuously move the interface of self-organized patterns and chaotic attractors at several scales. Mathematical-physics of this phenomena is very well studied in physics; from elementary particles to cosmology; laser physics, quantum computing, meteorology, fluid dynamics, etc., see for example its application to chemical reactions [60], and we will extend it to the biological organization. In the following sections we will introduce, the core mechanism actively forcing a biologically organized dynamics by discussing how nearly closed, quasiintegrable Hamiltonians are formed from non-homogeneous stochastic perturbations. Starting from pure physicochemical grounds [60], organization emerges from chemical reaction networks which, by Structural Coupling, dynamically reproduce and stabilize their structural physical-counterparts constructing a quasi-integrable Hamiltonian, reflecting Biological Closure and representing Biological Organization.

\subsection{Organizing Chaos via Dynamical Closure and Structural Coupling}

One fundamental property of living systems is their ability to oppose the physical trend to disorder. The second law of thermodynamics has been a huge unsolved obstacle to the explanation of living systems. Schrodinger analysis on What is Life [61] introduces negentropy as a key concept to represents the translation of order from physics into living systems (organization, structure, and function); how this actually occurs however still is a mystery. An answer to this old question is obtained from BOP, specifically by DCM (section 2.3), by considering that organized systems are 
embedded in a medium and the interactions system-environments are: i) actively constrained, ii) incorporated in the organization dynamics and iii) follows KAM-theoretical dynamics. Chaotic behavior emerges as a consequence of the system's non-linearity and environmental perturbations; whereas Biological Organization Hamiltonian, a direct consequence of Structural Coupling and Biological Closure, constraints and departs from chaotic behavior. The fundamental mechanism building the Hamiltonian responsible for Biological Organization is DCM which utilizes a mechanism akin to equation (5) and (6) to build a Biological Hamiltonian. This construction averages systems states (expectation) across sets of (stochastic) trajectories modulated by recurrent interactions (perturbations) with its medium. On this context, Biological Closure allows to form mathematically expected (quasi-) closed stochastic paths samples from interacting networks. On the other hand, Structural Coupling defines the perturbations to be incorporated into the Biological Hamiltonian as a historical dynamics. Then three important features of organisms are predicted by Biological Organization Principles; niche definition, cognitive computation and conservative evolution of the organization.

\subsubsection{Biological Organization}

Biological Organization is a concept that needs to be clearly defined to properly understand our proposal. The duality between form and function its a central trait in many approaches; as well as the notion of teleology as a final cause of form and function. Usually in Biology the concepts of form and function are thought to represent two complementary facets of relevant biological entities associated to organization; for example, DNA (as form) and information (as function).

However, the Biological Organization can be thought of as a specific domain of relational phenomena. In Biological Organization Principles framework, Biological Organization, emerges as the operation of DCM (next section), entailing mechanical and dynamical components, to unfold the mutual constraints on structure and function, leading to exhibit a given BIOLOGICAL FORM. Additionally, we consider that Biological Organizationand structure of living systems are historical processes reflecting the interactions between system and environment.

How DCM operates is the core of the next section; there we consider the organization as a formal concept acting in an idealized formal mathematical ORGANIZATIONAL SPACE. 


\subsection{The Dynamical Closure Mechanism, DCM}

The core of our treatment consists of the Dynamical Closure Mechanism, (DCM); which describes, assembles and operates the central basic biological mechanism defining Biological Organization. In other words, the DCM - combines Biological Closure and Structural Coupling entailing the basic biological organization mechanism operating in living systems. As mention before any theory facing the problems of Biological Organization must deal with primitive objects like Elements, Systems, Dynamics. In our case, the basic elements are defined using the notion of symmetry in the formal sense of a structure-preserving transformation. Thus the elements, in BOP, are processes that preserve fundamental functions, thus they are termed functional symmetries. It is important to underline that symmetry, in the formal sense, are usually transformations that preserve invariants (e.g. the symmetries of the hydrogen atom are rotations around the center of the atom, which form the Lie group $S O(3, R)$.) BOP uses this vision of symmetry in a dynamical context. For the case of biological organization, the symmetry is revealed as a transformation preserving the organizational structure which is made of biological functions. Therefore functional symmetries, are objects abstracting relational properties in the organizational realm. Functional symmetries are different from the physical realm of material objects as atoms or molecules. The relation of functional symmetries to the material physical objects is specified by the structure-function relationships and it is usually studied in experimental biology. The functions, as for example: substrate-product, excitationinhibition, active-inactive states, etc., are the operation-transformations defining symmetries of the objects (see section 2.3.2). Then, the components and interaction networks constituting living systems are functional symmetries driven by DCM and they are the dynamical backbones preserved in the Biological Organization.

Biological Organization Mechanics. As we mention, BOP framework takes the notion of Biological Closure and Structural Coupling as central ideas to define mechanistically the operation, process, and elements which define Biological Organization. Briefly, we address them in the context of our framework.

Biological Closure. As we have seen in section 1.1, Biological Closure is a multifaceted concept. In our mechanistic account of biological organization, biological closure is the existence of closed path trajectories which are essential to the formation of integrable and quasi-integrable Hamiltonian dynamics. Biological closure, also become a prominent feature on living systems as it enables systems 
to become robust by compensating recurrent streams of perturbations. In the basic definition of the organizational elements (functional symmetries), biological closure also serves to form conjugated pairs, the momentum to the position-activity variable, as it systemically co-variates with changes in functional symmetries. Biological closure can modify components and processes without changing the system's organization. Thus the recurrent structure of each perturbation stream can be incorporated, via homeostatic plastic changes, into system's organization.

Structural Coupling. As was discussed, the structural coupling is a key notion in how systems are coupled to their environment. In Maturana's own words we read,

I have called the dynamics of congruent structural changes that take place spontaneously between systems in recurrent (in fact recursive) interactions, as well as the coherent structural dynamics that result, Structural Coupling [30].

More generally, Structural Coupling refers to the congruent changes and emerging dynamics that occur by the recurrent interaction between system and environment. Nevertheless, in the intellectual corpus of Autopoietic theory, there is no explicit mention of how this spontaneous coupling to the medium occurs, albeit many features and consequences of Structural Coupling have been derived [30]. We propose DCM as the mechanism underpinning Structural Coupling. As DCM preserves functional symmetries while also incorporating, into the system's structure, plastic changes correlated with the history of recurrent interactions between the organism and its medium, it becomes a framework to understand Structural Coupling in terms of a formal definition.

Structural Coupling appears as operating the extremizing features in the functional construction of the organizational dynamics. Likewise, it is also embedded in the asymptotic evaluation and minimization of the perturbations which are evaluated via KAM-theorem. Therefore DCM, which is the mechanism of Structural Coupling is also central in producing Biological Closure by implementing the trajectories of organizational invariance. DCM, then become a particular type of mechanics (Biological Organization Mechanics), encompassing complementary Deterministic and Stochastic viewpoints; next, we present them briefly. Furthermore we will recover Biological Organization in the form of the Biological Organization Hamiltonian, $\mathcal{H}^{\mathcal{B}}$, which has deterministic $\left(\mathcal{H}_{d t}^{\mathcal{B}}\right)$ as well stochastic $\left(\mathcal{H}_{s t}^{\mathcal{B}}\right)$ formulations. 


\subsubsection{Microscopic and Macroscopic DCM}

DCM \& Microscopic Stochastic Mechanics. Microscopically DCM is constructed by interacting networks of Markovian processes, each of one representing the elementary dynamics of a single functional symmetry, in the setting of Stochastic Mechanics. Recently, it has been shown how results from Classical Mechanics and Dynamical Systems map into stochastic concepts [62, 57], a mapping particularly relevant for DCM.

Basically in DCM, interaction networks of stochastic processes - i.e. coupled functional symmetries in the form of Markovian chains - close their sample paths resulting in recurrent diffusive dynamics (governed by nonlinear forward and backward Kolmogorov equations) coupled to re-entrant perturbations from the environment. Thus, the stochastic trajectory pathways (Markovian chain samples) and associated probabilities densities, of the functional symmetries, reflect the changes in structure and function caused by the recurrences of interactions with the medium. Therefore DCM entails a underlying Bayesian Inference mechanism acting on the recurrences of interaction system-medium which become incorporated in the organization by a continuous diffusion process.

Microscopically, the stochastic fluctuations in the interaction networks of coupled functional symmetries propagate the organizational dynamics which is defined as the statistical expectation of integrable Hamiltonians, $\mathcal{H}_{s t}^{\mathcal{B}}$. Therefore, under DCM stochastic fluctuations form closed samples paths assembled by recurrent interactions between the engaged functional symmetries and the medium and, by this mechanism, implement Structural Coupling.

It must be noted that stochastic DCM operates also as an embedding mechanism acting across hierarchical levels of Biological Organization. Microscopically, stochastic recursive DCM operation assembles the full metabolic network reaching self-construction of components, resulting in autopoiesis. Whereas that at mesoscopic levels DCM defines the multicellular and systemic organism organizational interactions, while macroscopic levels account for niche interaction as well as ecosystems organizations. Interestingly, DCM embeddings across hierarchical Biological Organization levels can be approached by port-Hamiltonian frameworks [63]. In this case, the underlying connected networks (the Dirac structure of port-Hamiltonians) are continuously adjusted by the perturbations such as an increasingly smoothing of the connected port-Hamiltonian embeddings is achieved. As a result, DCM evokes naturally a port-Hamiltonian framework. 
DCM \& Macroscopic Deterministic Mechanics. The principle of least stationary action is at the core of Classical and Quantum mechanics as it defines the Hamiltonian describing the evolution of systems in terms of their constants of motion. Then, Hamiltonian integrable functionals completely determine the laws of motion associated with deterministic systems. Accordingly, we will use this mechanical description as a central core for DCM acting in its deterministic setting by the deterministic Biological Hamiltonian, $\mathcal{H}_{d t}^{\mathcal{B}}$. A Hamilton-Lagrange formulation of the Biological Organization Principles framework, based on the ideas of Biological Closure and Structural Coupling, implements an Autopoietic Mechanics for living organization. Therefore, the living organization resulting from DCM displays an important class of invariants: closed and quasi-periodic trajectories in phase space. Thus DCM induced dynamics should be analyzed in terms of invariants in Symplectic manifolds opening the approach to use Symplectic Methods and Lie Theory. A cornerstone of the BOP and in the DCM is the fundamental theoretical result by Kolmogorov (1954), Arnold (1963) and Moser (1962); known as the KAM-theorem; which establishes when a dynamical system is endowed with a Complex Attractor Manifold. The mechanistic core implementing DCM, in the deterministic or stochastic settings, forms quasi-integrable Hamiltonians $\left(\mathcal{H}^{\mathcal{B}}\right)$ reflecting Biological Closure and implementing KAM-theoretical dynamics in the organizational manifolds by Structural Coupling. Thus stochastic and deterministic DCM frameworks, implement Biological Closure and Structural Coupling, endowing living systems with organizational conservation, cognition, and evolution.

\subsubsection{Biological Organization Space}

The Elements, the Functional Symmetries: relational points in generalized coordinate system. First, the elements in biological organizations maintain order relations between themselves which are given by functional features assembled by the operation of DCM. Therefore, the relevant elements in Biological Organization are endowed with structure-function relationships as is usually thought in biology (in the physiological, ecological and evolutionary senses). The different configurations of the structure-function relationships, i.e. functional symmetries, play a central role in DCM and are represented by transformation schemes under microscopic equilibrium:

$$
(1-x) \underset{\beta}{\stackrel{\alpha}{\rightleftarrows}} x
$$

In this binary scheme, $x$ represents a measure of the functional symmetry state while " $1-x$ " is 
$x$ 's complement; and the time evolution of $x$ is given by the rate equation:

$$
\dot{x}=\alpha(1-x)-\beta x
$$

Biological Organization is thus deployed as an ensemble of mechanical and dynamical interactions among functional symmetries under DCM. For example macro-molecules in metabolic networks; species in the ecological networks; neurons, muscles, glands, in excitability networks and antigenic production and lymphocyte populations for immune networks. It should be noted that the actual physical structure of the functional symmetries reflects the constraints of DCM leading to their specific biological form. Then, elements at the organizational level are an abstraction of structure-function endowed with magnitude-position $(x=q)$, rate-momentum (derivatives, $\ddot{x}=p$ ) and some systemic $(\alpha, \beta$; mass-action) variables, all of which are system dependent, forming a system under Biological Closure. Therefore the Biological Organization space, implemented by the intrinsic recursivity of DCM, is associated with vector and phase spaces of high dimensionality (one dimension for each functional symmetry). This abstract organization space is a Hilbert space defined by orthogonal basis, given by generalized coordinates for position amplitude $(x)$; whereas the phase space combines the rate/velocity of movement $(\dot{x})$ and the systemic canonically conjugate momenta $(\ddot{x})$

\subsubsection{Stochastic Mechanics and Probability of Functional Symmetries}

Functional symmetries can be analyzed using stochastic or deterministic viewpoints each of them conveying distinct aspects of Biological Organization. Following standard techniques in probability theory, a short version of our DCM for the stochastic setting is as follows.

Each functional symmetry associates with a random variable $(Z)$, representing position/amplitude in the form of a probability density function $f(z)$, whereas its rate/movement associates to the transition probabilities between states (i.e. $P\left(z_{n} \mid z_{n-1)}\right)$ in a Markovian chain. For the case in equation (7), a binary states of symmetries ${ }^{12}, Z \in\{0,1\}, P(1)=1-P(0)$, and $P(1 \mid 0)=P(x \mid(1-x))$.

\footnotetext{
${ }^{11}$ It should be noted that although A Hilbert space is adequated for the most simple configuration of functional states, higher order interactions can lead to a Fock space as its is the case for some non-classical descriptions involved in cognition and decision making, were the neurological basis follows non-kolmogorovian statistics 64

${ }^{12}$ Dynamical organization based in functional symmetries must be understood using discrete and continuous theories on random variables. A random variable $(X)$, is described by its distribution density function $f(x)$, the probability
} 
Thus the biological organization space containing a lattice/network of coupled variables $\mathbf{X}=$ $\left(X_{1}, . ., X_{k}\right)$ with $X_{k} \in\left\{X_{k} \Rightarrow f^{k}\left(Z_{i}\right) ; k, i \in \mathbb{Z}_{+}\right\}, k$-number of objects and $i$-symmetries, which represent the underlying functional symmetries. The temporal evolution of each density function $f^{k}(z)$ is governed by multidimensional Einstein-Smoluchowski-Kolmogorov-Chapman (ESKC) relations, which for the case in (7) associates its evolution law as it's in equation (8). More generally in DCM, the ESKC relations frame the evolution of $\mathbf{X}$ in time, $\mathbf{X}(t)$; the probabilistic coupling (conditional probability, $P\left(X_{k-1)} \mid X_{k}\right)$ to establish directed graphs shaping the most probable network structure between interacting functional symmetries. The ESKC relations are essential as they imply the $\mathcal{H}^{\mathcal{B}}$ as expectations of samples paths of Markovian chains of $\mathbf{X}$ : a fundamental concept to Biological Organization Principles framework. We hypothesize that due to Biological Closure and Structural Coupling the expected Hamiltonian, recovered by the Integral Paths method in ESKC, $\mathcal{H}^{\mathcal{B}}$ become conservative quasi-integrable.

The evolution, trajectory of $\mathbf{X}(t)$, between $t_{0}=0$ and time $t=\tau$ is given by the changes in the transition probability $p\left(\tau, x_{k} \mid t_{0}, x_{k}(0)\right)$ known as the Forward Kolmogorov-Chapman equation (FKCE), describing the process as a jump-propagation wave in a high probability flux:

$$
\partial_{\tau} p\left(\tau, x_{k} \mid t_{0}, x_{k}(0)\right)=-\partial_{x_{k}}\left[\alpha_{\tau}\left(x_{k}\right) p\right]+\frac{1}{2} \partial_{x_{k}}^{2}\left[\beta_{\tau}\left(x_{k}\right) p\right]
$$

This partial differential equation, also known as the Fokker-Planck equation, computes the future values of $p\left(t, x_{k}\right)$, parameterized with drift $\left(\alpha_{\tau}\left(x_{k}\right)\right)$ and diffusion components $\left(\beta_{\tau}\left(x_{k}\right)\right)$ for the underling Markovian chains in which probability flows across the networks of interacting functional symmetries from time $t_{0}$ to $t=t_{0}+\tau$.

A complete description of DCM dynamics requires to compute, starting from time $t_{0}$, the past probabilities $p\left(t=t_{0}-\tau, X_{k}\right)$ also in term of drift and diffusion components: this is the so-called Backward Kolmogorov-Chapman Equation (BKCE), (also known as the Feynman-Kaç equation).

$$
\partial_{-\tau} p\left(t, x_{k} \mid \tau, x_{k}(0)\right)=\alpha_{\tau}\left(x_{k}(0)\right) \partial_{x_{k}(0)} p+\frac{1}{2} \beta_{\tau}\left(x_{k}(0)\right) \partial_{x_{k}(0)}^{2} p
$$

that $X$ lies in the interval $[a, b]$ is computed by $\left.P(a \leq X \leq) b=\int_{a}^{b} f(x) d x\right)$. Its statistical moments by $\mu_{X} / E(X)$ (expectation) and $\sigma_{X}$ (standard deviation). If $X$ is well represented by a countable number of values $x_{i}, f\left(x_{i}\right)$ has the properties, $P\left(X=x_{i}\right)=f\left(x_{i}\right), f\left(x_{i}\right) \geq 0, \sum_{i} f\left(x_{i}\right) d x=1$; with $\mu_{X}=E(X)=\sum_{i} x_{i} f\left(x_{i}\right), \sigma_{X}=\sqrt{E\left(X^{2}\right)-\mu_{X}^{2}}$ For $f(x)$ piecewise continuous functions: $P(a \leq X \leq b)=\int_{a}^{b} f(x) d x, f(x) \geq 0, \int_{-\infty}^{\infty} f(x) d x=1$; with $\mu_{X}=E(X)=$ $\int_{-\infty}^{\infty} x f(x) d x, \sigma_{X}=\sqrt{E\left(X^{2}\right)-\mu_{X}^{2}}$ 
Thus FKCE and BKCE equations associate a perturbation route of micro-states transitions as a wave in the probability fluctuation of each functional symmetries.

Thus now we can introduce a fundamental idea of DCM, the use of sample path integrals in the formation of Biological Organization. The path integral method, also used in classical and quantum mechanics, serves to compute the Hamiltonian functional as the expectation from the probability densities associated to trajectories in the space of random variables $(X)$ connecting states $E_{0}$ (at $\left.t_{0}\right)$ and $E_{t}($ at $t)$ 65].

$$
\left\langle H_{s t}\right\rangle_{x(t)}=\mathcal{F}_{\left(t_{0}\right.}^{t]} e^{-S^{\dagger}}\langle\langle P\rangle\rangle_{x(t)}
$$

with,

$$
\begin{aligned}
\langle\langle P\rangle\rangle_{x(t)} & =\sum_{n=0}^{\infty} \frac{x^{n} e^{-x}}{n !} P(n) \\
S^{\dagger} & =\int_{t_{0}}^{t} d \tau\left[i q \partial_{\tau} x-Q_{\tau}^{\dagger}(x, i q+1)\right]
\end{aligned}
$$

The path integral representation of the evolution of the system is considered cornerstones and commonly used in the formulation of all mechanics (Classical, Quantum, Stochastic; [57, 66, 67]).

The derivation of equation (11) and some other insights can be found in [47, 68, 65, 66, 67], nevertheless a rough approximation can be grasped as follows. For each trajectory $E_{0} \rightarrow E_{t}$ a Markovian chain, associating coupled functional symmetries, represents an Action functional $\left(S^{\dagger}\right)$. Following from the LSA principle, $S^{\dagger}$ entails an extremizing functional of the underling conditional probabilities in the Markovian chain. Thus the trajectory taken by the system is the grand average of the ergodic excursion of probabilistic trajectories weighed by $S^{\dagger}$. The expectation of the probabilistic-action trajectory is the following stochastic Hamiltonian of the system, without external perturbations, represented by:

$$
\left\langle H_{s t}\right\rangle_{x(t)}=\mathcal{F} e^{-S^{\dagger}}\langle\langle P\rangle\rangle_{x(t)}
$$

Then, taking further the elements present in the construction of this physical Hamiltonian emerging by stochastic interactions (a normal situation in many branches of Physics), we can develop the features associated with living systems. In living systems, in contrast to non-biological systems, the history of perturbations/interactions between the system and its medium becomes incorporated in the Biological Hamiltonian functional $\mathcal{H}_{s t}^{\mathcal{B}}$. Thus in DCM, the fundamental mechanism of Structural 
Coupling, the external perturbations are incorporated in the formation of $\mathcal{H}_{s t}^{\mathcal{B}}$ and therefore shaping the organization. As we mention before Forward and Backward KCEs constructs, by plastic diffusion minimization over the set of functional symmetries associated to system-medium interactions, the expected quasi-integrable/integrable $\mathcal{H}_{s t}^{\mathcal{B}}$. This is a plasticity mechanism continuously operating in the stream of recurrent system-medium interactions to form trajectories of mechanical actions. Moreover, in BOP framework $\mathcal{H}^{\mathcal{B}}$ performs Biological Closure as an accelerated instance of Poincare's recurrence theorem aided by the meta-plasticity implicated by system-medium interactions.

Biological closure constrains the evolution of the system such that $\left\|E_{0}-E_{t}\right\| \leq \epsilon$ with $\lim _{t \rightarrow \infty} \epsilon=0$; as a consequence the biological Hamiltonian, $\mathcal{H}^{\mathcal{B}}$, approaches a symplectic form. Then, equation (11) used in our Biological Organization Principles framework defines $\mathcal{H}^{\mathcal{B}}$, which becomes,

$$
\left.\mathcal{H}_{s t}^{\mathcal{B}}=\lim _{\epsilon, t \rightarrow 0, \infty}^{\left\langle H_{s t}^{\dagger \circledast}\right\rangle}\right\rangle=\oint_{E_{0}}^{E_{t}} e^{-S^{\dagger \circledast}}\left\langle\left\langle P^{\dagger \circledast}\right\rangle\right\rangle_{x(t)}
$$

The symbol represents the asymptotic stochastic integral when Biological Closure and Structural Coupling are operating in the formation of the Hamiltonian functional, $\mathcal{H}^{\mathcal{B}}$. $\epsilon(t)$ represents the probability density of the perturbations induced by the external medium. Then the integrand part is further expanded as:

$$
\left\langle\left\langle P^{\dagger \circledast}\right\rangle_{x(t)}=\left\langle\left\langle P^{\dagger}\right\rangle\right\rangle_{x(t)} \circledast \epsilon(t) ; \text { and } S^{\dagger \circledast}=S^{\dagger} \circledast \epsilon(t)\right.
$$

where $\circledast$ is the convolution operator. Importantly, the perturbation $\epsilon(t)$ reflects the impacts on the functional symmetries, by translating the recurrence of the physical world into the structure of Biological Organization. Therefore, the perturbation acting in the Biological Organization is the convolution of $\epsilon(t)$ with $S^{\dagger}$ and with the probability expectation $\left\langle\left\langle P^{\dagger}\right\rangle\right\rangle$ as in equation (11). Thus $\mathcal{H}^{\mathcal{B}}$, in the stochastic stage, can be understood as composed of a limit computation in the stochastic path integrals and a convolution with the physical world represented by the physical Hamiltonian $\mathrm{H}(q, p)$ as is stated in equation 13.

$$
\mathcal{H}_{s t}^{\mathcal{B}}=\lim _{t \rightarrow \infty}\left\langle H_{s t}^{\dagger}(\epsilon(t))\right\rangle+\epsilon(t) \circledast \mathbf{H}(\mathbf{q}, \mathrm{p})
$$

Therefore, equations (12) and (13) represent DCM formal central core, they introduce a new perspective in quantitative biology as they permit to use KAM theory (theorem) to understand the dynamics of biological systems by focusing on how the organism-medium interactions define 
biological organization. As equation (13) shows, physical interactions, the component $\epsilon(t) \circledast \mathrm{H}(\mathrm{q}, \mathrm{p})$, becomes incorporated into biological organization (i.e. into the Hamiltonian $\mathcal{H}_{s t}^{\mathcal{B}}$ ). In this way recurrent system-environment actions become incorporated into systems structure and leads to cognition, as anticipated actions in a mechanical flow.

\subsection{Geometry and BOP Dynamics}

From the previous stochastic analysis an integrable Biological Hamiltonian, obtained as a limit, $\mathcal{H}^{\mathcal{B}}$, results from DCM recursive operations. $\mathcal{H}^{\mathcal{B}}$ is also the result of an expectation along sample stochastic paths and is a smooth differentiable Hamiltonian. We now address how this basic result determines higher order organizational dynamics in living systems. The deterministic setting of DCM establishes the classical mechanics approach in the Biological Organization Principles framework. Although differential geometry and other mathematical theories can be used to extend BOP, as for example Lie theoretical developments for its analytic formalism, we will focus on some general aspects of differential geometry and related tools.

Mechanical systems evolve governed by evolution laws which are abstracted in the form of Hamiltonian functionals. Physical systems, quantum or classical, use Hamilton-Lagrange descriptions, along with Path Integrals, to describe their time-evolution by extremizing the trajectories defining the interactions of the system. For BOP, the stochastic interactions between biological systems and their medium yield a resulting Hamiltonian, $\mathcal{H}^{\mathcal{B}}$, that parallels the classical description of the mechanical system (like mathematical-physics Hamiltonians, $\mathrm{H}(\mathrm{q}, \mathrm{p})$ ). In this regards, the more complete description of mechanical systems is achieved by Dynamical Systems supported by Differential Geometry. Likewise, Lie transformation theory ties together an abstract representation of geometrical fluxes and analytical expressions into an abstracted totality. Differential Geometry and Lie-theory represent parallel constructions fundamental to the development of Biological Organization Principles framework. The geometry of dynamical systems establishes the flows and fields of mechanical actions as relationships between the configuration manifolds and the tangent and cotangent bundles. In these manifolds, the interactions of the underlying functional symmetries set their steady states trajectories and recursive embedding as result of DCM.

A fundamental notion for the geometrical and topological frameworks behind BOP is that living systems' dynamics falls into stables N-dimensional Tori (N-Tori) manifolds of interacting functional symmetries networks. The N-Tori, assembles in port-Hamiltonian structures the smooth 
concurrent operation of DCM; enabling the physical structures supporting the higher abstraction of non-homogeneous energy fluxes (which is the standard situation in biological systems) [63]. This continuous operation of DCM lead to form an organized structure constrained be the systemmedium interactions, which as a historical process forms $\mathcal{H}_{\left\langle\epsilon\left(t_{0}\right)\right\rangle}^{\mathcal{B}}$, a self-constructed lineage by the expected system-medium interactions represented by $\epsilon\left(t_{0}\right)$, the history of interactions starting at $t_{0}$. In biology, the energy fluxes form hybrid subsystems ranging from electrochemical potentials (in physicochemical reactions) to biomechanical forces in muscles that smoothly interact to perform the physical actions entailed by $\mathcal{H}^{\mathcal{B}}$. The inherent recursivity of DCM generates a re-enaction of trajectories of interactions with the medium that become entailed in $\mathcal{H}^{\mathcal{B}}$. Therefore, an organizational lineage of historical interactions is also subsumed in the formation of $\mathcal{H}^{\mathcal{B}}$. These lineages evolve (from the physical molecular level) to construct higher-order structures incorporating new recurrent perturbations from the medium. The different biochemical and anatomical morphologies of an organism are continuously shaped, and hence they also transform its overall organization. As DCM continuously operates, multiple instances begin to work in parallel, for example, different biochemical pathways; different neuronal networks; different cellular clones of the immune system, etc. This parallel instances of DCM approach the operation of a deterministic Hamiltonian as all these stochastic instances collapse their sample paths into an integrable Hamiltonian $\left(\mathcal{H}_{d t}^{\mathcal{B}}\right)$.

Thus, similarly to the stochastic DCM (13), the deterministic integrable Hamiltonian constructed by DCM is formally represented by,

$$
\mathcal{H}_{d t}^{\mathcal{B}}=\mathcal{H}_{\left\langle\epsilon\left(t_{0}\right)\right\rangle}^{\mathcal{B}}+\epsilon(t) H(I, \theta)
$$

The deterministic DCM, incorporates the recursive transformation of an initial Hamiltonian $\left(\mathcal{H}_{\epsilon\left(t_{0}\right)}^{\mathcal{B}}\right)$ which evolves perturbed by the action from the medium of a physical Hamiltonian (mechanical systems in action-angle coordinates $H(I, \theta)$; one of its canonical transformations of $H(q, p))$. Such formal system has been studied by what it is actually known by the KAM theory. In effect, first with Kolmogorov's result in 1954, for a perturbation series of Diophantine expression; and after that, Arnold (1964) and Moser (1962) extending Kolmogorov results to more geometrical settings. Originally, KAM theory shows that nonlinear dynamics of conservative integrable systems, when subjected to small perturbations, remains quasi-integrable. Thus, a non-integrable Hamiltonian $\left(H=H_{0}+\epsilon V\right)$, results from a nonlinear integrable Hamiltonian $\left(H_{0}\right)$, coupled to a generic perturbation $(V)$ by a small coupling dependency $(\epsilon)$. The dynamics of an integrable, non-degenerate 
Hamiltonian, $H_{0}$ lies in the N-Tori. Whereas, for its perturbed systems most of the trajectories become bounded by the KAM-surfaces (KAM-tori), which are defined by $n$ true constants of motion and $N-n$ quasi constants of motion. As the size of the perturbation $\epsilon$ increases KAM-surface are modified until eventually being destroyed, due to the increase in quasi-constant of motions.

\subsubsection{Dynamics and Flows in the DCM-Manifolds}

Hamiltonian functionals relate directly to the equations of motion for mechanical systems; nevertheless, we will turn to their geometrical interpretation and relations to KAM theory. Biological organization requires the geometrical interpretation of the Hamiltonian rather than the classical energy paradigm 13 widely used in physical systems. In a geometrical sense, Symplectic Geometry provides an illuminating understanding of Hamiltonians as they are interpreted as generalized high dimension invariants, not only as of the total energy of a system. In the case of Biological Hamiltonians, as they exist in high dimensional spaces, is the Symplectic Geometry's interpretation the more useful than the usual energetic interpretation. Additionally, the fundamental notion of geometric invariants is even more important as it is associated with the dynamical transformation of the Biological Organization during system-medium interactions. Under this perspective, the operation of DCM is associated with the formation of manifolds in symplectic geometry; DCM-manifolds. These DCM-manifolds when perturbed become continuously transformed into KAM-manifolds.

To visualize the dynamics, we will mostly use a low-dimensional DCM-manifold: a two-dimensional torus, to represent the actions, whereas it is projected to a temporal axis representing the interaction with the medium. Therefore, DCM operations can be depicted as toroidal trajectories deformed by a time-dependent environment parameter $(\epsilon(t))$ and internal dynamics. First, we need to consider that in general, the dynamics on manifolds will occur at local and global scales [69]. Our mechanism of structural coupling, DCM, will thus bring forth phenomena belonging to these different scales. The operation of DCM at local scales leads to biological phenomenology related to ontogenic processes; whereas in the global scale dynamics shapes phylogenetic processes. Second, Biological Organization operates continuously by decomposing the structure of the perturbations $\epsilon(t){ }^{14}$ associated to the dynamics of the physical medium. DCM decomposes $\epsilon(t)$ in the construction of $\mathcal{H}^{\mathcal{B}}$

\footnotetext{
${ }^{13}$ for every physical system there is a function defined on its space of states called the energy or Hamiltonian of the system containing all its dynamical information.

14 also understood as frequency interference; perturbation series, frequency content perturbation, etc..
} 
while simultaneously projecting $\epsilon(t)$ into local and global components of dynamics. DCM-manifold analysis, with the underlying KAM dynamics, is obtained by the standard methods of the phase space analysis, including numerical and Poincaré sections (PS) analysis for system's dynamics.

In general, KAM dynamics contains three main regimes of mathematical-actions: the stable KAM surfaces; the asymptotic-Nekhoroshev slow diffusion and the stochastic diffusional Chirikovchaotic regimes, both closely related to Arnold web/diffusion [70]. In non-biological, ideal (fully integrable) systems the unperturbed situation $(\epsilon=0)$ produces trajectories of actions lying in multiple concentric tori of well isolated energetic KAM-surfaces. The PS, in this case, is filled with well defined circular sections of periodic actions (orbits). In non-ideal physical systems $(\epsilon \approx 0)$, quasi-periodic actions become deformed (expanding or contracting) towards adjacent energetic KAM-surfaces and the PS become discontinued in a dense circle [71]. The case, for $0<\epsilon<<\epsilon_{c}$, where $\epsilon_{c}$ is a critical value, the resulting dynamic makes continuous transitions between ideal to real conditions; i.e. from periodic to quasi-periodic actions forming the KAM-surfaces. The KAMsurfaces contains continuous smooth trajectories reaching distant points in the phase space. The PS appears as closed orbits containing concentric KAM-surfaces they are deformed ellipsoids; the diffusion is confined inside, and trajectories do not cross the KAM-surfaces. The travel-time $(\tau)$ between distant points in phase space depends on the trajectory-length and the magnitude of $\epsilon$. Near KAM-surfaces, trajectories are asymptotic and for small perturbations, $\tau$ is very large. The largest Lyapunov index $(\lambda)$ is negative and $\tau$ is in the Nekhoroshev range $\left(\mathcal{O}\left[\exp \left(-\epsilon_{c} / \epsilon\right)^{c}\right]\right)$. Far away of KAM-surfaces, the trajectories are stochastic and $\tau$ is proportional to $\epsilon$. For biological systems, a fundamental difference exists. Biological Hamiltonians $\left(\mathcal{H}^{\mathcal{B}}\right)$ acquire additional structure due to System $\leftrightarrow$ Medium interactions (i.e. $\epsilon(t) \neq 0$ ) and thus continuously incorporates, via DCM and recurrent history of interactions, regularities of the medium into $\mathcal{H}^{\mathcal{B}}$. This fundamental plastic property does not exist for Hamiltonians describing non-living physical systems. Two consequences are immediate; first, $\mathcal{H}_{\epsilon(t)}^{\mathcal{B}}$ defines a history of recurrent interactions System $\leftrightarrow$ Medium and it re-enacts the niche (in the sense of Von Uexkull's Umwelt) as well as recreating the niche condition of existence. Second, as to know is to act, DCM generates a mechanically based cognition in parallel with niche creation.

In summary: the system directs the history of interactions with its medium, by the structure of the functional symmetries (which continuously implement the physical actions of the system) and, via DCM, incorporates this history in $\mathcal{H}_{\epsilon\left(t_{0}\right)}^{\mathcal{B}}$ as an special diffusion-evaluation of its inter- 
acting network of functional symmetries. Importantly, DCM underlies the basic tenet of Biology of Cognition; "knowing is doing", and thus "behavior" produces coherent actions in congruence with the medium and circumstances. In the next section we will discuss how cognition and living organization are intimately related and cannot be separated.

\subsubsection{Cognitive and Environmental Relations in BOP}

For Biology of Cognition, cognition is understood in terms of the concrete flow of interactions between organisms and their medium resulting in consistent actions allowing the conservation of the organism's organization. This conservation appears, for external observers, as adaptive behaviors confronting external circumstances. The operation of living systems as cognitive devices in the BOP framework sheds light on how organism-medium interactions are established and stabilized. Crucially, under DCM, living systems operate as Bayesian inferential machines.

Biologicaly Organized Cognitive Machines. At the core of BOP, the structure-function relationships operated by DCM, establishes morphometric quantities which associate to the functional symmetries supporting $\mathcal{H}_{\epsilon(t)}^{\mathcal{B}}$. A morphometric quantity relates its particular physical domain of structure-function to the organizational space where it becomes associated with a set of functional symmetries which entail it. Morphometric quantities are quite variable as they depend on the physical context where they exist and, more importantly, on how the underlying set of functional symmetries (defining them) participates in defining $\mathcal{H}_{\epsilon(t)}^{\mathcal{B}}$. Examples of morphometric quantities are: in chemical reaction networks, the efficiency of enzymatic catalysis ; in excitability networks, the open-probability of ion-channels or the synaptic efficiency of neuronal networks (producing Hebbian plasticity or the newly recognized meta-plasticity mechanism).

The system-medium interactions, which are reflected in the parameter $\epsilon(t)$, engages ensembles of functional symmetries which propagate a diffusive wave of perturbations. This perturbation wave occurs in the Biological Organizationspace and then we called it Organizational Wave. When the functional symmetries are projected in the physical space, their associated morphometric quantities, perform a wave of interactions held by their physical properties. Therefore, the morphometric quantities which underpin the organizational waves are assembled by the recurrent operation of DCM. As DCM constructs an increasingly smoother organizational wave propagation at the same time of also forming the nearly integrable $\mathcal{H}_{\epsilon(t)}^{\mathcal{B}}$. The net result is that $\epsilon(t)$ reflects both the smoothness and the closure (quasi-integrability) of the organizational Hamiltonian $\mathcal{H}_{\epsilon(t)}^{\mathcal{B}}$. The recurrent 
system-medium interactions progressively become smoother resulting in progressive changes in the set of morphometric quantities physical properties. Then, the network of functional symmetries underlying the $\mathcal{H}_{\epsilon(t)}^{\mathcal{B}}$ at different spatio-temporal scales become adjusted by the corresponding changes in morphometric quantities. The changes in morphometric quantities occur as the differential use in propagating smother, stronger and robust organizational waves. Hence the particularly engaged ensemble of morphometric quantities is systemically defined and used continuously as behaviouralactions resulting from $\mathcal{H}_{\epsilon(t)}^{\mathcal{B}}$ into a cognitive device implementing Bayesian inference.

Therefore, a Bayesian mechanism arises by the operation of the Forward and Backward ChapmanKolmogorov equations that implement the core of DCM. When system-environment interacts, it generates a set of parallel organizational waves that are underpinned by parallel waves of activated morphometric quantities. The properties of these waves (both organizational and physical) define a flux of interactions defining the recurrent interactions with the medium. As the DCM operation produces smother propagation of the waves, the flow becomes more compact and correlated reflected in changes in the divergent (Kullback-Leibler divergence) of the associated flows and lower relative entropy. The result is that the properties of the propagating waves form dynamical spaces (manifolds) of quasi-integrable trajectories, because of the recurrent operation of DCM, become closer to KAM-surfaces, starting from random or chaotic regimes among interacting functional symmetries. Then, the resulting waves in the interaction networks of functional symmetries parallels morphometric correlations guided by the quasi-integrable trajectories in the KAM-surfaces. The increment on morphometric correlations as successive cycles of system-medium interactions leads to establish morphometric computations that improves their correlations as $\mathcal{H}_{\epsilon(t)}^{\mathcal{B}}$ become smoother. As system-medium interactions lead to smoother trajectories in each cycle of recurrent interaction, $\epsilon(t)$ is computed by the system organization as well as by the morphometric computations. In this way, massive-parallel computations of $\epsilon(t)$ are performed by the speed and amplitude of the perturbation wave, as well as reflecting the physical coherency of the morphometric ensemble. In this way, parallel computations, are at the central core of DCM, and map $\epsilon(t)$ to $\mathcal{H}_{\epsilon(t)}^{\mathcal{B}}$ at the organizational level as a historical process of coherency system-environment actions underpinning Cognition.

Interestingly, $\epsilon(t)$, in the context of the constraints imposed by the KAM-theorem leads to two important consequences: first, KAM-theorem warrants the proper computation of $\epsilon(t)$ as a condition of existence to preserve Biological Organization invariant. Second, the conditions needed to satisfy KAM-theorem (like the irrational frequencies) allows the morphometric computations to 
be broadly distributed in as many parallel assemblies as frequencies content of $\epsilon(t)$. Similarly, the relation with cognition arises from the capability of decomposing analytically $\epsilon(t)$, via stochastic diffusion implemented in DCM. Notably, as DCM is a universal operation, $\epsilon(t)$ is traced also as a historical process defining the system-niche duality. The coherency of $\epsilon(t)$ computations across different systems' organizational lineages extracts features of the shared system-medium interactions and defines a macro-organization as an integrated ecosystem that further develops as an organism and the related modes of living enabling Anticipatory Computing in all the integrated scales.

In summary, recurrent perturbations on the biological structures, associated to the functional symmetries, drift $\mathcal{H}_{\epsilon(t)}^{\mathcal{B}}$ and forcing it to follow the constraints imposed by KAM-controlled fluxes which define the biological organization as well as behavioral-actions. Thus $\epsilon(t)$ is computed and included as smooth drifts in the organization which then reflects the regularities of the external milieu by the mapping, $\Psi: \epsilon(t) \stackrel{\text { G }}{\mapsto} \mathcal{H}_{\epsilon(t)}^{\mathcal{B}}$ and which complies with the following differential equation

$$
\frac{\partial}{\partial t} \Psi\left(\epsilon(t), \mathcal{H}_{\epsilon\left(t_{0}\right)}^{\mathcal{B}}, t\right)=\mathcal{H}_{\epsilon(t)}^{\mathcal{B}}(\Psi(t))
$$

and solved as

$$
\Psi\left(\epsilon(t), \mathcal{H}_{\epsilon\left(t_{0}\right)}^{\mathcal{B}}, t\right)=\exp \left(t \mathcal{H}_{\epsilon(t)}^{\mathcal{B}}\right)\left(\Psi\left(t_{0}\right)\right)
$$

Thus the Hamiltonian representing living systems $\mathcal{H}_{\epsilon(t)}^{\mathcal{B}}$ is defined by a complex expectationdrifting process (asymptotic stochastic integral), included in the algorithmic operator which incorporates, in its definition, sample-path integrals and the FKCE and BKCE; taking also into account internal $\left(\mathcal{H}_{\epsilon\left(t_{0}\right)}^{\mathcal{B}}\right)$ as well as external perturbations $(\epsilon(t) \circledast H(I, \theta))$ to produce the correct setting for the KAM-theorem. This self-reference mechanism makes the system to behave, to external observers, like a cognitive device using its experience to tune its behaviors by continuously allowing directed adaptive structural changes in the coherence (covariance) of the morphometric quantities that extremizing $\Psi$. The results of DCM recurrent operations on the biological Hamiltonian implement a Biologicaly Organized Cognitive Machines (BOCM), capable of cognitively evaluatedecide-act upon environmental changes in a coherent adaptive way; while keeping invariant the basic operations defining BOCM itself.

The operation of BOCM can be compared to the standard model of computation embodied by Turing Machines (TM). To understand the crucial differences between BOCM and TM we need first to summarize the main aspects of TM operations: They operate on a string of symbols 
that are algorithmically transformed, the meaning of the symbols, as well as the internal rules of transformation (program), are externally defined. TM execute their program by following a sequence of Read-Evaluation-Print and Loop (REPL), this sequence can stop or not. In some cases, which in general are impossible to deduce, the machine reaches a special state (HALT) and stops further REPL evaluation. Then the output of the computation is well defined and can be read in the output string. Also, in the theory of TM, they exist hidden entities that while not being a part of the theorems they are central: the users and the programmers. Finally, TM can be implemented in different materials and contexts ranging from transistors to gene regulatory circuits. The syntactic and semantic content of the manipulated symbols must be constructed and shared by users and programmers and this shared creation of meaning is a complementary process performed outside the TM mechanism. TM and BOCM are different mechanisms and we focus on the underlying dynamics of both types of machines to illuminate their main differences.

In the case of TM, the internal dynamics are quite simple, while BOCM posses complex internal dynamics reflecting the time-evolution of a physical system following trajectories subjected to minimization. BOCM first operates using morphometric computations to assets the structure of the medium, via estimation of $\epsilon(t)$, through generating behavioural-actions in correspondence to changes in the medium. Then, BOCM operates over mixed modes of coding/decoding schemes represented by a heterogeneous set of morphometric quantities which are dependent on the particular assemblies of functional symmetries operating. Binary functional symmetries, implements a binary coding/decoding schemes, whereas multinomial functional symmetries represent multi-modal coding/decoding schemes.

Biological computations are fundamentally different from computing in Turing systems. In TM, the initial and final states are perfectly defined in the device. By reaching the halt state, the algorithmic computation is finished, making the output accessible for external readers. On the other hand, BOCM computations differ as the initial and final states are not defined for the device or instantiation of DCM. No halting is ever reached as the BOCM always continues in a never-ending flow of interactions with its medium. These computing associated flows use morphometric interactions/evaluations, which correspond to the extraction of multidimensional covariant-correlations of sensor-effector activities. These multidimensional extractions should be viewed as asymptotic BOCM-generated biological computations. Then $\Psi$ encodes the medium and the adaptive coherent actions emerging from the continuous flows in Hamiltonian dynamics. The interaction between 
assemblies of functional symmetries and associated morphometric quantities entailed in structural coupling and the phenomenon of cognition. In this way, sensor-motor coherences entails adaptive interactions with the medium, implementing operational closure. $\Psi$ simultaneously entails anticipation and knowledge of its extended niche, the physical and biological medium (ecosystem). The above operation of DCM defines a architecture and operating system as well as cycling for a parallel universal machine that uses morphometric quantities as calculating elements analogous to conventional bits (i.e. b-bits), in the state variables of functional symmetries. In this context information is embedded and distributed in the structure and organization (i.e. the actions of morphometric quantities) contained in the living system and the flux trajectories in $\mathcal{H}_{\epsilon(t)}^{\mathcal{B}}$.

Therefore, BOCM define their own hardware and software through their interactions with the medium and the niche they define and operate. These machines have -from binary to quantum-like - hybrid processing capabilities. Embodied in the states of functional symmetries, from binary to multinomial, they are structurally coupled to the environment. Notably BOCM self-programming exploits diffusional Bayesian Inference over DCM-organized fluxes of least-actions. BOCM, via DCM, estimate $\epsilon(t)$ through sensory-effector loops, produced in the fluxes of least-action by metaplasticity which estimates the Kullback-Leibler divergences of those fluxes (Entropy Estimates). Programming in BOCM is made by self-reference (self-programming) without making special requirements to inputs nor outputs. Instead of programming by writing rules (representing logical operations) BOCM are programmed by the parallel and continuous operation of DCM, which evaluates asymptotically a multi-dimensional representation of the medium by the structure of $\epsilon(t)$. Importantly, the parallel operation of DCM at different hierarchies and sub-systems constructs specific $\mathcal{H}_{\epsilon(t)}^{\mathcal{B}}$, each one representing a Hamiltonian embedding. These embeddings parallelisms of DCM operation implements recursive probabilistic inference estimations on Bayesian networks using the functional symmetries' interaction networks present in the embeddings.

Similarly, DCM leads to implementing template-copy type procedures as they are a mechanism of structural coherence formation and occur in many instances in biology. Moreover, this property of DCM relates to the coherence extraction-formation as a way of Organizational Encoding reflected in the widespread copy over template procedures found in biology and finally in reproduction as a key process in Biological Closure. BOCM, perform recursive computations over $\epsilon(t)$ and the history of interactions recorded in $\mathcal{H}_{\epsilon(t)}^{\mathcal{B}}$, implementing Cognition, self-programming and self-reference, creating subjective meaning shared by similar living systems. This collective creation of meaning 
is the mechanism at the base of Biosemiotics [72, 73]. Current implementations of modern machine learning, as done in deep learning-AI, approaches DCM core process as they extract higher-order coherences implicit in the training datasets leading to predictions as a continuous refinement of extracted \& predicted patterns. More importantly, BOCM spontaneously self-replicate as the result of the recursive computations of the organizational architecture implied in DCM operation. This organizational computation architecture (self-programming, and self-replication of software and hardware in embedding operation of DCM) evaluates the continuous computations resulting in the biological Hamiltonian $\mathcal{H}_{\epsilon(t)}^{\mathcal{B}}$ and its cognitive behavior through action on the medium. Overall, these characteristics make BOCM to act as cognitive devices where behavior is modified by the experience to keep the Organizational Architecture as a geometrical invariant of $\mathcal{H}_{\epsilon(t)}^{\mathcal{B}}$.

Niche definition in Symplectic DCM-Manifolds. As mentioned two basics characteristics of DCM are self-organization resulting from the continuous construction of $\mathcal{H}_{\epsilon\left(t_{0}\right)}^{\mathcal{B}}$ and the incorporation of the history of system-environment interactions on its organization lineage. We now consider how the definition of niche creates local and global dynamics scales in DCM-Manifolds. In terms of the associated dynamics, a local scale of interaction with the medium is defined by cognitive algorithms implemented by DCM operation. In this local scale, fast diffusion-evaluations in the configuration and phase space are coupled in fast time scales by recurrences contained in $\epsilon(t)$ and the mathematical-actions trajectories. The structurally coupled functional symmetries and sensory-motor correlations are deployed as behavioral plasticity. In other words, the environmental perturbations, $\epsilon(t)$, induce a circulating wave, in the space of functional symmetries, spreading over DCM-manifolds as the mathematical-actions trajectories in $\mathcal{H}_{\epsilon(t)}^{\mathcal{B}}$ of the associated Hamiltonian dynamics. In phase space, DCM operation appears as a diffusive excursion of the Hamiltonian trajectories over the network of functional symmetries supporting the local dynamics. The perturbations are continuously computed and refined by the parallel recursive computing architecture implicit in BOCM operations. These fast-time and local dynamics represent quasi-integrable Hamiltonian dynamics in local symplectic manifolds. The position-momentum, area-preserving structures in phase space for Hamiltonian dynamics of mechanical systems. For $\mathcal{H}_{\epsilon(t)}^{\mathcal{B}}$, the symplectic structures preserves the organizational features (dynamical order) invariant as well as the Structural Coupling which assembles the fluxes of the functional symmetries connected by DCM in a cognitive niche definition. 
On the other hand, global dynamics are established by incorporating structural changes in the structure-function relationship of the underlying network of functional symmetries encompassing slower time scales. The perturbations contained in $\epsilon(t)$ and the coherence between morphometric features become modified in association to the propagating-wave solving $\mathcal{H}_{\epsilon(t)}^{\mathcal{B}}$ in the continuous context of the system-environment interactions. As morphometric quantities relate to functional symmetries and, in turn, covariates with structure-function relationships; coherences increase between morphometric features and the perturbations leading to more robust propagating-waves of functional activation across DCM-manifolds. These propagating-waves form quasi-integrable trajectories of mathematical-actions defining $\mathcal{H}_{\epsilon(t)}^{\mathcal{B}}$. Thus, changes in the morphometric quantities coherence become associated with enhanced and smoother $\mathcal{H}_{\epsilon(t)}^{\mathcal{B}}$ in symplectic manifolds. As the changes in coherence in morphometric features are also associated with changes, constraints, in the structure-function relationship, and then they lead to slow changes in ontogenic and phylogenic time scales associated with intermediate and global scales of $\mathcal{H}_{\epsilon(t)}^{\mathcal{B}}$ embeddings.

In summary, to have an understanding of organisms it is necessary to include its niche as well as its history of interactions as integral parts of the definition of the organism. In the BOP framework, the operations in DCM actively produce the organism's niche while recursively forming $\mathcal{H}_{\epsilon(t)}^{\mathcal{B}}$. Thus the structure of the external medium is not objectively defined per-se as it is the result of a process where organism and medium became actively entangled and thus inseparable. The niche is not the mere space of existence of an organism filtered by its sensorium; instead, it is the self-organized space of existence resulting from plastic and mechanical actions entailed by the continuous operation of DCM due to Biological Closure and Structural Coupling. The result is the formation of a historical organization lineage in $\mathcal{H}_{\epsilon\left(t_{0}\right)}^{\mathcal{B}}$, in which internal and external perturbations are involved as well as local, medium and global scale dynamics of DCM-manifolds. Therefore, phylogeny and ontogeny as well as developmental and physiological processes, become part of the organizational $\mathcal{H}_{\epsilon(t)}^{\mathcal{B}}$ defining the extended niche and the biological lineage representing a history of morphometric changes coupled to $\epsilon(t)$. Our analysis describes a central and universal mechanism (DCM) for living systems and the resulting dynamics (KAM-theory) by which organisms, their niches (including the medium) and the history of interactions between them form an integrated (entangled) system-niche duality. These features cover several scales of organizational Hamiltonian dynamics on self-constructed Symplectic Manifolds. 


\subsubsection{Origin and Evolution from BOP}

We would like to address two main aspects related to the evolution of living systems as they have not been addressed by other theoretical frameworks.

Biological Origin via DCM. Our framework proposes DCM to be the basic mechanistic core of the biological organization and also it serves to understand the initial, and essential, steps of biogenesis. Biogenesis achieves a primitive implementation of DCM by turbulent coupling of simple organic reactions. In the primitive geochemical conditions, simple chemical reactions occur spontaneously as they dissipate (electro-) chemical gradients. These primitive chemical networks likely had laminar diffusion in some places; whereas in other places can be dominated by inhomogeneous and turbulent mixing. Furthermore, a basic set of mass-action-favored reactions proceed towards steady-state conditions following the limited-diffusion laminar fluxes. Thus simple chemical networks transiting towards equilibrium can become a pseudo-metabolism once a sufficient degree of Biological Closure, created by DCM, is achieved. Each transformation reaction scheme as in (7), is characterized by its kinetic transformation constants $\left(\alpha_{i} ; \beta_{i}\right)$. These forward and backward constants constitute the most primitive instances of a morphometric quantities. While the set $X_{i} \rightleftarrows\left(1-X_{i}\right)$ which are the components of the pseudo-metabolic network proceed towards steady states equilibrium they are also coupled by sharing some reactants, products, and catalyzers. DCM, in this condition of shared components and coupled reactions, improves the coherence (correlations) between the sets $\alpha_{i}$ and $\beta_{i}$ across the network. The correlation improvements can be achieved by various paths. One is through variations in the structure of the catalyzers, as for example the ones increasing the efficiency of a reaction. Another can be made by forming a new species that, because of its participation in new reactions, decrease the network pathways and thus enhancing the co-variation between concentrations of different chemicals. This last procedure relates to changes in the deficiency of the chemical reaction networks [74, 75, 76], whereas the first relates to the thermodynamical activation and kinetic (dynamics) features of the pseudo-metabolism. In the primitive geochemical environment pseudo-metabolisms, made by small finite numbers of detailed balance transformations (as 7) each of which represents a functional symmetry, form ensembles of morphometric quantities (as sets of $\alpha_{i} ; \beta_{i} ; X_{i}$ for binary symmetries) under DCM-controlled co-variation. DCM explores the stochastic couplings among the set of reactions and implements the continuous refinement of $\mathcal{H}_{\epsilon(t)}^{\mathcal{B}}$. The perturbations associated with turbulent fluctuations become incorporated in $\epsilon(t)$ and in the 
history of perturbations impinging into the pseudo-metabolisms (constrained by limited-diffusion in laminar fluxes). Then, DCM produces coherency between the morphometric quantities starting a primitive $\mathcal{H}_{\epsilon(0)}^{\mathcal{B}}$ at the biogenesis of pseudo-metabolism, which is at the ancestral origin of the lineage leading to the autopoietic organization. Note that the primitive Hamiltonian describing the system entails that the chemical reaction network must contain (quasi-) closed direct graphs. This primitive pseudo-metabolic organization, which operates under DCM, however, it is not an autopoietic entity as is not been encapsulated yet. Successive recurrence between the turbulence containing pseudo-metabolisms and the progressive addition of others electro-chemical dissipating networks end forming coupled Hamiltonian of increasing complexity in a mixed structure of port-Hamiltonians $[63$, 77, 78, 79]. As further environmental fluctuations occurs, as was explained previously, inclusion of newer variational structure in the context of KAM-theory is performed and thus increases in the complexity of $\mathcal{H}_{\epsilon(0)}^{\mathcal{B}}$ is achieved in a coherent evolution lineage.

In summary, the DCM core implementation of our framework is simple enough to be highly likely to have occurred in the primitive geochemical conditions when turbulent fluxes contained basic organic chemical reactions, as for example hydrothermal vents. Notably, our scheme does not depend on the properties of special kinds of molecules (like DNA o RNA) rather it depends on the collective properties of coupled networks constrained by DCM. The spontaneous generation of Hamiltonians describing early chemical reaction networks under limited diffusion conditions: a) increases the likelihood of encapsulation and b) increases the appearance of new organizational proto-metabolic evolutionary lineages; these two distinctive features of BOP are important for the origin of living systems. Furthermore, these distinctive features are entailed in DCM, which sharpens molecular mechanisms of the living organization (either in proto metabolisms as well as in autopoietic systems) while incorporating environmental fluctuations into its organizational dynamics. It is important to realize that, from our framework viewpoint, the primitive organization exists as a dynamical entity enclosed by limited-diffusion without requiring a semi-permeable lipophilic membrane or micelles. Moreover, a soft or primitive autopoietic metabolism (pseudo-metabolism) can be constrained in a limited-diffusive ensemble of steady-state chemical reactions not necessarily organically-catalyzed.

Natural Drift Evolution via BOP. The recursive nature of DCM operations leads to the formation of metabolic lineages which evolve by incorporating in their organization the interactions with the 
medium. The evolution trajectory is continuously shaped by DCM and constrained by KAMtheoretical dynamics. The living organization, through DCM, is subjected to local and global dynamic constraints that channels the structural changes; in which parallel dimensions of ontological (local) and phylogenetical (global) changes coexist.

DCM forms organizational diffusional waves that allow for the continued exploration of phase spaces of the complex geometry entailed in the continual operation of DCM. Thus the geometric entailment of the biological Hamiltonians $\mathcal{H}_{\epsilon(t)}^{\mathcal{B}}$, drifts the associated organization towards near KAM-surfaces containing particular dynamics. Therefore, DCM allows the evolution dynamics of an organization to continuously drift accordingly to the history of interaction and the recurrences with the medium as an asymptotic expectation. Thus, KAM-theoretical dynamics underlie the evolutionary trajectories and the associated morphological changes; as the associated evolving biological organization always must comply with the dynamics deriving from KAM-theorem and implied in $\mathcal{H}_{\epsilon(t)}^{\mathcal{B}}$. Then, as it is the case for ontogeny, a phylogenetic relationship between phenotype and niche is also intimately entailed in Biological Organization Principles. Then, BOP conveys a formal version of Evolution by Natural Drift [80. In BOP, the continuity of phylogenetic lineages implies that different ontogenic-phenotypes lineages follow differently, but related, complex trajectories along KAM-surfaces. Importantly in the context of our framework, the perturbations -internal as well as external- modifies the KAM-surfaces in a very precise way associating the specific timeevolution of the trajectories to the perturbations' history. Then, the evolution trajectories are not defined only by their initial state, or by optimizing fitness but by the history of interactions with the medium, represented by $\epsilon(t)$. Nevertheless, all the possible trajectories lie in KAM-surfaces a fact that is not trivial. As we have underlined many times, DCM allows the exploration of close (near) KAM-surfaces depending on the history and intensity of the perturbations. The crucial point, to understand the origin of drift, is that once the lineage is in a given trajectory seemingly small recurrent perturbations (internal and external) can make the lineage to drift from one KAM-surfaces to another. These KAM surfaces can be underpinned by a vastly different number of functional symmetries and thus generating different biological morphologies. As the lineage drifts, new trajectories are defined by the new recursivities, the diffusion-evaluation produces a drifted, $\mathcal{H}_{\epsilon(0)}^{\mathcal{B}}+\epsilon(t) * H(I, \theta)$, which stabilizes as a new embedding in the organization using a variation (drift) of the interacting network of functional symmetries. This drifting mechanism across KAM-surfaces, a consequence of DCM, is endlessly repeated and permitted until some critical value, $\epsilon_{c}$. In effect, the existence of 
KAM-surfaces allows for a broad range of interactions with the medium until a sufficiently strong perturbation, $\epsilon>\epsilon_{c}$, destroys the KAM-torus persistence. In the regime where $\epsilon<\epsilon_{c}$ a bifurcation regime in the flux trajectories is observed $[47,53,55,81$, 82, the structure of these bifurcations, called a Cantori. Cantori structure implies lineage's trajectories diversification ensuring a huge space to organic evolution by natural drift, although with preservation of the organizational dynamics.

Globally, the system's interactions define lineages of historical recurrences in the niche definition involving lasting explorations of the phase space and changes in $\epsilon(t)$. Both of them, niche and $\epsilon(t)$ changes, occur as continuous processes of exploratory dynamics in the central manifold and the forcing actions of its associated bundles (tangent/cotangent). On the structural side, global dynamics are built by the new configurations triggered by a change in the morphometric quantities of the structure-function relationship associated with the underlying functional symmetries. Importantly, the dynamics and operation by DCM results in changes congruent with variations in the environment and thus appear as optimal with an adaptive purpose. The congruence between the living form and the environment interpreted as adaptation has been one of the intriguing, but misinterpreted, features of living forms. In this context, DCM can be understood as the basic underpinning of natural selection. Moreover, as the underlying mechanic and dynamics implemented by DCM end up with the conservative replication of the structure and dynamics of $\mathcal{H}_{\epsilon(t)}^{\mathcal{B}}$ with variations, the evolution of the organized system also become intimately related to reproduction as well as conservative variations and encoding of traits. As this process is allowed to evolve in time, the final geometry of the high dimensional Torus explored by two lineages starting from similar positions (in their phase-spaces) can greatly differ. This difference will mostly reflect the stochastic nature of the determination of $\mathcal{H}_{\epsilon(t)}^{\mathcal{B}}$ by expectations and the precise $\epsilon(t)$. Thus our mechanism of evolution exploring KAM-surfaces allows to understand evolution by natural drift in a mechanistic way and it has the advantage of making the co-existence of drift and adaptation possible and understandable in the same framework. 


\section{Discussion and Conclusions}

In this section we discuss in a broader perspective our proposal on Biological Organization Principles.

\subsection{Theoretical Key Ideas from BOP}

\subsubsection{KAM-theoretical Dynamics}

Biological Organization dynamics constrained to KAM-surfaces. As mentioned, the dynamics of the living organization always happen inside a high dimensional KAM-Tori which is difficult to visualize. The relevant core of KAM-theoretical dynamics can be already appreciated in lower-dimensional cases as it is well known for regular motion or for the classic Taylor-Chirikov Standard Map, or standard map for short. Area-preserving maps, like the standard map, give rise to rich dynamics and mathematics appearing in many applications including mechanics, chemistry, celestial dynamics, plasma physics, condensed matter physics, and many other fields. The standard map is very useful as representing the local dynamics for several dynamical systems [71, 83]. Then, KAM-theoretical dynamics can appear in this low dimensional Hamiltonian system [83]. The standard map considers two coupled variables - rotation angle and momentum $(\theta, p)$ - in the following discrete nonlinear system:

$$
\begin{aligned}
& p_{i+1}=p_{i}+K \sin \left(\theta_{i}\right), \\
& \theta_{i+1}=\theta_{i}+p_{i+1}
\end{aligned}
$$

for the $i$-iteration of the map, and the parameter $K$ "kicks" the system. This system represents a forced pendulum receiving impulses of amplitude $K$ thus explaining the colloquial name of this system as kicked rotor. For $K=0$ equation (17) shows periodic motions, for any initial condition $\left(\theta_{0} ; p_{0}\right)$, as the momentum does not change $\left(p_{i}=p_{0}\right)$ thus filling phase space ( $p$ vs $\theta$, constant $\left.=p_{0}\right)$ with parallel trajectories of regular motion. Regular motion for the case of the forced oscillator pendulum relates to the smooth motion describing the periodic movement around a reference point (for example the central axis) $(\theta=0)$ of the resting state. As $K$ increases $(>0)$, the momentum is modulated proportional to $K$, and some trajectories become irregular motions, whereas others are still regular in the regime for some initial conditions as it is guaranteed by KAM-theorem $\left(K \leq K_{c}\right)$. For even larger $K$ fixed points appear as well as subsets of trajectories where motion is constrained. As $K$ increases even further, the trajectories exhibit deep modulation and the subsets 
of constrained motions (a KAM-torus) continue to exist. In these subsets of trajectories, orbits remain continuously bounded and KAM theorem guarantees, up to some perturbation level, the existence of these subsets where motion remains constrained. As $K$ progressively increases, chaotic diffusion appears and becomes delimited by KAM-surfaces. KAM-surfaces establishes asymptotic surfaces of periodic and quasi-periodic motions. The process of disintegration of these surfaces, which blocks the generalized chaotic (linear) diffusion, is a KAM-theory cornerstone. After a critical value $\left(K_{c}\right.$ that for this map is near 0.9 ), the last KAM-surface is destroyed and chaotic diffusion appears everywhere.

We will use the standard-map, even when it is a low-dimensional nonlinear manifold, as a model example on how BOP can be understood in the context of KAM-theoretical dynamics. Despite its simplicity as a low dimensional example, the standard-map formalism captures the core operation of the biological ideas of Biological Closure and Structural Coupling (BC\&SC). It is interesting to note how the duality organization-niche is formed and shaped by DCM. The standard-map represents a closed motion trajectory that results in the periodic movement of the object-body position. In the case of BOP, the functional symmetries are represented as systemic phasors with $\theta$-angle oscillating pendulums. In the standard-map, $\theta$ and $p$ can be considered the position and momentum of a pendulum with an impulsive torque $(K \sin (\theta))$ at the end of the pendulum. $K$ represents the amplitude of the "kicks" and determines the extent of the nonlinearity of the system. Likewise, the system's surroundings will be associated with the interactions with the fundamental elements in BOP, the functional symmetries - which for the case of the standard-map occurs by $\sin (\theta)-K$ coupling. A system organized along these lines will exhibit the phenomenon of cognition arising from the duality organization-niche build by DCM. Finally, we will indicate how the different lineages of organizational-niche duality must be understood as deriving from the constrained KAM-theoretical dynamics associated with the standard-map. We will use the standard-map as a model example on how BOP can be understood in the context of KAM-theoretical dynamics 15 . Despite its simplicity as a low dimensional example, the standard-map captures the core operations of the biological ideas of Biological Closure and Structural Coupling (BC\&SC). It is interesting to note how the duality organization-niche is formed and shaped by DCM. The standard-map represents a closed

\footnotetext{
${ }^{15}$ Although the standard-map is a low-dimensional nonlinear manifold higher dimensional generalizations, the generalized standard -map [84, 85].
} 
trajectory motion that results in the periodic movement of the object-body position. In the case of BOP, we represent functional symmetries as systemic phasors; $\theta$-angle oscillating pendulums. In the standard-map, $\theta$ and $p$ can be considered the position and momentum of a pendulum with an impulsive torque $K \sin (\theta)$ at the end of the pendulum. $K$ represents the amplitude of the "kicks" and determines the extent of the nonlinearity of the system. Let us consider the standard-map or kicked rotor as representing the deterministic behavior of the system resulting from DCM operation. The organized system-niche duality emerges from a stochastic background following the formation of $\mathcal{H}_{s t}^{\mathcal{B}}$. The disintegration of the quasi-integrable Hamiltonian via KAM-theorem is exactly reversed by plastic changes in the assembly of functional symmetries in the chain propagating the organizational waves-oscillations as is represented in the standard-map. We should underlie that DCM is a continuous operation underpinning BC\&SC. Functional symmetries are abstracted, in the standard-map, by the rotation angle and angular momentum. We suppose that the system has already constructed a smooth $\mathcal{H}_{d t}^{\mathcal{B}}$ in the form of the oscillation, in this simplified model we will analyze how Biological Closure and Structural Coupling are continuously maintained and expanded towards new system-environment interactions 16 .

Biological Closure in this simplified model is represented in several facets. i) the integrable Hamiltonian at $K=0$; in these conditions, $p_{n}=p_{0}$ and $\theta_{n}=\theta_{0}+n P_{0}$. All the behavior of the system is specified by the initial momentum (impulse) and the initial release angle, $\theta$. If the momentum is rational with $p_{0}=M / N$ for some $M, N$ integers, then the trajectory is a period-N orbit. The trajectory orbits are quasi-periodic and densely fill in the torus for all $\theta$. The phase space contains parallel lines of regular motions. $\mathcal{H}_{t_{0}}^{\mathcal{B}}$, where periodic motion is specified $(\bmod 2 \pi)$; ii) the rotation angle, $\theta$, is periodic $(\in[0,1])$ and iii) the rate equation in $(7)$, is systemically modulated by the kicked rotor momentum as $\theta_{i+1}=\theta_{i}+p_{i}+K \sin \left(\theta_{i}\right)$. While Structural Coupling appears in; iv) the systemic dependencies $(\sin (\theta))$ on the dynamical structure of the system $(\theta)$ and $\mathrm{v}$ ) the perturbations strength $K$. Thus DCM acts by orchestrating points i to v; however, in these low dimensional conditions, some features are reduced to a single parameter, especially for the perturbation/coupling with the medium. In these conditions, $K$ is actively constrained

\footnotetext{
${ }^{16}$ At this point, when stochastic interactions already have formed a system which displays a smooth $\left(\mathcal{H}_{d t}^{\mathcal{B}}\right)$ and biological closure, as well as structural coupling, have been implemented in a structure and functional relationship in a "deterministic" instantiation. In this expansion, $\mathcal{H}_{d t}^{\mathcal{B}}$ will incorporate the organizational changes on its own structural definition as it is also a reflection of the new interactions system-environment.
} 
to be less than $K_{c}$, the critical value destroying KAM-surfaces on phase space. The constraint mechanism is the result of plastic changes affecting the angle dependency, $\sin (\theta)$. These changes can be accomplished as an angle resetting mechanism that becomes part of the structure of the initial rotor giving rise to a rotor lineage: note that the simplest mechanism is generating diffusive resistance or friction in the rotor. Then, we can visualize the organization dynamics in a transformed toroidal manifold; the phase space dissected as in the standard map's PS for different values of $K$.

In a more detailed description, lets first distinguish and specify the dimensions for our model as a $3 \mathrm{D}$ Cartesian space $(x, y, z)$. We use the $x$-axis to run $K(t)$ which is constrained by DCM to be $K(t)<K_{c}$ for $t \in[0, \infty]$, whereas the $(y, z)$-plane representing $(\theta, p)$ as in the typical PS for the standard map. We recall that in this representation, $K(t)=\epsilon(t)$ is the time evolution on the kicking strength and represents the time evolution of environmental changes. Then, the dimension along the $K(t)$-x-axis represents an instantaneous image of a fourth-dimensional time-evolving torus. This evolving torus represents a full space of systems/organization-niche dualities given the environmental changes in $K(t)$. The environmental fluctuation can be decomposed by the Fourier's frequency content, which informs the periodic changes on $K(t)$, incorporated in the structure of the KAMconstrained rotor systems. The high frequency content in $K(t)$ leads to fast ontological changes such as behavioral ones associated to learning and memory, immune responses, and physiological responses. Whereas that low frequency may lead to phylogenetic changes, associated to gene mutations and ecological "adaptations". All changes, in ontological and phylogenetic directions, involve changes in the structure-function relationship which convey also coherent modification in morphometric quantities associated to the underlying functional symmetries. The continuous adjusting of functional symmetries via modifications of the morphometric quantities leads to trajectories closer to KAM-surfaces and longer stability as well as strongest "action forces" in the phase space. Then, the local dynamical scale reflects the interactions of the underlying symmetries associated with their morphometric features. Whereas for global scale the structural modifications on the functional symmetries are stabilized by slow changes, implemented by the Biological Closure, in the correlation of structure and the actions of the organism/system behavior of the rotor lineage.

In general, local dynamics and short time scales are constrained in the phase space to local-ball domains and occur as fast stochastic implementations of DCM. Whereas, global dynamics reaches faraway locations on the phase space and PS. The action trajectories in global dynamics form the history of correlative changes in the structure underlying the functional symmetries and establish 
the phylogenetic time scales and lineages. Then, in terms of biological organization, global dynamics are very slow in the exploration of the configuration space but strong in conservative dynamics. The system's self-defined niche lies in the historical time scale of interaction with the medium and it is incorporated in $\mathcal{H}_{\epsilon\left(t_{0}\right)}^{\mathcal{B}}$. In the case of the standard map, the system-niche duality will become the combination of $\theta$ resetting mechanisms (for example a spring) and the extension of the dynamical range associated with $K$ values, which can reach values higher than the original $K_{c}$. Thus expanding the system's domain of the existence, representing the biological organization, to different values (places) in the PS while preserving dynamics (i.e. maintaining KAM-surfaces in the extended PS space) either in local and global scales as well as in short and long time scales.

In this context it is incorrect to view living systems as isolated systems that can be understood only analyzing their internal dynamics. Although it is true that internal dynamics appears defined by a robust self-reference, due to biological closure, the details of every internal process result from the stabilization of $\epsilon(t)$ through the history of recurrent interactions with the medium. Thus, as a summary of this very important point, it must be stressed that living systems are dual entities (system-niche) having an internal organization and an organism-medium interaction (ruled by DCM and the logic of BC\&SC). Similarly, this duality defines the domain of existence of the living systems as a repertoire of actions emerging from historical recurrences in system-environment interactions. Thus biological systems are, before anything else, dynamical systems constrained, by DCM, to KAM dynamics (i.e. they traverse KAM surfaces in quasi-periodic actions-motions). Nevertheless, in biological systems the situation has an added complexity as the underlying Hamiltonian $\left(\mathcal{H}_{\epsilon(t)}^{\mathcal{B}}\right)$ is the result of an asymptotic expectation of a diffusion, thus its changes at short and long time scales are due to the history of system-environment interactions which itself is the result of the previous values of $\mathcal{H}_{\epsilon(t)}^{\mathcal{B}}$. Thus a central point for any living system, besides its biological closure, is that its dynamics flow from time-dependent Hamiltonian that incorporates its own history of interactions into its organizational definition.

\subsubsection{Flux Trajectories}

Fluxes Regimes. KAM-theoretical dynamics appear in many nonlinear systems and can be understood in several mathematically complementary ways. Nevertheless, a particularly interesting view is achieved by the fluxes in phase space which associate with the interrelationship of functional symmetries and the trajectories formed by them. We will present KAM theoretical flux dynamics 
in connection with our Biological Organization Principles framework. Depending on the magnitude of $\epsilon(t)$ and of conditions derived from the system-medium interactions as well as $\mathcal{H}_{\epsilon\left(t_{0}\right)}^{\mathcal{B}}$ - the lineage duality organization-niche - different regimes in KAM dynamics develop.

Although in living systems the organizational dynamics continuously constrain $\epsilon(t)$ by (meta)plastic mechanisms, the several KAM theoretical dynamics become fully developed. Multiples KAMsurfaces form the KAM tori where quasi-periodicity occurs smoothly; stochastic diffusion between these KAM-surfaces, generating what has been termed soft chaos; and less known regimes of slow and complex diffusion as the Nekhoroshev and Arnold diffusion, respectively.

These fluxes regimes apply to living systems' organization under particular conditions of interactions system-environment. Each regime has its own implication to the dynamics of the living system, for example, in the Nekhoroshev regime slowly divergent fluxes surrounding stable KAMsurfaces appear and apply to the long-term stability of the systems. Although these are unstable trajectories, having very small divergence rates from KAM-surface they appear for practical purposes as stable fluxes.

More profound deviations from the periodicity dominated regime begin to occur at the critical value of $\epsilon_{c}$; for $\epsilon<\epsilon_{c}$ the Kolmogorov regime, the phase space contains the KAM-surfaces surrounding chaotic dynamics and the complex Arnold diffusive web. These KAM-surfaces corresponds to quasi-integrable Hamiltonian case, and represent quasi-periodic trajectories in phase space. The chaotic region between KAM-surfaces contains two main dynamical modes: close to the KAM-surfaces, a Nekhoroshev trajectory mode it is observed characterized by divergent trajectories with extremely long times of motion making the system stable for small observation times, but chaotic in the limit dynamics. In this condition the Arnold web also present complex diffusive path, representing complex drifting dynamics which connects local region of phase space with different dynamical modes. Arnold diffusion represents a very interesting topological semi-ergodic path connecting distant part of KAM-tori. Moreover, cantori formation appears in the breaking of the stable KAM-tori leading to a formidable space of action to the divergent formation of closely related organization lineages of $\mathcal{H}_{\epsilon\left(t_{0}\right)}^{\mathcal{B}}$. Finally, the Chirikov regime appears as $\epsilon$ further overpass $\epsilon_{c}$; then KAM-surfaces are destroyed and chaotic regions invade the Poincare sections with more and more regions of stochastic diffusion, Brownian-type motion.

The system's view on living organization presented here integrates key notions of modern Dynamical System Theory, the rich dynamics of quasi-integrable Hamiltonian Systems with modern 
concepts of mathematical-physics like action path integrals. The living organization then follows trajectories in phase spaces of higher dimension due that, in the organizational manifold, symplectic embedding allows for recurrent operation of KAM-theorem across organizational levels. More notable, one of the most interesting aspects is the ability of the global dynamics to support and incorporate a continuous stream of perturbations, via DCM, to establish a plastic manifold of organizational dynamics. This dynamical system implements a mechanical organization framework, represented by macroscopic Hamilton-Lagrange mechanics where KAM-dynamics is implemented.

It should be also noted that in physical systems under conservative dynamics Hamiltonians serve to understand how the energy of the system is distributed. In the biologically organized systems, DCM converts the Hamiltonian interpreted as a functional distributing energy (i.e. this is the usual interpretation of Hamilton-Jacobi equations) into an operator constraining the stochastic fields of interactions between components while incorporating perturbations into this now plastic Hamiltonians operator not only circumscribed to energy. Specifically, $\mathcal{H}_{\epsilon(t)}^{\mathcal{B}}$ manifolds in BOP contain the diffusive interaction trajectories in the phase space of the functional symmetries which, as morphometric quantities, stabilize the recurrence of interactions to establish KAM-surfaces of regular and quasi-periodic trajectories.

\subsubsection{Biological Organization Realm}

Relational interactions and living systems form. Our relational Biological Organization Principles framework is an abstraction of central organizational features of living systems. Our framework exists then in a different realm in comparison to the analysis based on shapes and forms found by observation of living organism: as others have said, the core of living systems is relational rather than structural. Our difference with previous attempts (like the Relational Biology of Rashevsky and Rosen) resides formally in producing a mechanism (DCM) which integrates several approaches including, dynamical systems, mechanical principles and non-linear dynamics of mathematical-physics. Thus our proposal greatly differs from the purely algebraic approach championed by Robert Rosen in introducing $(M, R)$-Systems. Nevertheless, the Biological Organization Principles framework constrains the structure-function relationships and how both are deployed in the physical world, as relational and structural features are the unavoidable results of the continuous modification of organization and structure governed by KAM-theorem in high-dimensional spaces. Then the morphological features observed in nature follow the organizational space properties as 
they are constrained by the underlying relational DCM dynamics. In other words, every living system and its associated morphology is in agreement with the relational evolutionary dynamics of the organizational space represented by $\mathcal{H}_{\epsilon(t)}^{\mathcal{B}}$. Therefore, the form of living systems emerges as constraints imposed by the organizational dynamics through the system-niche duality. Apparently, the form seems adapted to the niche, however, it should be better to say that morphology reflects system-niche dynamics and not its adaptation to a pre-existing niche.

Phase-space of stochastic trajectories of functional symmetries. Although the BOP framework heavily depends on some ideas from mathematical-physics, it is not mere physicalism applied to biology as profound biological notions must be used in conjunction with mathematical ideas to recover a coherent view of biological organization. Notably, functional symmetries, which are a cornerstone in our conceptualization, are notions deeply related to chemical reaction networks, microscopic equilibrium, detailed balance, and mass-action reactions kinetics. Chemical Reaction Network Theory (CRNT) lately has been extended to many other equilibrium processes using probabilistic descriptions which have been generalized in the field of stochastic mechanics, incorporating stochastic path samples, Feynman path integrals, rate and master equations and quantum mechanical techniques $[62,65]$. Importantly, the Zero Deficiency Theorem ${ }^{17}$ for metabolic flux analysis opens a topological and graph-theoretical approach to the study of steady states equilibrium in metabolic networks. Similarly, it can be used in the context of stochastic paths of functional symmetries interactions to understand in this way the biological closure properties [57, 62, 86]. In BOP, the functional features of the biological functions and physiology become abstracted into instances (metabolism, biophysics, neural fields, population dynamics, etc.) where probabilistic approaches have been validated. Basically, BOP framework serves in any situation where equilibrium distributions can be represented by probabilities density functions. In BOP probability density functions are associated with equilibrium configurations of functional states (substrate-product, active-inactive, excitationinhibition, predator-prey, etc.). Moreover in BOP, Biological Closure and Structural Coupling are added to the probabilistic view recovering topological features in the framework of stochastic mechanics to include Forward and Backward Kolmogorov equations, and their associated jump and propagation dynamics, in closed graphs of interaction networks. The fundamental result implied

\footnotetext{
${ }^{17}$ The zero deficiency theorem is a fundamental result of dynamical equilibrium and is a starting point for the study of metabolic networks $[75,74,76]$.
} 
in using these approaches is that the master equation for interaction networks is a Hamiltonian describing the evolution dynamics, i.e. probability changes, associated with the interaction of functional symmetries. Therefore, the statistical expectation, via asymptotic evaluation, of stochastic sample paths trajectories in the functional symmetries' phase-space yields closed (integrable) and quasi-integrable Hamiltonians. Although the analytic calculations of the Hamiltonian for biologically complex interaction networks are quite challenging, the mathematical-physics tools, as well as the computational methods to study these types of Hamiltonian functional, are attractive enough as they illuminate biology with a new light. Related ideas included in our framework are already being used in advanced biological research (variational Bayes, Markovian processes and Hamiltonian dynamics are already been used to study phenomena like neuronal dynamicsi [87]) we claim that a fundamental missing stage in these approaches is the absence of KAM-theory.

\subsubsection{Metaplastic Hamiltonian 83 Diffusive Organization}

Plasticity constrains trajectories to KAM-surfaces. The time-evolution of physical systems is totally determined by their Hamiltonian [57]. In biological systems, $\mathcal{H}_{\epsilon(t)}^{\mathcal{B}}$ plays such a role by endowing them with plasticity and structural coupling. In the Biological Organization space, the trajectories - which must be understood using tools of stochastic mechanics- are formed by interacting networks of functional symmetries undergoing continuous structural and relational modifications. Structural modifications are known as plasticity and meta-plasticity in the study of structure-function in experimental biology. The distinction between plasticity and meta-plasticity reflects new and important discoveries in the regulation of structural changes coming from the study of synaptic plasticity in the nervous system. These modifications change the expected Hamiltonian $\mathcal{H}_{\epsilon(t)}^{\mathcal{B}}$ and therefore induce drift in the KAM-surfaces containing the organizational evolution of the system. Furthermore, these plastic changes implement the conservative organization maintaining trajectories bounded to KAM-surfaces. The variations on the interaction networks of functional symmetries also imply correlated changes in the pathways supporting the statistical expectation and a more probably trajectory pathway. These modifications on the functional symmetries' interactions networks become slowly incorporated in the organization as the system undergoes DCM cycles. Therefore, every DCM cycle slowly adjusts the structural coupling (via changes in morphometric quantities) supporting the pathway of higher probability and shifts the expected value of the sample path integral linking each functional symmetry in the associated Markovian chain. The increase and propagation 
in the probability configuration of functional symmetries are then associated with a diffusive wave of change in the pathway of interactions, supporting the drift in organization dynamics.

Stochastic diffusion in Biological Organization. The organizational trajectories couple sets of interacting functional symmetries in a quasi-closed sequence of stochastic-sample-paths in a background of physical interactions. Functional symmetries engaged by organizational quasi-closed trajectories correspond to sequences of morphometric features supporting a stochastic diffusion wave as well as forming the Hamiltonian $\mathcal{H}_{\epsilon(t)}^{\mathcal{B}}$ through expectation. In other words, propagating waves of organizational trajectories, described by the forward and backward Chapman-Kolmogorov equations, are assembled by functional symmetries from the stochastic background of the underlying interacting networks. The forward and backward Chapman-Kolmogorov equations are central to the understanding brought forth by Biological Organization. Stochastic diffusion is fundamental phenomena in our approach as $\mathcal{H}_{\epsilon(t)}^{\mathcal{B}}$ is recursively constructed via Forward and Backward ChapmanKolmogorov equations.

Biological Organization robustness. One important consequence of BOP is the explanation for robustness (or meta-stability) of living systems. Biological robustness is the unavoidable consequence of three elements; the asymptotic integral of the stochastic path integrals (12), KAM-theorem (14) and the plastic Hamiltonian (15). The asymptotic path integral evaluation builds a quasiintegrable Hamiltonian, in the form of stochastic sample paths, from a chaotic set of interactions between functional symmetries. This asymptotic evaluation entails the stability of the systems both as a directed process and in its results (a quasi-integrable Hamiltonian) to allow the operation of KAM-theorem. KAM-theorem warrants the existence of multiple parallel Hamiltonians (integrable and quasi-integrable ones) operating without destructive interactions, as they must contain irrational frequencies and not overlapping processes subjected to nonlinear interactions. This KAM-theoretical condition is essential for the stability and robustness of simultaneous operating processes and incremental systemic complexification. Finally, the plastic Hamiltonian allows the organization to be built by dedicated mechanisms of co-variation between the physical interactions in the form of morphometric quantities. The central feature of interacting morphometric quantities is the adjustment of their structure for the stability of the functional symmetries in the organizational realm, and, in passant, the implementation of cognitive computation and adaptive structure-function relationships. In other words, for the Biological Organization the expectation 
of a quasi-integrable Hamiltonian, $\mathcal{H}_{\epsilon(t)}^{\mathcal{B}}$ constrained to KAM-dynamics must be understood as a statistical attractor towards a stable mechanical Biological Organization-tori amid a stochastic ocean of functional symmetries interaction networks. Similarly, DCM processes are fundamental to Biological Organization robustness: the plasticity of the Hamiltonian, $\mathcal{H}_{\epsilon(t)}^{\mathcal{B}}$, which continuously drifts its expectation towards a closed-integrable one and on the other hand, the systemic variations in morphometric quantities which stabilize the trajectories associated to the system-environment interactions.

\subsubsection{Biological Organization a KAM-dynamical attractor}

$\mathcal{H}_{s t}^{\mathcal{B}}$-Stochastic Biological Hamiltonian: smoothing chaos. In sections 2.3.1, 2.3 .2 and 2.3.3 we developed the central mechanistic core for the Biological Organization Principles framework. On one hand, stochastic mechanics is used to calculating the expected biological organization Hamiltonian, $\mathcal{H}^{\mathcal{B}}$, as diffusion of stochastic interactions that become stabilized by the morphometric features of the associated functional symmetries. A fundamental core concept in our model is DCM which depends on the stochastic assembly of possible trajectories enabling jump and diffusion mechanisms to operate and which are stochastic sample paths integrals in a probability landscape. This approach is general enough to be useful at the microscopic as well as the macroscopic scales enabling a probabilistic understanding of Biological Organization. The higher probability transitions shape the organizational trajectory that the system follows. When the system of stochastic sample paths interacts with the medium (via morphometric features) the interactions progressively become refined allowing structural coupling. Initially, a given system-medium interaction triggers many stochastic sample paths, reflecting unspecific relationships between these trajectories and external perturbations. If a perturbation is recurrent in time, higher probability stochastic sample paths will start to support this regular and recursive activation while a wave of activity would propagate across the dynamical assembly of functional symmetries forming sample path integrals. For each organizational level of system-medium interaction, the relevant set of engaged functional symmetries also contains a particular set of morphometric features that link the organizational and physical levels. Thus, every morphometric feature relates the structure-function relationship of the functional symmetry while directly linking the fundamental function (which resides in the organizational realm) to its structural physical features which, via Biological Closure and Structural Coupling, support biological evolution. Our BOP framework support Biological Organization dynamics in a rough 
stochastic KAM-Tori form by a stochastic Hamiltonian, $\mathcal{H}_{s t}^{\mathcal{B}}$, equation (13) which through recurrent system-medium interactions become progressively smoothed and compact (deterministic). The smoothing of the KAM-Tori courses through the statistical expectation over the sample path integrals channeling the most probable routes or trajectories of perturbations (in the system) while also defining the medium in the immediate surroundings of the Biological Organization level (i.e. the niche). In addition to these systems-medium interactions which follow a statistical trend towards classical determinism, the dynamical trajectories induced in this system-environment are constrained in stable multidimensional manifolds: the KAM-attractors. KAM-attractor theoretical dynamics are highly complex entailing deterministic trajectories with complex Cantori bifurcations forks and slow and fast diffusion webs; it also includes low levels of predictability on the trajectories and high sensitivity to system-environmental interactions as well as to initial conditions. Thus Biological Organization KAM-attractor dynamics endows the system to behave with chaotic, soft-chaotic as well as deterministic type dynamics in which the system-medium interaction have an essential role. Taken together, these dynamical properties make the organization a conservative and robust phenomena and also lie at the core of evolutionary diversity and cognitive features. In combination with morphometric quantities, they explain situated statistical modeling of the environment, phylogenetic diversity, ecosystems stability, as well as higher brain cognitive functions.

Learning and shaping the niche: smoothing expectation by concurrent sensory actions. The tight relationship between organisms and their niche has been a central element in the observation of living systems. Behaviourally, it reveals a strong connection usually interpreted as cognitive, adaptive and plastic as it continuously adjusts the organism's actions. Whereas structurally it also reveals evolutionary interactions interpreted as adaptations, traits, and ancestral interrelationships in transgenerational times. Biological Organization Principles framework proposes plasticity as a central feature common to system-niche interaction with behavioral and phylogenetic manifestations. Then plasticity is a fundamental property of living systems, not only restricted to the psychophysical or neurological manifestation of learning and memory, as it is implied in the recursive formation of $\mathcal{H}_{\epsilon(t)}^{\mathcal{B}}$ which is a key DCM process. Plasticity and learning are the reflections of the intrinsic statistical expectation procedure behind the formation of quasi-integrable Hamiltonians, and of its capabilities as a mathematical operator continuously adjusting trajectories to lie in the KAMsurfaces. Thus $\mathcal{H}_{\epsilon(t)}^{\mathcal{B}}$, the functional symmetries, and associated morphometric quantities enable 
the system-niche duality as a continuous learning process in concurrent hierarchical embeddings of plasticity mechanisms. Meta-plasticity is supported by a jump and propagation of organization activity changes mediated by the paths dependent Kolmogorov equations (PDKE), which constrains the trajectories of functional symmetries and their sample path integrals. Further assembly of many systems' components in recursive biological closure, as for example in the autopoietic state, allows systems to evolve by incorporating small perturbations (as structural/functional variability) into their organization constrained by the KAM-surfaces. This aggregation, which makes systems evolve by incorporating higher complexity, preserves the trajectories of functional symmetries implied in the conservation of the organization while allowing variability and diversity and making the systemniche duality, the predictable result in the learning of the environment and shaping of biological form.

\subsection{Back to Biology}

\subsubsection{BOP and FEP}

In the last 15 years Dr. Karl Friston and collaborators have proposed a powerful model, the FrEE ENERgy PRINCIPLE (FEP), to explain biological organization, including recasting autopoiesis, as well as adaptive behaviour using ideas derived from Statistics and Information theory (see [88, 89, 90] for early ideas and [91, 92, 93, 94] for more recent developments). The impact of FEP is prominent as it has produced a notable literature trail since its inception. Therefore, some immediate questions emerge related to the similarities and differences between the BOP and FEP approaches. First, albeit BOP is, thanks to the DCM, endowed with an intrinsic probabilistic view of biological dynamics its relation with FEP, which relies heavily on statistics-probability theory, is not immediately apparent. Second, BOP and FEP are different approaches or simply they are dual versions of each other? and third, how much they overlap in their central concepts, methods, and extensions?. A detailed answer to these questions is beyond the scope of this initial presentation of BOP, however it is important to summarize the following points.

The first similarity between FEP and BOP is that both are viewpoints embracing a wide range of phenomena associated with a formal theory of living systems embracing from bacterial metabolism to biological evolution, using advanced mathematical formalisms. While FEP formalism owes much to advanced probability theory and Bayesian inference; BOP uses ideas from mathematical-physics, in the abroad sense of mechanical and dynamical systems theory. Thus BOP's basic theoretical core 
uses tools and results from Kolmogorov's advances in dynamics and probability (KAM-theory and Chapman-Kolmogorov equations); Stochastic Mechanics (Quantum Path Integrals and Markovian networks) constituting a broader mathematical framework than FEP. In effect, FEP uses Bayesian Inference and the special construction of Markovian blankets (a type of probabilistic graphical model) as the mechanistic implementation of the probability density functions operating Bayes' theorem. Later developments of FEP extend into the mathematical-physics core, however missing KAM-theory and stochastic mechanics and path integrals as they are found in BOP. Moreover, BOP proposes a generic core mechanism (DCM) based on organizational functional symmetries, which actively constrains variation on structure-function to follow KAM-theoretical dynamics. Importantly, the evolutionary process of biological systems, as well as the ability to overcome minimal configuration states of free energy or some core element in the operation are not included in the FEP framework.

FEP central core. As we have mentioned FEP represents mainly a statistical-informational description of biological systems; for a detailed assessment of the formal ideas see [95, 96, 97. In general, FEP uses the notion of Inference in the context of maximum-entropy estimates to describe states of a system under uncertainty using the maximum-entropy principle [98, 99]. Briefly, FEP uses the following elements to represent system-environment interactions: first, the environment causes sensory responses in terms of a deterministic nonlinear function $u=g(v, \theta)$. The $u$ variable represents the sensory input activity; $v$ is a vector containing each of the underlying environmental parameters causing sensory input activation, and $\theta$ represents the parameters of a generative model accounting for the environmental effects causing the sensory activation. Therefore, the nonlinear function $g$ is the central problem of cognition to solve by the organism, and the generative model relates the causes of $v$ and parameters $\theta$, to observed inputs $u$. The generative model is constructed by posing a prior distribution over the causes $p(v ; \theta)$; that is, the probability of causes associated with the generative model for a given range of parameters. Furthermore, added to this prior distribution of causes, the generative model yields the probability distribution of the sensory inputs given the causes $p(u \mid v ; \theta)$. Then the generative model gives the probability distribution of sensory activation represented by $p(u ; \theta)$ which is computed as:

$$
p(u ; \theta)=\int p(u \mid v ; \theta) p(v ; \theta) \mathrm{d} v
$$


Then, the distribution of causes $v$ given the sensory input $u$, from the generative model parameterized by $\theta$, yields the generative conditional distribution [90]:

$$
p(v \mid u ; \theta)=\frac{p(u \mid v ; \theta) p(v ; \theta)}{p(u ; \theta)}
$$

This probabilistic description is rephrased as: lets $\vartheta$ be the environmental forces/fields acting on the (living) systems and $\lambda$ as quantities that describe the systems' physical state. Critically, it is proposed that it exists an ensemble density function $q(\vartheta ; \lambda)$ that given the medium parameters specifies the system/organism states.

"Invoking the ensemble density links the state of the system to the environment and allows us to interpret the system as a probabilistic model of the environment", [89].

Two additional variables, which are time-dependent and multidimensional, are important: $\bar{y}$ which describes the effects of the environmental variables $v$ on system states and $\alpha$ which describes the effect of the organism (system) on the environment. On a first approximation, $\bar{y}$ represents system states that are acted upon by the environment (sensory input been part) while $\alpha$ represents the action of the organism upon the environment [89]. The free energy $F$ of this setting is:

$$
F=-\int q(v) \ln \frac{p(\bar{y}, v)}{q(v)} \mathrm{d} v
$$

this definition is equivalent to:

$$
F=-\langle\ln p(\bar{y}, v)\rangle_{q}+\langle\ln q(v)\rangle_{q}
$$

This last equation can be written as:

$$
F=-\ln p(\bar{y})+D(q(v ; \lambda) \| p(v \mid \bar{y}))
$$

where $D(q \| p)=\int q \ln \frac{q}{p} d v$ is the Kullback-Leibler distance between distributions $q$ and $p$. This last equation can be re-arranged, on its first term, by taking into account $\alpha$ as:

$$
F=-\left\langle\ln p(\bar{y}(\alpha \mid v)\rangle_{q}+D(q(v ; \lambda)|| p(v \mid \bar{y}))\right.
$$

Friston gives qualitative arguments to show that any system endowed with these dynamics will expose itself selectively to the causes in the environment that it expects to encounter. The actions 
that an organism/system can perform evolves as the functions $p(\bar{y}(\alpha \mid v))$ are under continuous change.

These are the principal elements of what can be called early FEP. In this early model, the organism is only viewed as a probabilistic machine that through a procedure of optimization iteratively minimizes its free energy $F$. Lately, especially since 2011, FEP has penetrated the scientific literature with many papers exploring different aspects of the theory. One new element has been the introduction of Markov Blankets (and Markov Chains) as a tool to describe the direction of change. Still, these ideas do not endow the system with metabolism or biological processes but they begin to lift the veil on the system's internal dynamics.

The crucial step of FEP is that the organism has rules to change $p(\bar{y}(\alpha \mid v))$ to minimize $F$. This change is based on a phenomenological approach where probability distributions (in fact conditional probability distributions) get iteratively changed by following the Expectation-Maximization principle derived from Information Theory.

Thus from FEP viewpoint, an organism is a system with properties but with no internal dynamics. On the other hand, BOP does have an internal dynamics produced by DCM and which is embodied in Hamiltonian formalism. This is one of the main differences between both approaches. Two other important differences are:

- FEP lacks motion. FEP viewpoint of an organism is a system with properties but with no internal dynamics as the central idea is that an organism is an updating machine of probability distributions according to a minimization principle (free energy). Notoriously, FEP does not identify any particular material (physical) embodiment to support all the possible probability density functions which are minimized by the variational Bayes mechanism. In fact, the clearest implementation of the embodiment of probability functions is observed in neuronal dynamics which is already a complex framework (cellular and circuit levels) of activity density functions [87]. Whereas BOP begins by identifying the functional symmetries as the generalized system of coordinates as well as its higher-order derivatives. In BOP, the functional symmetries are explicitly applied to every component relevant to the dynamical construction of $\mathcal{H}_{\epsilon(t)}^{\mathcal{B}}$, identifying: enzymes, modulatory/control elements (as genes or composed biochemical processes), cells, tissues, organism, ecosystems. The mechanical approach in BOP implements the notions of Biological Closure and Structural Coupling, in the form of functional symmetries. Furthermore, BOP constructs an explicit geometrical space (sym- 
plectic manifolds) of Hamiltonians that implements Stochastic Mechanics with conservative motion. Nevertheless, the Hamiltonian perspective in BOP includes the free energy principle as it naturally follows from the dynamical fluxes implied in the Hamiltonian integrable built via DCM. In this sense, BOP always implies systemic dynamics (neural networks, chemical reaction networks, gene regulatory networks) orchestrated by DCM and which is embodied in a plastic Hamiltonian that is obtained as a drifting expectation limit. Therefore, BOP includes as a framework: mechanical, dynamical, geometric and topological aspect in addition to the expressed by FEP that is mainly probabilistic.

- KAM stability vs Expectation-Maximization minimal-landscapes. FEP, proposes the maximization of negative free energy. The net effect is that the system continuously anticipates its sensory inputs and thus makes the system work in an anticipatory mode and makes the system to always try to reach a global minimum. The use of the KAM theorem in BOP shows, not how systems fall into the local energy minimum but rather how metastability is achieved. KAM-Theory shows how a system following a particular trajectory can be perturbed to follow another set of quasi-periodic orbits. Thus under BOP approach recurrent perturbations, possessing a certain degree of regularity, can transform the dynamics of the system allowing for new trajectories. In both models fundamental functions (conditional probability distributions in FEP, Hamiltonian in BOP) are iteratively changed but the procedures performing these changes are very different. Under the FEP viewpoint, the distance between the sensory input and the internal model of the sensory input is used, using Bayesian inference, to update conditional probability distributions. In BOP the fundamental updating loop is quite different to the point that the very notion of "updating" seems a misnomer. In fact, external perturbations, with their associated probability distributions, change the internal Hamiltonian according to a diffusion process that can be computed with a path integral. In this computation, an expectation about the state of the sensory input is performed as they represent boundary states of the system.

Another interesting perspective differing BOP and FEP is on biological evolution; in the next section, we develop the BOP point of view into this central biological problem. A step-by-step comparison between FEP and BOP will demand a larger space but it will be a very rewarding 
analysis as it seems that these two viewpoints bring complementary lights into the central problem of biological organization.

\subsubsection{BOP and Biogenesis ES Evolution}

Origin. One of the hardest issues in biology is the emergence of living systems on our planet. Therefore, any relevant theory should address the basic organizational principles present in proto living systems and how their initial metabolic network became stable and robust to the point of surviving and thriving for $30 \%$ the universe age. Current environmental conditions are very different from the one present when the first proto living systems appeared or when the Last Universal Common Ancestor (LUCA) originated the current monophyletic lineage of life forms. Therefore, any theoretical development on living organization should address the basic organizational principles present in proto living systems [100, 101, 102. In other words, any valid theory of biological organization must explain, in the constrained chemical environment existing 4000 Mya, the appearance of rudimentary metabolisms that, after suffering a process of complexification, would generate the first living system and a persistent lineage lasting for almost 4 billion years. Thus our explanatory scheme begins forming a quasi-integrable Hamiltonian $\left(\mathcal{H}_{\epsilon\left(t_{0}\right)}^{\mathcal{B}}\right)$ from a background of basic primitive chemical reaction network to develop a pseudo or protometabolism. The kind of protometabolism at the beginning of the bio-genesis might be just a few chemical reactions; however, one of the main results of BOP is that they form a closed loop of reactions (with zero deficiency). This feature, associated with a quasi-integrable Hamiltonian becomes the signature of Biological Closure and a central topological motif in biological organization. This pseudo-metabolism can exist as a diffuse limited system and maintained by geophysical constraints as they are found in hydrothermal vents. Once a primitive metabolic network fabricates all its components it becomes an autopoietic system and a lineage of stable recurrent chemical interaction networks evolves associated with the environmental recurrences. One interesting question concerns how new small networks of chemical reactions are incorporated into the main autopoietic (closed) network without destroying systems' stability. Fortunately enough is known about extant metabolisms to develop the following picture. During reproduction by the processes of gene duplication or gene mutation, a new enzyme is generated and the ensuing offspring will have a metabolic network slightly different from its parents. Because of the Hamiltonian formalism and the application of KAM theorem, the resulting chemical reaction network will produce motions (i.e. temporal profiles of concentrations) 
for the different metabolites that are quasi-periodic and similar to the original trajectories thus endowing the original network with the properties of stability and robustness. In other words, the incorporation of some reactions will destroy the overall network while others will drift its overall activity, without destroying the underlying organization, depending on if they allow KAM Theorem to operate or not. This ideas, concerning the incorporation of a new character, seems copied from any text on evolutionary biology but our new insight is that the surviving networks are not selected but they, in fact, allow a variation and conservation on the organization as a mandatory consequence of KAM-theoretical dynamics. Thus the incorporation of a new network motif is not the selection (natural) of a random event but a deterministic predictable event derived from the nonlinear dynamics reflecting KAM-theorem. Thus form and variation result from the consequence of KAM-theorem and the integrability and quasi-integrability of the Hamiltonian $\left(\mathcal{H}_{\epsilon(t)}^{\mathcal{B}}\right)$ defining the duality system-niche and not from the hidden action of a selective process acting on phenomenological, a priori constructed, the fitness landscape of the system that ignores structural coupling. As a consequence, no selection, natural or any other, become necessary as evolution proceeds by a drifting expectation of the Hamiltonian of recursive system-niche interactions $\left(\mathcal{H}_{\epsilon(t)}^{\mathcal{B}}\right)$ while preserves its dynamical organization. Biological Closure and Structural Coupling constrains the dynamics to achieve KAM-surfaces as the system interacts with the medium while constructing its niche while continuously allowing small incremental physical interactions to be incorporated in the organized duality system-niche. Then, the evolutionary process appears as a natural drift [103] while following the KAM-theoretical dynamics with new quasi-periodic orbits split by Cantori sets.

Overall, the following important features concerning BOP and evolution/biogenesis must be underlined: firstly, the KAM-theoretical constrained dynamics maintains a conservative Hamiltonian organization defining a system-niche duality. Second, $\mathcal{H}_{\epsilon(t)}^{\mathcal{B}}$, which entails the duality system-niche, once established as an integrable Hamiltonian, its becomes preserved and initiates a monophyletic biogenesis process with conserved organizational features for the ensuing lineages. Third, cognitive (as anticipatory dynamics) and constructive (meaningful) system-niche duality follows from accommodating physical interactions in KAM-theoretical grounds. Fourth, the dynamical structure of KAM-organized $\left(\mathcal{H}_{\epsilon(t)}^{\mathcal{B}}\right)$, and then its system-niche duality, are endowed with local and global self-similar dynamics, fast, slow and complex time scales. Fifth, the dynamical trajectories of the KAM-theoretical dynamics are infinity as always new Cantori-bifurcations are theoretically accessible from already stable KAM-tori. These new accessible states are themselves robust and stable 
KAM-tori attractors capable of incorporating new small recursive interactions under Biological Closure and Structural Coupling. Finally, actively preserved integrability (or quasi-integrability) endows $\mathcal{H}_{\epsilon(t)}^{\mathcal{B}}$ to display the fundamental property of continuously reshaping symplectic manifolds, which in physical terms shows plasticity and adaptability, as a fundamental property of a living system in the evolutionary process of organizational and structural changes.

\subsubsection{Ontogeny and Phylogeny: Biological Structure of Space-Time}

Under DCM, the components and the networks forming a given living system, follow courses of transformations that preserve relational functional symmetries (system's organization) while allowing a continuous stream of interactions. Furthermore, the structure of internal and external perturbations impinging on living systems, by the phenomenon of Structural Coupling, becomes historically associated with the systems' organization forming ontogenetic and phylogenic lineages. Thus, for each organism, the particular history of interactions, either in an organism time span as well as in evolutionary time scales, is at the center of niche formation and niche inheritance.

Time. One of the most notable features of life is its relation to TIME. On one hand, living systems exist in their ontological time domain ranging from minutes (Bacteria) to centuries (Turtles) or even millenniums (Pinus longaeva/Patagonian cypress) but there also exist in evolutionary time scales, lasting about 3.5 billion years. Thus, any theory of biological organization must address the time domains of the individual as well as its evolutionary lineage. In other words, for living systems time represents some type of duality: as time implies, for the biological organization, discontinuity and also persistence. As we have mentioned, KAM-theoretical dynamics offers a multi-scale and complex dynamics associated with the unfolding of biological organization. Functional symmetries' interactions follow the trajectories in KAM-surfaces as such, microscopic, mesoscopic and macroscopic functional elements are allowed to follow fast, slow and mixed temporal dynamics. Moreover, all these interactions of functional symmetries convey diffusional processes that slightly move from chaotic trajectories toward well defined closed-loop deterministic interactions (integrable Hamiltonian). Temporal scales become dependent on the particular functional symmetries interactions implied, as well as the associated morphometric quantities; and the KAM-theoretical features which implement Arnold's diffusion web, the Chirokov or Nekhoroshev dynamical regimes either at local or global scales. Therefore TIME in biological systems is intimately associated with the organismniche duality as their interactions lie in different KAM-tori attractors dynamical regimes supported 
in which linear to super-exponential time scales are allowed. Time allows the biological organization to unfold into the physical world as an organismal unit where time-frames are overlayed not as a physical dimension but as a mechanism resulting from linking processes at different dynamical scales. Without this time-mechanism, biological systems will trend to simple dissipating diffusional processes. Notably, living systems organization does not occur in the full phase space in an ergodic manner. In effect, the organization trajectories lying in KAM-surfaces, where the quasi periodic trajectories occur, become intermixed with chaotic regimes where organization becomes lost. Additionally and more importantly, Biological Closure and Structural Coupling impose constraints in the morphometric quantities linking organization and physical interactions in the structural states of functional symmetries. Therefore, the interaction trajectories between functional symmetries are also constrained in phase space by Biological Closure and Structural Couplingand not allowed full phase space exploration.

\subsubsection{Variational Structure and Function}

Structure, Shape and Structure-Function relationship. Living systems display a wide diversity and conservation of anatomical structures. These anatomical features are robust, prominent, and -since Linnaeus- are the basis for taxonomical classification. Additionally, variation, convergence, and adaptability dominate the repertoire of functional features related to structural features. Usually, a function is used to associate specific relations of usability to the structure; whereas structure can be either defined in the physical context of material interactions as well as separated elements associated to the organizational context where a more abstract entailment is needed. Nevertheless, structure and function, in the context of BOP emerge from the association of organization of living systems with their system-niche duality. Thus a theory of living organization should account for the conservation and diversity of living systems' structure [104].

Organization and Structure. A fundamental distinction in early autopoietic theory is the distinction between Organization and Structure. This distinction is important as Maturana and Varela used both terms quite atypically. In effect, according to Maturana and Varela [1,3] Organization, is the set of processes that make an organism a member of a class, while Structure is the set of processes and components that constitutes the physical concrete organism: Organization is a subset of Structure. We think that this powerful distinction must be reinterpreted. In our view, living systems are physical systems with the essential feature of system-niche duality and where Organi- 
zation could be interpreted as a mathematical abstraction with differential geometry components. In effect, in BOP framework living systems are understood primarily as dynamical systems in a differential geometry context and where all the features of the organization are related to abstract Geometric Organizational Structures. Poisson brackets, Symplectic structure, and Stochastic and Hamilton-Lagrange Mechanics are the structural elements of Organization. Thus, under the viewpoint of BOP, Biological Organization is understood as complex mathematical construction with many common elements used to understand modern physics and mathematics.

On the other hand, Structure, in BOP framework, must be understood in a more general sense as the relational composition of the elements either in physical or organizational space. In other words, structural elements are components featuring properties associated with the kind of elements they identify: relational and mathematical abstractions for organization and physical features for material entities. Furthermore BOP incorporates two elements not found in early autopoietic writings: Functional Symmetries and Morphometric features. Where these new elements belong? Functional symmetries and morphometric features emerge in the context of analyzing the structure and function relationships that appear at many levels in biology. From molecular elements as proteins, entailing catalytic activity, to predator-prey interactions in ecological systems and excitationinhibition balance on the excitability of nervous systems. In effect, what become clear is that all the above elementary interactions emphasize the binary nature of functional observations on living systems (excitation/inhibition, prey/predator, substrate/product, open/close, active/inactive, etc..). A more in-depth analysis shows that all these binary relationships are a simplified form of functional states of physical structures (genes, proteins, populations, etc..). Moreover, always these physical structures can be described by arbitrary probability density functions along a functional axis. BOP incorporates this analysis in the notion of functional symmetries as the many - but constrained- configuration states of the physical structure across organized levels as well as its implicit functional entailment. Morphometric features account for the local unfolding of functional symmetries in each organizational domain or descriptive level and thus represent the quantitative measure of the probability density function associated with functional symmetries.

\subsubsection{Functional Symmetries}

We have abstracted biological components/elements into functional symmetries. The usual focus in biological research is on physical aspects of an organism, as for example the shape, molec- 
ular components and relations between them in all scales. The systematic use of this approach has yielded the mechanic-reductionist viewpoint dominating current biological research. Using an abstract systems approach to define organization we introduced functional symmetries. These functional symmetries objects are not new to biology; they usually represent a generalization of the properties of a set of objects of similar properties. In the molecular scale, proteins are naturally grouped according to their functional features: enzymes, structural scaffolding, molecular recognition, templates, etc. The functional symmetries abstraction targets on one side the evident functional role of this kind of objects where the functional states, in most cases, is a binary active or inactive state of a biological function. Whereas on the other hand, the symmetries came as twofold; the functional state is achieved as a defined state of the physical structure of the object attained in the active functional state. For example, phosphorylation of regulatory sites switch/activate an enzymatic activity; the binding of a specific co-factor triggers a cascade of physical interaction of "signaling". In all these cases, a dynamical structure with the functional role is assembled by a particular configuration or disposition of material elements/parts. The other, the symmetry property, is attained in the organizational realm and it is related to the formal definition of the material object and its relation to the organization. The organizational realm is conceived as the relational properties of the material objects, transcending the particular embodiment in the physical realm. In this case the symmetry is related to the transformations that maintain an invariant at the organizational level. The invariant property establishes relations (of order) between the components for a given organization. The invariant property is the biological function served by the physical structure. Notably, the functional symmetries act as layers of abstraction to different hierarchical levels of biological organization. We use the transformation scheme in equation (7) to represent the organizational invariant of the functional biological role. The functional symmetries, derived from (7), are represented by a position and its time derivative, a Lagrangian system in a mechanical particle and inertia. The inclusion of forces, in Biological Organization the systemic interactions due to Biological Closure and Structural Coupling, leads to a Hamiltonian system with position $(q)$ and momentum $(p)$. The duple $(p, q)$, entailed by functional symmetries, bridges the distinction between Organization and Structure and reflects the micro-dynamics of variables/parameters that are essential for Hamiltonian systems.

For BOP the distinction between organization and structure is not as crucial as in early Autopoietic theory which was heavily influenced by 1960s Cybernetics. On the contrary, BOP assumes 
that physical objects (i.e. components in the parlance of Autopoiesis and thus pertaining to the structure) are the end result of a dynamical process. BOP explains structure-function relationship by considering an integrated interaction between the organization and physical features found in living systems. As the duality system-niche is the central evolving and defining element, DCM incorporates into the system physical structure the recursive system-niche interactions in the formation of $\mathcal{H}_{\epsilon(t)}^{\mathcal{B}}$, the preserved organization. Then structure-function relationships are the final result of the recursive history of system-niche interactions incorporated in the identity of the system by allowing small incremental variations on its organization as a defined class of systems, as observed by Maturana and Varela [1,3]. This structure-accommodating feature appears as an adaptive element in the structure-function of living systems, which has been interpreted as a selection over variation. However, in the BOP framework the structure-function is a reflection of the system-niche duality and a central constraint in the maintenance of system organization. Ultimately, the integrability and quasi-integrability allow the system to accommodate the variations of the morphometric features implementing the conservative dynamics of the organization.

\subsubsection{Cognition as construction}

Behaviour. Living systems are dynamical systems that act upon the medium, since von-Uexkull 105] we know that this acting is not a mere manipulation of an objective reality but rather the specification of the organism's living space (the Umwelt is produced by the history of organismmedium interactions. Understanding how this specification occurs is a central aspect of any theory of the living system organization. How the surrounding objects, including the relations among them, are specified by the internal dynamics of living systems. BOP illuminates this problem by clearly stating that the organizational trajectory of a living system is the result of the recursive interaction between it and its environment and thus objects are behaviorally defined, in actionperception loops, and constitute the duality system-niche. This occurs as the interactions become constrained to either forming the Hamiltonian that represents its particular organization, $\mathcal{H}_{\epsilon(t)}^{\mathcal{B}}$, as well as the trajectories of interaction to lie in the KAM-surfaces. Then, action-perception loops become incorporated in a single trajectory of functional symmetries coupled in a KAM-theoretical surface which it's been enclosed by organizational plasticity. 


\subsubsection{BOP's Mathematical-Physics Novelties}

We have introduced a well known theoretical framework with methods used for mechanical systems to understand the biological organization. We have found, in general, that many theoretical and methodological developments in Differential Geometry are suitable to understand living systems' organization. We underlie the use of Hamiltonian formalism and the KAM-theorem as cornerstones to understand biological phenomena in a new theoretical context. We have done more than a simple translation of these ideas to biology as we have introduced what we think is a genuine new set of features to understand the dynamics of complex systems. Besides the use of Differential Geometry frameworks, the idea of a plastic Hamiltonian continuously shaped by stochastic path integrals in the form of an operator is a new mathematical development for the theory of Hamiltonian dynamics. Likewise, the representation of this time-dependent Hamiltonian operator as an asymptotic stochastic integral seems to be closely related to quantum's wave functions and thus opening new interesting routes of analysis. Symplectic structures also may offer new light to understand complex Hamiltonian embeddings which coherently appear supporting KAM-theoretical dynamics. Most notably, KAM-theorem appears as underpinning many complex biological phenomena, it conveys not only long term stability but also implies the incorporation of recurrent perturbations into dynamical structures. In Biological systems, KAM-theorem also is implied in reproduction, lineage formation and in distributing energy-matter-information in non-linear biological systems interactions. The ideas of manifolds, Markovian processes, and stochastic phenomena illuminate the microscopic level where DCM is partially at play. Interestingly, the mechanical actions underlying deterministic trajectories can be decomposed into local stochastic processes. These processes form a multi-dimensional interaction with the medium where the action-perception loop becomes a cognitive sensory-motor coordination. The cognitive action-perception is then ruled by the history of system-medium interactions, which are incorporated into the organization defining the system as a lineage. Our framework proposal also introduces stochastic path integrals as they were developed originally by Feynman for quantum mechanical systems. The use of stochastic path integrals in our framework, however, is novel as it represents the stochastic dynamics of structural coupling. The microscopic and intrinsically random nature of fluctuations at molecular scales, as well as the unknown factors influencing the states of highly complex processes (metabolic interactions for example), make a particularly suitable use of stochastic path integrals to define the sequences of interactions and transformations of functional symmetries. Moreover, these developments in our 
framework lead naturally to the inclusion of biological closure to constrain even more the organizational trajectories followed by functional symmetries. Then, Biological Closure and Structural Coupling, becomes associated with a mechanistic core (DCM) to establish the organization and structure of living systems. Overall the central core, Dynamical Closure Mechanism (DCM), orchestrates several mathematical-physics concepts and methods which together constitute a new formal point of view to understand the biological organization. DCM is a single unitary mechanism underpinning the multiple and parallel processes at play across hierarchical levels of complexity to produce a biological organization.

\subsubsection{Biological Organization as Mechanical Trajectories}

The use of mathematical-physic tools and concepts not only grounds biological organization as an idealized mechanical system, while also conveying the dynamics of the systems into the mechanical evolution laws. Thus, the dynamics of trajectories associate two complementary points of view; the stochastic paths integrals describing trajectories of organizational interactions mediated by random interactions and the deterministic trajectories of phase-space for the macroscopic interactions. Intrinsic to these complementary descriptions is a fundamental concept to our framework: the macroscopic stability of the organization and the inherent variability associated with a spontaneous change in the organization coupled to the medium in relation to natural drift. The stochastic variability represented by a statistical ensemble of path integrals subject to the recurrence of perturbations, intrinsic and extrinsic, leads to a drifting mechanism that slightly and continuously modifies the organizational dynamics.

\subsubsection{Complexification of the Biological Organization by a Simple Common Core, DCM}

We have introduced a basic mechanistic core (DCM) that, starting from interactions between functional symmetries -which continuously modified themselves- as well as organism-medium interactions forms a relational organization, the physical structure and specifies the system's dynamics. The continuous operation of DCM and the parallel interactions of functional symmetries make the organization to exist and assemble Hamiltonian embeddings of symplectic manifolds. These embeddings form complex structures in the organizational realm; however, they are not directly accessible by analytical methods. Instead, they can be addressed by modern computational methods [58] which are not in use currently in biology. Nonetheless, other interesting approaches can be used in more simple geometrical settings: for example, port-Hamiltonian analysis is amenable 
to reduced metabolic networks and hybrid Hamiltonian systems in more familiar development to systems biology.

\subsubsection{Inclusion of Other Theoretical Frameworks}

The Hamilton-Lagrange mechanic and dynamical framework of mathematical-physics are considered elegant, powerful and general. Informational, thermodynamical and statistical frameworks can also be tied to the Hamiltonian formalism as they emphasized aspects of this mathematical construction. The Kullback-Leibler divergence, entropy, thermostats assemble, phase transitions, expectations, probabilities, Bayesian inference and so forth are part of a toolbox of methods and complementary descriptions that can be used to emphasize certain theoretical relations in modern dynamics [21]. One of the most developed theories using some portion of these concepts is the Free Energy Principle (FEP) by Karl Friston and collaborators which exploits the notions of variational minimization functional, Bayesian networks and inference, information entropy and thermodynamics energy metaphors to propose a theory of living systems and brain function. As such, FEP is broad in scope as well as from its theoretical point of view and adheres to autopoiesis as a central feature of living systems. Nevertheless, FEP does not address a mechanism, although it is conceived as a dynamical system featuring and relaying in axiomatic principles using a thermodynamic-informational metaphor. Our framework broadens the theoretical developments of FEP, not only in the mathematical-physical sense but also in its biological extensions to origin (biogenesis), evolution, diversification, and conservation of living systems.

\subsubsection{Reevaluation of Autopoiesis and Cognition Theory and perspectives}

BOP framework implements a quantitative formulation for autopoiesis and its underlying cognition theory based on structural coupling. The qualitative developments of Autopoiesis and Cognition Theory have been numerous and broad in scope although the quantitative approaches have been limited [34, 106]. Our framework re-visits all these quantitative attempts and many of the qualitative developments with a new perspective. BOP transforms the qualitative definition of an autopoietic system into a more precise formulation involving tools from mathematical physics. Our formulation is a definitive improvement as autopoietic theory, since its inception almost 50 years ago, has been limited to very general statements. Besides the metaphorical use of autopoiesis in fields ranging from Architecture to Sociology all the attempts to expand autopoiesis into a workable theory of living systems (like Varela's use of Indicational Calculus or the use of Rosenean notions 
have only produced limited advances. Importantly, our proposal contains a core mechanism for Biological Closure and Structural Coupling, the Dynamical Closure Mechanism (DCM), the applicability of DCM to the current qualitative developments of autopoietic and cognition theory can contribute to advance those extensions as the mechanism is general enough to fit a broad range of scenarios and tackle them with numerical methods.

One interesting application of our framework is in the field of neurosciences as nervous system excitability becomes one of the most convenient and interesting test platforms for our proposal. Neuroscience has greatly progressed in the quality, sophistication and technical possibilities enabling then the testing of some aspects of our framework. BOP can be studied in a wide spectrum of situations from the biophysical mechanisms of neural function and regulation associated to the functional symmetries (see for example [29], to neurodynamics and inferential Bayes as it is been studied by Friston and colleagues). For example, the general Bayesian brain approach, which is essentially in agreement with our point of view, added to the current ability to record global patterns of neural activity, for example via EEG, provides theoretical constraints to our model on cognition, neurodynamics and signal structure in the nervous system. These tests would involve the embedding of EEG signals in high-dimensional spaces (as it has been done by Freeman) but instead of looking for signal descriptors (like the Lyapunov exponent) the quasi-periodicity of EEG would be interpreted as the construction of a Hamiltonian by diffusion. The combination of behavioral learning tasks with recordings of neural activity in the context of quasi-integrable Hamiltonian flux dynamics. In this context, behavioral learning must be accompanied by decreasing chaotic activity and increments on the trajectories of neural activity quasi-periodic as well as lower Lyapunov estimates. Similarly, the comparison between active and inactive behavioral states would allow observing key features of Hamiltonian dynamics. Another way to further test Biological Organization Principles framework is in computational modeling and simulation of neuronal networks and its dynamical changes associated with learning and memory in the context of AI.

Another area where a closer examination can be useful to test ideas is on the origin and evolution of living systems. In effect, our framework provides novel scenarios to test pseudo-metabolisms of an ancestral type. New technologies in synthetic biology may render additional clues on the formation and evolution of simple metabolic networks and molecular cognitive devices. In effect, our emphasis on functional symmetries and morphometric quantities constrains boundaries and direction of change for metabolisms acquiring (or losing) reactions. The net effect of this transformation 
is to change the overall network and thus to change the underlying Hamiltonian. We claim that a metabolic theory (not based on the current ideas of Steady-State solution) could be built around this idea of a plastic Hamiltonian. Furthermore, this change must be coupled to the structure of the environmental change. According to BOP the linear availability of substrate molecules would lead to a continuous adjustment on steady-state dynamics whereas that oscillations and periodicity in the metabolism would be prone to be incorporated in structural changes as catabolic/anabolic metabolic pathways formation. Biological models would include from extremophiles to specialized organisms. It should be noted that metabolic Hamiltonian definition in eukaryotes includes proteins, genetic and epigenetic factors in a complex web dynamics.

In general, our framework provides the grounds to establish the interaction networks of functional symmetries, which by incorporating small perturbations become closed, forming a quasiIntegrable Hamiltonians. Rate equation and master equations of interaction networks at a small number of interacting component, as its for small metabolic pathways as well as compartmentalized biophysics, yield low dimension matrix associated with the Hamiltonian formulation. Larger networks, as the full cellular metabolism or neuronal circuits, require numerical approaches to guide experimental parametrizations and complex modeling of interactions. However, the most significant results of big-modeling strategies can be seen as in the development of deep neuronal networks where functional complex behaviors are modeled. In the metabolic counterpart, biomedical and therapeutic modeling may help to design novel drugs or procedures for complex molecular conditions. Thus Biological Organization Principles framework provides keys to understand the complexity of biological networks of many types and organizational levels either theoretically or experimentally.

\subsection{Final Summary}

We have presented here a wide-ranging framework to understand the dynamics and evolution of biological systems anchored on powerful ideas from biology and mathematics. Our approach could appear as over-reaching for such an open and complex topic, but we think that we have found a particularly potent approach based on a) the notions of Biological Closure and Structural Coupling and b) the use of abstract ideas of differential geometry, particularly non-linear Kolmogorov equations, stochastic mechanics and KAM theory. Unexpectedly the use of mathematical tools, which are commonplace in Physics, in conjunction with BC\&SC permits to understand a new essential mechanism (DCM) that serves to control the micro and macro dynamics of living systems. 
It could be argued that our scope is too general, without clear cut technical results and perhaps excessively complicated as many novel tools and ideas are used in parallel. We think that this spectrum of ideas and tools is necessary and it reflects a necessary new path in advancing theoretical biology. The principal advantages and pitfalls of our analysis are thus underlined:

Living systems as multidimensional dynamical systems. Without any doubt the theory of nonlinear dynamics represents a great leap forward to understand systems endowed with dynamics represented by differential equations. Also, without any doubt, the summit of this intellectual effort is the famous KAM-theorem that establishes the condition of stability and conservation for perturbed Hamiltonian systems. This rather surprising theorem states that for some class of dynamical systems suitable perturbations transform a stable regime of motion into another stable regime. We assert that the KAM theorem could be used to understand living systems in spite of their bewildering and multifaceted complexity. This is an important result that, at least, serves to illuminate the dynamics of living systems from a new qualitative viewpoint. Nevertheless, higher order differential geometry and numerical methods also may yield significant results as the high dimensionality of biological interacting networks makes impossible to approach this problem analytically beyond KAM-theory. Besides KAM-theorem, a crucial aspect of our approach is the incorporation of the biological ideas of Biological Closure and Structural Couplinginto a formal approach. BC\&SC maps very interesting aspects of the mathematical process, which are usually obscured in the common treatment of complex dynamics. Although quasi-integrability in Hamiltonian systems is very well studied, it does not incorporate a mechanism of asymptotic formation, nevertheless biological phenomena makes extensive use of this notion in the variability and biological evolutionary features. On the other hand, Structural Coupling makes it possible to incorporate the dynamics of perturbations in defining the mathematical structure of the underlying system, in a way that continuously defines boundary structures and dynamics (as a cognitive process). Overall, Biological Closure and Structural Coupling can be translated into powerful mathematical structures which, in combination with complex physical phenomena (turbulence), form a new type of systems where energy and matter can be organized. Moreover, Biological Closure and Structural Couplingmay open the path to a new relationship between statistical mechanics and chaotic and deterministic systems. 
Understanding vs manipulation. The BOP framework puts in the same footing the understanding of Physics and Biology as it uses a common set of concepts and tools to provide a coherent understanding of Biology and illuminates the physics of complex systems. These are an important achievement for our framework, as no new abstract entities ad-hoc to biology are needed. Moreover, Differential Geometry in BOPuses a framework already consistent from quantum mechanics to cosmology with paramount extensions to technology and applied sciences; the concepts used in BOP framework then have been shown to be useful already in simple theoretical and experimental cases. In physics, where systems are defined by a few classes of objects and properties, Hamiltonians are simple functions that are usually guessed or derived for simplified systems. Our approach is vastly different and more perceptive as the Hamiltonian for the living system is not a simple expression involving systems variables (Energy, Entropy, Temperature, quantities of components) but the end result of a constructive process (DCM) making the Hamiltonian of living systems a limit rather than an a-priory expression. The process by which the limit is computed, and thus how $\mathcal{H}_{\left\langle\epsilon\left(t_{0}\right)\right\rangle}^{\mathcal{B}}$ is calculated, represents an important advance as it uses in a new context sample path integral methods and probability theory. Three main novelties can be underlined; in physics, we introduced Hamiltonians build by a limiting/asymptotic processes; and in biology, we use Hamiltonians to represent the organization as well as construct them by using sample path integrals and Markovian processes in stochastic paths. Another important advance refers to the use of functional symmetries as the relevant parameters that any theory should consider. We think that from the plethora of internal variables found inside living systems the variables defined by partition equilibrium $((1-X) \leftrightarrow X)$ are the relevant variables that should be incorporated into any theory about the organization. These simple binary equilibrium can be generalized to functional symmetries having multiple equilibria (multi-modal or arbitrary partitions). Another point is, although our framework does not identify the relevant functional symmetries forming the $\mathcal{H}_{\left\langle\epsilon\left(t_{0}\right)\right\rangle}^{\mathcal{B}}$ the inclusion of any possible configuration of interacting symmetries can be bounded by the zero deficiency of the network, implied by the constraints derived from BC\&SC. Additionally, although the temporal scales are not given in advance the non-linear Kolmogorov equations force to consider a perturbation wave of activity to propagate in a heterogeneous media and which defines the equilibrium time constants for the system. Importantly, $\mathcal{H}_{\left\langle\epsilon\left(t_{0}\right)\right\rangle}^{\mathcal{B}}$ is endowed with plasticity and, crucially, the stochasticity associated to BOP becomes stabilized by the interactions with the medium via DCM and KAM-theorem while remaining a deterministic operator. 


\subsection{1. homine digitus}

Our BOP theoretical approach is centered around a fundamental idea: Living systems can be described as mechanical systems with an extension of the fundamental concepts in mathematicalphysics and differential geometry and by considering Biological Closure and Structural Coupling. The Hamiltonian defining biological organization $\left(\mathcal{H}_{\epsilon(t)}^{\mathcal{B}}\right)$ is dynamically constructed by the unique path of encounters of every organism and its medium. Then, mechanic's tools are useful to describe this historical interactive construction using, like building blocks, functional symmetries, probability distributions, path integrals and so forth.

The following scheme serves as a guide to our overall BOP framework, first a stochastic Hamiltonian $H_{s t}^{\dagger \circledast}$ is defined by:

$$
H_{s t}^{\dagger \circledast}=\int_{E_{0}}^{E_{t}} e^{-S^{\dagger \circledast}}\left\langle\left\langle P^{\dagger \circledast}\right\rangle\right\rangle_{x(t)}
$$

Here a modified path integral weight the action $\left(S^{\dagger \circledast}\right)$ of each functional symmetry $(x(t))$ in the trajectory route $\left(P^{\dagger \circledast}\right)$ and forms a stochastic Hamiltonian. The modified sample path integral, is convolved in action and trajectory by the continuous history of interactions with the medium, which in all instances is represented by the ${ }^{\circledast}$ operator. Thus far, no departures from regular physical systems occur and the results are chains of Markov process supported by the functional symmetries been perturbed mechanically by environmental forces. Then biology can begin to appear, as Biological Closure and Structural Coupling simultaneously operate in this stochastic background of interactions. At first, for illustrative purposes, we can focus on structural coupling. Some interesting ensemble of functional symmetries begin to co-variate with external perturbations $(\epsilon(t))$, initiating a forward Kolmogorov chain of interactions which also by the structure of the recurrent interactions and the functional symmetries propagate (loop) backwardly but without reaching the initial condition. Then Biological Closure and Structural Coupling begins to operate making more refined forward and backward activity propagations. This allows, in the space of stochastic interactions in equation (24), an ensemble of nearly closed trajectories coupled to $\epsilon(t)$ to appear. Biological Closure and Structural Coupling further refine this condition as expectation over some limits. Specifically, minimizing the difference in structural coupling, i.e. as $\epsilon(t)$ goes to zero and biological closure takes quasi-closed trajectories of interaction for the functional symmetries at longer times, condition represented as

$$
\mathcal{H}_{s t}^{\mathcal{B}}=\lim _{\epsilon t, t \rightarrow 0, \infty} \epsilon(t)
$$


Equation 25) already is incorporating Biological Closure and Structural Coupling and also yields the Biological Hamiltonian from a stochastic setting $\left(\mathcal{H}_{s t}^{\mathcal{B}}\right)$. Combining equations (24) and (25) we obtain the following expression:

$$
\mathcal{H}_{s t}^{\mathcal{B}}=\lim _{\epsilon, t \rightarrow 0, \infty}^{\left\langle H_{s t}^{\dagger \circledast}\right.} \epsilon(t)=\oint_{E_{0}}^{E_{t}} e^{-S^{\dagger \circledast}}\left\langle\left\langle P^{\dagger \circledast}\right\rangle\right\rangle_{x(t)}
$$

This action integral is special, and this explains the special symbol denoting it, as it is taken around quasi-closed trajectories and therefore over asymptotically closing spirals. The time evaluation, as it tends to infinity, allows the Kolmogorov (forward and backward) equations to loop closer and closer from some arbitrary starting point, reflecting Biological Closure. Then an activity chain produces a quasi-closed sequence of activation and a wave of activity is produced by the small perturbations among functional symmetries.

Another interpretation for the formation of $\mathcal{H}_{s t}^{\mathcal{B}}$ is as follows: whereas Biological Closure and Structural Coupling constrains the ensemble to a particular stochastic Hamiltonian $\left(\mathcal{H}_{s t}^{\mathcal{B}}\right)$ taking into account the expectation-asymptotic paths integral evaluation; $\mathcal{H}_{s t}^{\mathcal{B}}$ also needs to reach Biological Closure in the form of integrability and simultaneously achieve Structural Coupling with the medium. These conditions are cast in the formation of the Biological Organized Hamiltonian in the relation

$$
\mathcal{H}_{s t}^{\mathcal{B}}=\lim _{t \rightarrow \infty}\left\langle H_{s t}^{\dagger}(\epsilon(t))\right\rangle+\epsilon(t) \circledast \mathbf{H}(\mathbf{q}, \mathbf{p})
$$

Here, as the asymptotic limit is formed the perturbation becomes evaluated by the convolution of the physical Hamiltonian $(\mathrm{H}(\mathrm{q}, \mathrm{p}))$ in the surroundings of the system. The spectral content of perturbation $(\epsilon(t))$ convolves the physical environment entailed by $\mathbf{H}(\mathbf{q}, \mathbf{p})$, extracting the recursive interaction system-environment and forming its niche as it becomes incorporated in $\mathcal{H}_{s t}^{\mathcal{B}}$ : then transforming interactions to an organization. A simple more compact nomenclature is:

$$
\mathcal{H}_{d t}^{\mathcal{B}}=\mathcal{H}_{\left\langle\epsilon\left(t_{0}\right)\right\rangle}^{\mathcal{B}}+\epsilon(t) H(I, \theta)
$$

The crucial point here is that, now $H(I, \theta)$ (a canonical transformation of $\mathrm{H}(\mathrm{q}, \mathrm{p})$ ) has been selected in the initial structural content of perturbations $\left(\epsilon\left(t_{0}\right)\right)$ reaching the functional symmetries in structural coupling and then $\epsilon \approx 0$, from this reasoning it should be clear that, following KAM-theorem, equation 28] holds. 
Finally, the complex structure formed by the recursive system-environment interactions is a complex embedding space. The embedding reflects the phase space of KAM-theoretical dynamics as well as the topological roughness of the overall stochastic interactions. The interactions, internal and external, and the embedding process construct the specially distinctive feature, departing from the Hamiltonian normally used in Physics, of continuous plastic accommodation of the embedded Hamiltonian. The plastic transformation of Hamiltonian as well as the system-environment interactions make the overall system a cognitive process accommodating environmental recurrences and the self-modification of the Hamiltonian in a continuous drift as indicated by the plasticity equation

$$
\frac{\partial}{\partial t} \Psi\left(\epsilon(t), \mathcal{H}_{\epsilon\left(t_{0}\right)}^{\mathcal{B}}, t\right)=\mathcal{H}_{\epsilon(t)}^{\mathcal{B}}(\Psi(t))
$$

In this case, $\mathcal{H}_{\epsilon\left(t_{0}\right)}^{\mathcal{B}}$ represents a lineage of recursive interactions system-environment and the systemniche duality. The operator $\Psi$ represents another view of Biological Closure and Structural Coupling as it takes $\mathcal{H}_{\epsilon\left(t_{0}\right)}^{\mathcal{B}}$ at a given time and, by evaluating the perturbations $\epsilon(t)$, updates $\mathcal{H}_{\epsilon(t)}^{\mathcal{B}}$ which acts upon the operator $\Psi$. This viewpoint shows that $\Psi$, which acts upon $\mathcal{H}^{\mathcal{B}}$ and is acted by $\mathcal{H}^{\mathcal{B}}$, represents the system's structural plasticity.

The complex navigation on these topological dynamics is shown in the equation (solution of 29, this solution involves the non-ergodicity of the system, the ontological construction of the organization and structure, the initial conditions and the environmental interactions. Then the final result is a highly complex ensemble of multidimensional interactions that forms the organism, its niche, its cognitive dynamics and its understanding of the physical structure in the environment adjacent to its immediate actions.

$$
\Psi\left(\epsilon(t), \mathcal{H}_{\epsilon\left(t_{0}\right)}^{\mathcal{B}}, t\right)=\exp \left(t \mathcal{H}_{\epsilon(t)}^{\mathcal{B}}\right)\left(\Psi\left(t_{0}\right)\right)
$$

The trajectories deduced from equation (30) undergo many dynamics (mainly KAM) weighed exponentially toward its stable regime formed by the plasticity process (equation (29)). In other words, the actual perturbations $(\epsilon(t))$ are continuously compared to the historical perturbations present in the structure of the organism lineage, $\left(\mathcal{H}_{\epsilon\left(t_{0}\right)}^{\mathcal{B}}\right)$ and exponentially incorporated in the organization as a small variation as they become validated by operator at the lineage $(\Psi)$ defining the evolution of the lineage.

This Biological Organization Principles framework summary should be taken as the architectural logic for a full mathematical demonstration of how KAM-theoretical dynamics explain Biological 
Organization, Evolution, Plasticity, and Cognition. Although demonstrations could be brief or lengthy, the logical structure will be as it has been posited here. The preliminary assessment introduced here needs some extension in how some physical concepts, especially the Hamiltonian energy dogma, are used. Also, the mathematical community must be invited to consider Biology not only as a place to use mathematics but also a place where mathematical ideas can be born especially at the very frontier of non-linear dynamics, differential geometry and related fields as a consequence of the biological notions of Biological Closure and Structural Coupling. In fact, selfreference, a property studied in many mathematical sub-fields, is one of the leitmotiv of Biology. Thus, creating a deep and mostly hidden connection between Mathematics and Biology. Finally, the equations presented here engage much more than technical results or a particular theoretical framework, as they embody a wide research program integrating Physics, Mathematics, and Biology aiming far beyond current attempts to understand Biological Organization. 


\section{References}

[1] H. Maturana, F. Varela, De maquinas y seres vivos, Editorial Universitaria, Santiago, 1972 (1972).

[2] H. Maturana, The organization of the living: A theory of the living organization, International Journal of Man-Machine Studies 7 (3) (1975) 313-332 (1975). doi:http://dx.doi.org/10. 1016/S0020-7373(75)80015-0.

URL http://www.sciencedirect.com/science/article/pii/S0020737375800150

[3] H. R. Maturana, F. J. Varela, Autopoiesis and Cognition : the realization of the living, D. Reidel Publishing Company, 1980 (1980).

[4] K. J. Friston, T. Shiner, T. FitzGerald, J. M. Galea, R. Adams, H. Brown, R. J. Dolan, R. Moran, K. E. Stephan, S. Bestmann, Dopamine, affordance and active inference, PLoS Computational Biology 8 (1) (2012). doi:10.1371/journal.pcbi.1002327.

[5] K. Friston, Life as we know it. Journal of the Royal Society, Interface 10 (86) (2013) 20130475 (jul 2013). doi:10.1098/rsif.2013.0475.

URL http://rsif.royalsocietypublishing.org/cgi/doi/10.1098/rsif.2013. 0475http://www.ncbi.nlm.nih.gov/pubmed/23825119http://www.pubmedcentral . nih.gov/articlerender.fcgi?artid=PMC3730701

[6] B. Calcott, K. Sterelny, The Major Transition, MIT Press, 2011 (2011).

[7] B. K. Hall, R. D. Pearson, G. Muller, Environment, development, and evolution : toward a synthesis, MIT Press, 2004 (2004).

[8] J. H. Woodger, Biological Principles: A Critical Study, International library of psychology, Harcourt, 1929 (1929).

URL https : //books.google.cl/books?id=SkkVAAAAIAAJ

[9] T. A. C. Reydon, L. Hemerik, Current themes in Theoretical Biology: A Dutch Perspective, 2005 (2005). doi:10.1002/9781118568170.ch49.

[10] A. Barberousse, M. Morange, T. Pradeu, Mapping the Future of Biology: Evolving Concepts and Theories, Vol. 266, 2009 (2009). doi:10.1007/978-1-4020-9636-5.

URL http://dx .doi .org/10.1007/978-1-4020-9636-5 
[11] J. S. Torday, W. B. Miller, The Resolution of Ambiguity as the Basis for Life: A Cellular Bridge between Western Reductionism and Eastern Holism, Progress in Biophysics and Molecular Biology (2017) 1-34 (2017). doi:10.1016/j.pbiomolbio.2017.07.013.

URL http://dx.doi.org/10.1016/j.pbiomolbio.2017.07.013

[12] A. O'Hear, Philosophy, Biology and Life, Oxford University Press, 2005 (2005). doi:10. 1017/CB09780511599729.

URL http://ebooks . cambridge.org/ref/id/CB09780511599729

[13] P. Huneman, Understanding Purpose: Kant and the Philosophy of Biology, Vol. 8, University of Rochester Press, 2007 (2007).

[14] H.-k. Chao, S.-t. Chen, R. L. Millstein, Mechanism and Causality in Biology and Economics, Vol. 3, 2013 (2013). doi:10.1007/978-94-007-2454-9.

URL http://link. springer.com/10.1007/978-94-007-2454-9

[15] A. Gambarotto, Vital Forces, Teleology and Organization. Philosophy of Nature and the Rise of Biology in Germany, Vol. 21, 2018 (2018). doi:10.1007/978-3-319-65415-7.

URL http://link.springer.com/10.1007/978-3-319-65415-7

[16] M. Grene, D. Depew, The Philosophy of Biology: An Episodic History, The Evolution of Modern Philosophy, Cambridge University Press, 2004 (2004).

URL https://books . google.cl/books?id=X83LCgAAQBAJ

[17] P. Schuster, Is There a Newton of the Blade of Grass?, Complexity 16 (6) (2011) 3 (2011). arXiv:arXiv:1103.4838v1, doi:10.1002/cplx.

[18] R. Winfield, Universal Biology after Aristotle, Kant and Hegel: The Philosopher‘s Guide to Life in the Universe, 2018 (2018).

[19] W. Greiner, Classical Mechanics: System of Particles and Hamiltonian Dynamics, SpringerVerlag Berlin Heidelberg, 2010 (2010). doi:10.1002/1521-3773(20010316)40:6<9823:: AID-ANIE9823>3.3.C0;2-C.

URL papers3://publication/uuid/307983B2-11E0-4D20-AC36-85CE20264D19

[20] K. Simonyi, A Cultural History of Physics, no. 5, CRC Press, 2012 (2012).

URL papers3://publication/uuid/49939CFD-D871-4AB4-B224-4F4EBC47913E 
[21] D. D. Nolte, Introduction to modern dynamics : chaos, networks, space and time, 2015 (2015). URL https://global .oup.com/academic/product/introduction-to-modern-dynamics-9780199657032? lang $=$ en $\{\&\} c c=$ in

[22] A. N. Kolmogorov, On the conservation of quasi-periodic motions for a small change in the Hamiltonian function, Doklady Akademii Nauk 98 (4) (1954) 527-530 (1954).

URL http://cds.cern.ch/record/430016

[23] V. I. Arnol'd, Proof of a theorem of AN Kolmogorov on the invariance of quasi-periodic motions under small perturbations of the Hamiltonian, Russian Mathematical Surveys 18 (5) (1963) 9-36 (1963).

[24] J. Möser, On invariant curves of area-preserving mappings of an annulus, Nachr. Akad. Wiss. Göttingen, II (1962) 1-20 (1962).

URL http://cds.cern.ch/record/430015

[25] R. Rosen, Life itself : a comprehensive inquiry into the nature, origin, and fabrication of life, Columbia University Press, 1991 (1991).

[26] A. Bodganov, TEKTOLOGY: The Universal Organizational Science, 1996th Edition, no. March, Centre for System Study Press, Hull, UK, 1913 (1913).

[27] J. V. Uexküll, A stroll through the worlds of animals and men: A picture book of invisible worlds, Semiotica 89 (4) (1992) 319-391 (1992). doi:10.1515/semi.1992.89.4.319

[28] N. Wiener, Cybernetics, or Control and Communication in the Animal and the Machine, The Technology Press, New York, 1948 (1948).

[29] C. Maureira, J. C. Letelier, O. Alvarez, R. Delgado, C. Vergara, Copper enhances cellular and network excitabilities, and improves temporal processing in the rat hippocampus, European Journal of Neuroscience 42 (12) (2015). doi:10.1111/ejn.13104

[30] H. R. Maturana, Autopoiesis, Structural Coupling and Cognition : A history of these and other notions in the biology of cognition, Cybernetics \& Human Knowing 9 (3) (2002) 5-34 (2002). doi:10.1111/j.1471-1842.2008.00767.x

URL http://www . ingentaconnect . com/content/imp/chk/2002/00000009/f0020003/121 
[31] R. Rosen, A relational theory of biological systems, The Bulletin of Mathematical Biophysics 20 (3) (1958) 245-260 (sep 1958). doi:10.1007/BF02478302.

URL http://link.springer.com/10.1007/BF02478302http://link.springer.com/10. 1007/BF02476354

[32] T. Nomura, Category Theoretical Distinction Between Autopoiesis and (M,R) Systems, in: Advances in Artificial Life, Springer Berlin Heidelberg, Berlin, Heidelberg, 2007, pp. 465-474 (2007). doi:10.1007/978-3-540-74913-4_47.

URL http://link.springer.com/10.1007/978-3-540-74913-4\{_\}47

[33] G. Spencer-Brown, Laws of form, Julian Press, 1972 (1972).

URL https://books.google.cl/books?id=JbtKAAAAMAAJ

[34] F. J. Varela, Principles of Biological Autonomy, Developments in Marine Biology, North Holland, 1979 (1979).

URL https://books .google.cl/books?id=kdyGQgAACAAJ

[35] C. J. Scriba, P. Schreiber, 5000 Years of Geometry: Mathematics in History and Culture, Birkhäuser, 2015 (2015).

[36] E. Stein, The History of Theoretical, Material and Computational Mechanics - Mathematics Meets Mechanics and Engineering, no. January 2014, Springer-Verlag Berlin Heidelberg, 2013 (2013). doi:10.1007/978-3-642-39905-3.

URL https://books . google.com/books?id=qja8BAAAQBAJ $\{\&\}$ pgis=1

[37] G. Rudolph, M. Schmidt, Differential Geometry and Mathematical Physics: Part I. Manifolds, Lie Groups and Hamiltonian Systems, Vol. 1011, 2017 (2017). arXiv:arXiv:1011.1669v3, doi:10.1007/978-94-007-5345-7.

URL http://link.springer.com/10.1007/978-94-007-5345-7

[38] G. Rudolph, M. Schmidt, Differential Geometry and Mathematical Physics. Part II. Fibre Bundles, Topology and Gauge Fields, 2017 (2017). arXiv:arXiv:1011.1669v3, doi:10. 1007/978-94-007-5345-7.

URL http://link.springer.com/10.1007/978-94-007-5345-7 
[39] T. Lee, M. Leok, N. H. McClamroch, Global Formulations of Lagrangian and Hamiltonian Dynamics on Manifolds, Interaction of Mechanics and Mathematics, Springer International Publishing, Cham, 2018 (2018). doi:10.1007/978-3-319-56953-6.

URL http://link.springer.com/10.1007/978-3-319-56953-6

[40] T. Lee, M. Leok, N. H. McClamroch, Lagrangian and Hamiltonian Dynamics on Manifolds, 2018, pp. 347-398 (2018). doi:10.1007/978-3-319-56953-6_8

URL http://link.springer.com/10.1007/978-3-319-56953-6\{_\}8

[41] S. Wiggins, Introduction to applied nonlinear dynamical systems and chaos, second ed Edition, Springer Berlin Heidelberg, 2003 (2003).

[42] I. N. Bronshtein, K. A. Semendyayev, G. Musiol, H. Muhlig, Handbook of Mathematics, sixth edit Edition, Vol. 18, Springer, Berlin, Heidelberg, 2015 (2015). arXiv:arXiv:1011.1669v3 doi:10.1007/978-3-662-46221-8.

URL http://link.springer.com/10.1007/978-3-662-46221-8

[43] C. Skiadas, The Foundations of Chaos Revisited: From Poincaré to Recent Advancements 2016 (2016). doi:10.1007/978-3-319-29701-9

URL http://link. springer.com/10.1007/978-3-319-29701-9

[44] A. Deriglazov, Classical Mechanics, 2017 (2017). arXiv:arXiv:1011.1669v3, doi:10.1007/ 978-3-319-44147-4.

URL http://link. springer.com/10.1007/978-3-319-44147-4

[45] V.-T. Pham, S. Vaidyanathan, C. Volos, T. Kapitaniak, Nonlinear Dynamical Systems with Self-Excited and Hidden Attractors, Vol. 133 of Studies in Systems, Decision and Control, Springer International Publishing, Cham, 2018 (2018). doi:10.1007/978-3-319-71243-7. URL http://link.springer.com/10.1007/978-3-319-71243-7

[46] A. C. Reinol, M. Messias, Periodic orbits, invariant tori and chaotic behavior in certain nonequilibrium quadratic three-dimensional differential systems, in: Nonlinear Dynamical Systems with Self-Excited and Hidden Attractors, Vol. 133, 2018, pp. 299-326 (2018). doi: 10.1007/978-3-319-71243-7_13. 
[47] V. G. Ivancevic, T. T. Ivancevic, Complex Nonlinearity: Chaos, Phase Transitions, Topology Change and Path Integrals, Springer, 2008 (2008). doi:10.1007/978-3-642-33902-8.

[48] V. G. Ivancevic, T. T. Ivancevic, Natural Biodynamics, World Scientific, 2005 (2005). URL https://books.google.cl/books?id=udxgDQAAQBAJ

[49] V. G. Ivancevic, T. T. Ivancevic, Quantum Neural Computation, 2010 (2010). doi:10.1007/ 978-90-481-3350-5.

URL http://link.springer.com/10.1007/978-90-481-3350-5

[50] A. Weinstein, Symplectic geometry, Bulletin of the American Mathematical Society 5 (1) (1981) 1-13 (jul 1981). arXiv:0505366, doi:10.1090/S0273-0979-1981-14911-9.

[51] Á. Pelayo, S. V. Ngoc, Symplectic Theory, Bulletin of the American Mathematical Society 48 (3) (2011) 409-455 (2011). doi:10.1090/S0273-0979-2011-01338-6.

[52] Á. Pelayo, Hamiltonian and symplectic symmetries: An introduction, Bulletin of the American Mathematical Society 54 (3) (2017) 383-436 (mar 2017). arXiv:1610.09718, doi: $10.1090 / \mathrm{bull} / 1572$

[53] A. Knauf, Mathematical Physics: Classical Mechanics, 2018 (2018).

[54] J. Jost, Mathematical concepts, Springer International Publishing, 2015 (2015).

[55] V. I. Arnold, V. V. Kozlov, A. I. Neishtadt, Mathematical Aspects of Classical and Celestial Mechanics, 3rd Edition, no. v. 3 in Encyclopaedia of mathematical sciences, Springer, Berlin ; New York, 2006 (2006). arXiv:0402023, doi:10.1007/978-3-540-48926-9. URL http://books . google.com.hk/books?id=PROuMQEACAAJ

[56] L. Ji, A. Papadopoulos, Sophus Lie and Felix Klein : the Erlangen program and its impact in mathematics and physics, European Mathematical Society, 2015 (2015).

[57] J. Baez, J. D. Biamonte, Quantum Techniques in Stochastic Mechanics, WORLD SCIENTIFIC, 2018 (2018). doi:10.1142/10623

URL https://www .worldscientific.com/doi/abs/10.1142/10623

[58] C. Skokos, G. A. Gottwald, J. Laskar, Chaos Detection and Predictability, 2016 (2016). doi:10.1007/978-3-319-03771-4. 
[59] C. Luo, S. Soskin, R. Mannella, O. Yevtushenko, I. Khovanov, P. McClintock, X. Leoncini, S. Prants, T. Fromhold, A. Krokhin, S. Bujkiewicz, P. Wilkinson, D. Fowler, A. Patane, L. Eaves, D. Hardwick, A. Balanov, M. Greenaway, A. Henning, A. Virovlyansky, Hamiltonian Chaos Beyond the KAM Theory, Vol. 0 of Nonlinear Physical Science, Springer Berlin Heidelberg, Berlin, Heidelberg, 2010 (2010). doi:10.1007/978-3-642-12718-2

URL http://link . springer.com/10.1007/978-3-642-12718-2

[60] M. Toda, Geometric Structures of Phase Space in Multidimensional Chaos: Applications to Chemical Reaction Dynamics in Complex Systems, no. pt. 2 in Advances in chemical physics, Wiley, 2005 (2005).

URL https://books.google.cl/books?id=QyFEAQAAIAAJ

[61] E. Schrödinger, What is Life?: The Physical Aspect of the Living Cell, The University Press, 1962 (1962).

URL https://books.google.cl/books?id=jmULAQAAIAAJ

[62] J. C. Baez, B. Fong, Quantum techniques for studying equilibrium in reaction networks, Journal of Complex Networks 3 (1) (2015) 22-34 (2015). arXiv:1305.4988, doi:10.1093/ comnet/cnu013.

URL https : //academic.oup.com/comnet/article-abstract/3/1/22/490572

[63] A. van der Schaft, D. Jeltsema, Port-Hamiltonian Systems Theory: An Introductory Overview, Now Publishers Incorporated, 2014 (2014). doi:10.1561/2600000002.

URL http://dx.doi .org/10.1561/2600000002

[64] C. Maureira, Quantum Bio-Systems from Biological Organization Principles. Organizational Principles Underpinning Quantum Phenomenology in Biology: Information, Computing, Cognition and Decision Making, in preparation (2019).

[65] M. F. Weber, E. Frey, Master equations and the theory of stochastic path integrals Reports on Progress in Physics 80 (4) (2017) 46601 (2017). arXiv:1609.02849 doi:10.1088/1361-6633/aa5ae2.

URL http://stacks.iop.org/0034-4885/80/i=4/a=046601?key=crossref . 02abaf744081951aaaca3bafec0e1284 
[66] R. P. Feynman, A. R. Hibbs, D. F. Styer, Quantum Mechanics and Path Integrals, Dover Books on Physics, Dover Publications, 2010 (2010).

URL https://books .google.cl/books?id=JkMuDAAAQBAJ

[67] H. Kleinert, Path Integrals in Quantum Mechanics, Statistics, and Polymer Physics, 2nd Edition, WORLD SCIENTIFIC, 1995 (1995). doi:10.1142/2092

URL https://www . worldscientific.com/doi/abs/10.1142/2092

[68] S. Lloyd, O. Dreyer, The universal path integral, Quantum Information Processing 15 (2) (2016) 959-967 (2016). arXiv:1302.2850, doi:10.1007/s11128-015-1178-7.

[69] C. Kuehn, Multiple Time Scale Dynamics, Vol. 191 of Applied Mathematical Sciences, Springer International Publishing, Cham, 2015 (2015). doi:10.1007/978-3-319-12316-5. URL http://link.springer.com/10.1007/978-3-319-12316-5

[70] V. I. Arnold, A. B. Givental, B. Khesin, J. E. Marsden, A. N. Varchenko, V. A. Vassiliev, O. Viro, V. Zakalyukin, Vladimir I. Arnold - Collected Works: Representations of Functions, Celestial Mechanics, and KAM Theory 1957-1965, Vladimir I. Arnold - Collected Works, Springer Berlin Heidelberg, 2009 (2009).

URL https://books . google.cl/books?id=Noj8\{_\}KqLEYkC

[71] B. V. Chirikov, A universal instability of many-dimensional oscillator systems, Physics Reports 52 (5) (1979) 263-379 (1979). doi:10.1016/0370-1573(79)90023-1.

[72] C. Brentari, Jakob von Uexküll: The Discovery of the Umwelt between Biosemiotics and Theoretical Biology, Springer Science+Business Media, 2015 (2015).

[73] J. Hoffmeyer, Biosemiotics: An Examination Into the Signs of Life and the Life of Signs, Approaches to postmodernity, University of Scranton Press, 2008 (2008).

URL https://books . google.cl/books?id=R-7aAAAAMAAJ

[74] M. Feinberg, Complex balancing in general kinetic systems, Archive for Rational Mechanics and Analysis 49 (3) (1972) 187-194 (jan 1972). doi:10.1007/BF00255665

URL http://link.springer.com/10.1007/BF00255665

[75] M. Feinberg, Chemical reaction network structure and the stability of complex isothermal reactors-I. The deficiency zero and deficiency one theorems, Chemical Engineering Science 
42 (10) (1987) 2229-2268 (jan 1987). doi:10.1016/0009-2509(87)80099-4.

URL https://www.sciencedirect.com/science/article/pii/0009250987800994? via $\{\%\} 3$ Dihub

[76] F. Horn, Necessary and sufficient conditions for complex balancing in chemical kinetics, Archive for Rational Mechanics and Analysis 49 (3) (1972) 172-186 (jan 1972). doi: 10.1007/BF00255664.

URL http://link. springer . com/10.1007/BF00255664

[77] A. van der Schaft, S. Rao, B. Jayawardhana, Balanced chemical reaction networks governed by general kinetics, Proceedings fo 20th Mathematical Theory of Networks and Systems 73 (2) (2012) 1-4 (2012).

[78] A. van der Schaft, S. Rao, B. Jayawardhana, On the Mathematical Structure of Balanced Chemical Reaction Networks Governed by Mass Action Kinetics (2011). arXiv:1110.6078, doi:10.1137/11085431X,

URL http://arxiv.org/abs/1110.6078

[79] A. van der Schaft, A. V. D. Schaft, Chemical reaction networks : a system-theorist's point of view (June) (2009) 1-43 (2009).

[80] H. Maturana, J. Mpodozis, The origin of species by means of natural drift (2000). doi:10.4067/S0716-078X2000000200005.

URL http://www.scielo.cl/scielo.php?script=sci\{_\}arttext $\{\&\}$ pid= S0716-078X2000000200005\{\&\}lng=en $\{\&\}$ nrm=iso $\{\&\}$ tlng=en

[81] B. Hasselblatt, H. Broer, F. Takens, Handbook of dynamical systems, North-Holland/Elsevier, Amsterdam ; Boston, 2010 (2010).

URL http://sci-hub.cc/https://books.google.com/books?hl=en $\{\&\} l r=\{\&\}$ id= mJYw3u2HniUC $\{\&\} \circ i=f n d\{\&\}$ pg=PP1 $\{\&\}$ dq=Handbook+of+Dynamical+Systems , +Volume+ $3\{\&\}$ ots $=1 \times 1 \mathrm{~m} 2 \mathrm{ht} 6 \mathrm{Cu}\{\&\}$ sig $=623 \mathrm{pDYsQJOG}\left\{\left\{_{-}\right\} \mathrm{R}\left\{\_\right\} \mathrm{Gj} 9\left\{_{-}\right\} \mathrm{ZwA} 23 \mathrm{NaUE}\right.$

[82] E. E. Charpentier, A. E. Lesne, N. E. Nikolski, Kolmogorov's Heritage in Mathematics, Vol. 53, Springer, Berlin ; New York, 2007 (2007). arXiv:arXiv:1011.1669v3, doi:10. 1007/978-3-540-36351-4. 
[83] J. D. Meiss, Visual explorations of dynamics: The standard map, Pramana - Journal of Physics 70 (6) (2008) 965-988 (2008). arXiv:0801.0883, doi:10.1007/s12043-008-0103-3

[84] H. Kantz, P. Grassberger, Internal Arnold diffusion and chaos thresholds in coupled symplectic maps, Journal of Physics A: Mathematical and General 21 (3) (1988) L127-L133 (feb 1988). doi:10.1088/0305-4470/21/3/003

URL https://doi.org/10.1088\{\%\}2F0305-4470\{\%\}2F21\{\%\}2F3\{\%\}2F003

[85] A. J. Lichtenberg, M. A. Lieberman, Regular and Chaotic Dynamics, Applied Mathematical Sciences, Springer New York, 2013 (2013).

URL https://books .google.cl/books?id=pR3aBwAAQBAJ

[86] D. F. D. F. Anderson, G. Craciun, T. G. T. G. Kurtz, Product-form stationary distributions for deficiency zero chemical reaction networks, arXiv : 0803.3042v4 (2010) 1-23 (2010). arXiv:arXiv:0803.3042v4, doi:10.1007/s11538-010-9517-4, URL http://www.springerlink.com/index/nh2t8862q72q4481.pdf

[87] L. Aitchison, M. Lengyel, The Hamiltonian Brain: Efficient Probabilistic Inference with Excitatory-Inhibitory Neural Circuit Dynamics, PLOS Computational Biology 12 (12) (2016) e1005186 (dec 2016). arXiv:1407.0973, doi:10.1371/journal.pcbi.1005186.

URL http://arxiv.org/abs/1407.0973https://dx.plos.org/10.1371/journal.pcbi. 1005186

[88] K. Friston, J. Kilner, L. Harrison, A free energy principle for the brain, Journal of Physiology Paris 100 (1-3) (2006) 70-87 (2006). arXiv:arXiv:1401.4122v2, doi:10.1016/j. jphysparis.2006.10.001.

[89] K. Friston, A theory of cortical responses., Philosophical transactions of the Royal Society of London. Series B, Biological sciences 360 (1456) (2005) 815-836 (2005). doi:10.1098/rstb.2005.1622

URL http://www.pubmedcentral.nih.gov/articlerender.fcgi?artid= $1569488\{\&\}$ tool=pmcentrez $\{\&\}$ rendertype=abstract

[90] K. Friston, Learning and inference in the brain, Neural Networks 16 (9) (2003) 1325-1352 (2003). doi:10.1016/j.neunet.2003.06.005 
[91] M. J. D. Ramstead, P. B. Badcock, K. J. Friston, M. J. Désormeau Ramstead, P. B. Badcock, K. J. Friston, D. M. J. Ramstead, P. B. Badcock, K. J. Friston, Answering Schrödinger's question: A free-energy formulation, Physics of Life Reviews 24 (2018) 1-16 (2018). doi:10.1016/j.plrev.2017.09.001.

URL http://linkinghub.elsevier.com/retrieve/pii/S1571064517301409http: //dx.doi.org/10.1016/j.plrev.2017.09.001

[92] D. Rudrauf, D. Bennequin, I. Granic, G. Landini, K. Friston, K. Williford, A mathematical model of embodied consciousness, Journal of Theoretical Biology (2017). doi:10.1016/j . jtbi.2017.05.032. URL http://dx.doi.org/10.1016/j.jtbi.2017.05.032

[93] K. J. Friston, K. H. Preller, C. Mathys, H. Cagnan, J. Heinzle, A. Razi, P. Zeidman, NeuroImage Dynamic causal modelling revisited, NeuroImage (February) (2017) 0-1 (2017). doi:10.1016/j.neuroimage.2017.02.045

URL http://dx.doi.org/10.1016/j.neuroimage.2017.02.045

[94] Y. M. Yufik, K. Friston, Life and Understanding: The Origins of "Understanding" in SelfOrganizing Nervous Systems, Frontiers in Systems Neuroscience 10 (December) (2016) 98 (2016). doi:10.3389/fnsys.2016.00098.

[95] C. L. Buckley, C. S. Kim, S. McGregor, A. K. Seth, The free energy principle for action and perception: A mathematical review (dec 2017). arXiv:1705.09156, doi:10.1016/j.jmp. 2017.09 .004

[96] B. Sengupta, A. Tozzi, G. K. Cooray, P. K. Douglas, K. J. Friston, Towards a Neuronal Gauge Theory, PLoS Biology 14 (3) (2016) e1002400 (mar 2016). doi:10.1371/journal. pbio. 1002400

[97] M. Allen, K. J. Friston, From cognitivism to autopoiesis: towards a computational framework for the embodied mind, Synthese 195 (6) (2018) 2459-2482 (jun 2018). doi:10.1007/ s11229-016-1288-5.

URL https://pubmed.ncbi.nlm.nih.gov/29887647/

[98] E. T. Jaynes, Information theory and statistical mechanics, Physical Review 106 (4) (1957) 620-630 (1957). arXiv:arXiv:1011.1669v3, doi:10.1103/PhysRev.106.620. 
[99] C. E. Shannon, A mathematical theory of communication, The Bell System Technical Journal 27 (July 1928) (1948) 379-423 (1948). arXiv:9411012, doi:10.1145/584091.584093. URL http://cm.bell-labs.com/cm/ms/what/shannonday/shannon1948.pdf

[100] M. S. Dodd, D. Papineau, T. Grenne, J. F. Slack, M. Rittner, F. Pirajno, J. O'Neil, C. T. S. Little, Evidence for early life in Earth's oldest hydrothermal vent precipitates, Nature 543 (7643) (2017) 60-64 (mar 2017). doi:10.1038/nature21377.

URL http://www .nature.com/doifinder/10.1038/nature21377

[101] E. A. Bell, P. Boehnke, T. M. Harrison, W. L. Mao, Potentially biogenic carbon preserved in a 4.1 billion-year-old zircon, Proceedings of the National Academy of Sciences 112 (47) (2015) 14518-14521 (2015). arXiv:arXiv:1408.1149, doi:10.1073/pnas.1517557112

URL http://www.pnas.org/lookup/doi/10.1073/pnas.1517557112

[102] M. L. Cárdenas, Life before LUCA, Journal of Theoretical Biology 434 (2017) 68-74 (2017). doi:10.1016/j.jtbi.2017.05.023.

[103] H. Maturana, F. Varela, Autopoiesis and Cognition: The Realization of the Living, 1991 (1991).

URL http://www . amazon.com/Autopoiesis-Cognition-Realization-Studies-Philosophy/ $\mathrm{dp} / 9027710163$

[104] C. Mora, D. P. Tittensor, S. Adl, A. G. B. Simpson, B. Worm, How Many Species Are There on Earth and in the Ocean?, PLoS Biology 9 (8) (2011) e1001127 (aug 2011). doi: 10.1371/journal.pbio.1001127. URL http://dx.plos.org/10.1371/journal.pbio.1001127

[105] J. von Uexküll, Theoretical Biology, Harcourt, Brace \& Co., New York, 1926 (1926).

[106] F. Capra, P. L. Luisi, The systems view of life: a unifying vision, Vol. 11, 2014 (2014). doi:10.1017/CB09780511895555. 